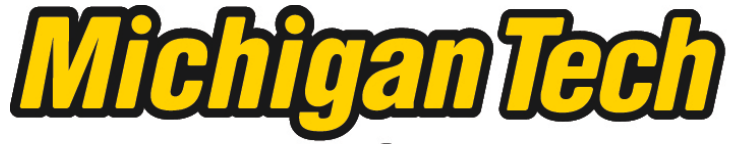 \\ Michigan Technological University Create the Future Digital Commons @ Michigan Tech
}

Dissertations, Master's Theses and Master's Reports - Open

Dissertations, Master's Theses and Master's

Reports

2014

\section{MILITARY VEHICLE OPTIMIZATION AND CONTROL}

Denise M. Rizzo

Michigan Technological University

Follow this and additional works at: https://digitalcommons.mtu.edu/etds

Part of the Mechanical Engineering Commons

Copyright 2014 Denise M. Rizzo

\section{Recommended Citation}

Rizzo, Denise M., "MILITARY VEHICLE OPTIMIZATION AND CONTROL", Dissertation, Michigan Technological University, 2014.

https://doi.org/10.37099/mtu.dc.etds/863

Follow this and additional works at: https://digitalcommons.mtu.edu/etds

8 Part of the Mechanical Engineering Commons 


\section{MILITARY VEHICLE OPTIMIZATION AND CONTROL}

By

Denise M. Rizzo

\section{A DISSERTATION}

Submitted in partial fulfillment of the requirements for the degree of DOCTOR OF PHILOSOPHY

In Mechanical Engineering - Engineering Mechanics

\section{MICHIGAN TECHNOLOGICAL UNIVERSITY \\ 2014}

(C) 2014 Denise M. Rizzo 

This dissertation has been approved in partial fulfillment of the requirements for the Degree of DOCTOR OF PHILOSOPHY in Mechanical Engineering - Engineering Mechanics.

Department of Mechanical Engineering - Engineering Mechanics

Dissertation Advisor: Dr. Gordon G. Parker

Committee Member: Dr. Wayne W. Weaver

Committee Member: Dr. John E. Beard

Committee Member: Dr. Alexander Reid

Department Chair: Dr. William W. Predebon 



\section{Contents}

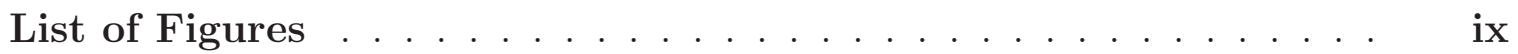

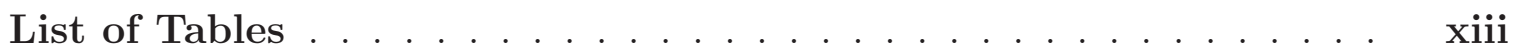

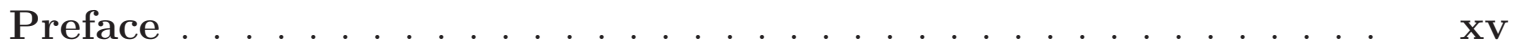

Acknowledgments ................. xvii

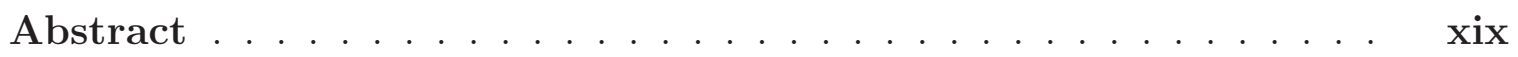

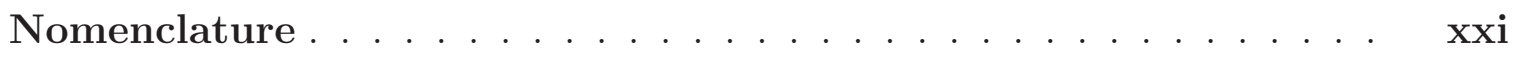

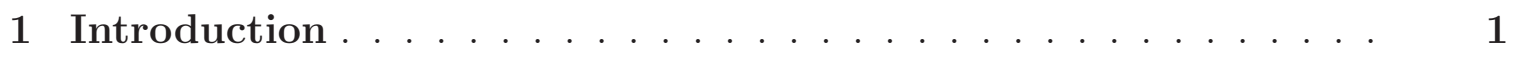

1.1 Motivation . . . . . . . . . . . . . . . . . . . 2

1.2 Research Background . . . . . . . . . . . . . . . . 5

1.2.1 Military Application of Hybrid Systems . . . . . . . . . . 6

1.2.1.1 Challenges . . . . . . . . . . . . . 6

1.2.1.2 Opportunity . . . . . . . . . . . . 6

1.2.2 Vehicle and Powertrain Overview . . . . . . . . . 7

1.2.2.1 Vehicles ................. 8

1.2.2.2 Parallel Powertrain . . . . . . . . . . . . . 8

1.2.2.3 Series Powertrain . . . . . . . . . . . . . 10

1.2 .3 Duty Cycle Overview . . . . . . . . . . . . . . . . . . 10

1.2.4 Documented Fuel Economy Improvements . . . . . . . . . 12

1.2.4.1 Parallel Powertrain . . . . . . . . . . . . . . 12

1.2.4.2 Series Powertrain . . . . . . . . . . . . . . 13

1.2.4.3 Drive Cycle Impact . . . . . . . . . . . . . . . 13

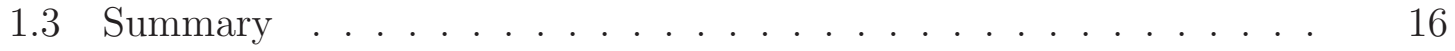




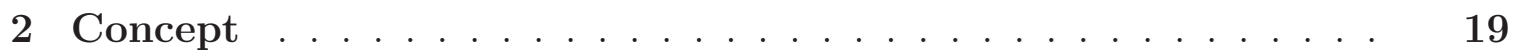

2.1 Research Objective and Scope . . . . . . . . . . . . . . . . . . 19

2.2 Microgrid Introduction . . . . . . . . . . . . . . . . . 20

3 Duty Cycles and Their Adaptation to Military Hybrid Vehicles 23

3.1 Propulsion Cycle . . . . . . . . . . . . . . . . . . . . . . 23

3.2 Electrical Cycle . . . . . . . . . . . . . . . . . . . . . . . 24

3.3 Stationary Microgrid . . . . . . . . . . . . . . . . . 26

4 Vehicle Model . . . . . . . . . . . . . . . . . . . . . . . . . . . . . 29

4.1 Overview . . . . . . . . . . . . . . . . . . . . . . 30

4.2 Internal Combustion Engine . . . . . . . . . . . . . . . . . 33

4.3 Electric Machine Performance . . . . . . . . . . . . . . . . . 35

4.4 Vehicle Model Implementation . . . . . . . . . . . . . . . . . . . 37

5 Basis Function $S O C$ Optimization $\ldots \ldots \ldots$

5.1 Fuel optimal $S O C$ problem definition . . . . . . . . . . . . . . 41

5.2 Step 1: Drive Cycle Decomposition . . . . . . . . . . . . 44

5.3 Step 2: SOC Optimization . . . . . . . . . . . . . . 47

5.4 Results . . . . . . . . . . . . . . . . . . . . . . 48

5.5 Discussion . . . . . . . . . . . . . . . . . . . . . . 50

5.6 Summary . . . . . . . . . . . . . . . . . . . . . . . . 52

6 Multiple Input Optimization . . . . . . . . . . . . . . . . 53

6.1 Problem Formulation . . . . . . . . . . . . . . . . . . . 54

6.2 Constraints . . . . . . . . . . . . . . . . . . . . . 59

6.3 Numerical Integration Considerations . . . . . . . . . . . . . 60

6.4 Final Description of Numerical Optimization Problem . . . . . . . 62

7 Results ........................... . . . 65

7.1 Power System Control . . . . . . . . . . . . . . . . . 65

7.1.1 Problem Statement . . . . . . . . . . . . . . . . . 65

7.1.2 Closed Loop Control . . . . . . . . . . . . . . . . . . . 67

7.1.3 Controller Comparison Results . . . . . . . . . . . . 70

7.1.3.1 Stationary Grid Requirement . . . . . . . . . . 72

7.1.3.2 Electrical Cycle Parametric Study _. . . . . . . 78 
7.2 Design Optimization . . . . . . . . . . . . . . . . 79

7.2.1 Problem statement . . . . . . . . . . . . . . . . . 80

7.2.2 System Component Design Results . . . . . . . . . . . . . . 82

7.3 Summary . . . . . . . . . . . . . . . . . . 87

8 Summary and Conclusions . . . . . . . . . . . . . . . . . . 89

8.1 Summary . . . . . . . . . . . . . . . . 89

8.2 Conclusions . . . . . . . . . . . . . . . . . . . 92

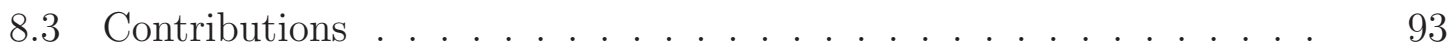

8.4 Future Work . . . . . . . . . . . . . . . . . 93

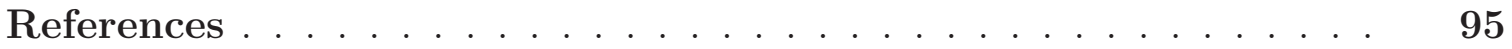

A Code ................................ 107

B Simplified Vehicle Model Supporting Equations . . . . . . . . . 115

C Mupad Code for Linearization . . . . . . . . . . . . . . . 117

D Supporting Figures for Control . . . . . . . . . . . . . 121

E Supporting Figures for Design Optimization . . . . . . . . . . 127

F Letters of Permission . . . . . . . . . . . . . . . . . 131 



\section{List of Figures}

1.1 Class III HMMWV . . . . . . . . . . . . . . . . . . . 8

1.2 Class VI - VII FMTV . . . . . . . . . . . . . . . . . 9

1.3 Class VIII HEMMTT . . . . . . . . . . . . . . . . . . . . . . . 9

1.4 Time dependent speed profiles . . . . . . . . . . . . . . . 11

1.5 Distance dependent grade profiles . . . . . . . . . . . . . . 11

1.6 Cycle vs. fuel economy improvement for the HMMWV . . . . . . . 14

1.7 Cycle vs. fuel economy improvement for the class VI vehicle . . . . 15

1.8 Cycle vs. fuel economy improvement for the class VII \& VIII vehicle 15

2.1 Overview of a generic stationary microgrid . . . . . . . . . 21

2.2 Overview of a vehicle microgrid . . . . . . . . . . . . 22

2.3 Overview of a vehicle integrated into a stationary microgrid . . . 22

3.1 Cycle vs. fuel economy improvement for the HMMWV (originally shown in Chapter 1) . . . . . . . . . . . . . . . 24

3.2 Propulsion duty cycle . . . . . . . . . . . . . . . . . . 25

3.3 Electrical duty cycles . . . . . . . . . . . . . . . . . . . . 26

4.1 Power split overview . . . . . . . . . . . . . . . . 30

4.2 Engine torque curve . . . . . . . . . . . . . . . . . 34

4.3 Engine fuel surface $(g / k W h) \ldots \ldots \ldots \ldots \ldots$

4.4 Motor torque curve . . . . . . . . . . . . . . . 35

4.5 Generator torque curve . . . . . . . . . . . . . 36

4.6 Motor efficiency surface $(\%) \ldots \ldots \ldots \ldots \ldots$

4.7 Generator efficiency surface $(\%) \ldots \ldots \ldots \ldots \ldots$

5.1 Two step optimization overview . . . . . . . . . . . . . . 40

5.2 Map for relating engine speed and engine torque to fuel consumption. 41

5.3 Military duty cycle - urban assault . . . . . . . . . . . . 44 
5.4 Measured $\left(v_{m}\right)$ vs. approximate $(\tilde{v})$ vehicle Speed . . . . . . . . . 47

$5.5 S O C$ comparison . . . . . . . . . . . . . . . 50

$5.6 S O C$ comparison with simplified model . . . . . . . . . . . . 51

6.1 Map for relating engine speed $\left(\omega_{e}\right)$ and torque $\left(T_{e}\right)$ to fuel consump-

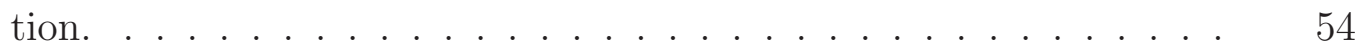

6.2 Propulsion duty cycle (originally shown in Chapter3) . . . . . . . 57

6.3 Calculation order . . . . . . . . . . . . . . . . 59

6.4 Urban assault cycle . . . . . . . . . . . . . . . . . . . . 60

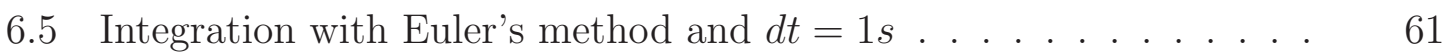

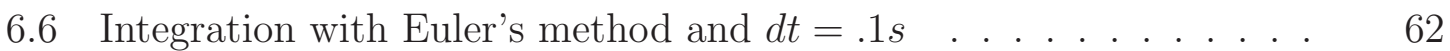

7.1 Propulsion duty cycle (originally shown in Chapter3) . . . . . . 66

7.2 Electrical duty cycle (originally shown in Chapter3) . . . . . . . 66

7.3 Closed loop control . . . . . . . . . . . . . . . . 67

7.4 Closed loop eigenvalues . . . . . . . . . . . . . . . . . 69

7.5 Engine torque trajectories . . . . . . . . . . . . . 70

7.6 Generator torque trajectories . . . . . . . . . . . . . 71

7.7 Instantaneous fuel used . . . . . . . . . . . . . . . . . . . . . . 71

7.8 Battery state of charge . . . . . . . . . . . . . . . . 72

7.9 Motor speed . . . . . . . . . . . . . . . . 73

7.10 Constraints for both control systems . . . . . . . . . . . . 73

7.11 Cost of each case . . . . . . . . . . . . . . . . . . 74

7.12 Engine torque trajectories with new case . . . . . . . . . . 75

7.13 Generator torque trajectories with new case . . . . . . . . . 75

7.14 Battery state of charge trajectories with new case . . . . . . . 76

7.15 Instantaneous fuel used for each case . . . . . . . . . . . . . 76

7.16 Motor speed for each case . . . . . . . . . . . . . 77

7.17 Constraints for each case . . . . . . . . . . . . . . . . 77

7.18 Electrical duty cycle test definition . . . . . . . . . . . . . 78

7.19 Cost of comparison for electrical duty cycle sensitivity analysis . . . 79

7.20 Battery and fuel cost for different electrical duty cycles . . . . . . . 82

7.21 Engine torque trajectories for each electrical duty cycle . . . . . . . 83

7.22 Generator torque trajectories for each electrical duty cycle . . . . . 83

7.23 Brake force torque trajectories for each electrical duty cycle . . . . . 84 
7.24 Engine speed for each electrical duty cycle . . . . . . . . . . . . 84

7.25 Battery state of charge for each electrical duty cycle . . . . . . . 85

7.26 Fuel used for each electrical duty cycle . . . . . . . . . . . 86

7.27 Motor speed for each electrical duty cycle . . . . . . . . . . . . 86

7.28 Constraints for each electrical duty cycle . . . . . . . . . . . 87

D.1 Brake force torque trajectories . . . . . . . . . . . . . 121

D.2 Motor torque trajectory . . . . . . . . . . . . . . . 122

D.3 Engine speed acceleration . . . . . . . . . . . . . . . 122

D.4 Generator speed . . . . . . . . . . . . . . . 123

D.5 Brake force torque trajectory for all three cases . . . . . . . . 123

D.6 Motor torque trajectory for all three cases . . . . . . . . . . . . . 124

D.7 Engine speed for all three cases . . . . . . . . . . . . . . . 124

D.8 Generator speed for all three cases . . . . . . . . . . . 125

D.9 Motor speed for all three cases . . . . . . . . . . . . 125

E.1 Motor torque trajectories for different electrical duty cycles . . . . . 128

E.2 Engine speed acceleration for different electrical duty cycles . . . . 128

E.3 Generator speed for different electrical duty cycles . . . . . . . . . . 129 



\section{List of Tables}

1.1 Fuel savings for class III and IV trucks predicted by the study of reference $[1] . \ldots \ldots \ldots \ldots 7 \ldots$. . . . . . . . . . . . . . . . . . 7

4.1 Vehicle parameters . . . . . . . . . . . . . . . . 38

5.1 Rule based engine speed $\left(\omega_{e}\right)$ control . . . . . . . . . . . 43

5.2 Cumulative residual error .................. . . . 45 



\section{Preface}

\section{Copyright Permission}

Chapter 1 is reprinted with permission from "Current state of military hybrid vehicle development," Int. J. Electric and Hybrid Vehicles, Vol. 3, No. 4, pp.369 - 387 (C) Inderscience Publishers. Further use or distribution is not permitted without permission from Inderscience. I performed the literature search, compiled the data and wrote paper. My co-author proofread and peer reviewed the paper for originality.

Chapter 5 is reprinted with permission from "Determining optimal state of charge for a military vehicle microgrid," Int. J. Powertrains, Vol. 3, No. 3, pp.303 - 318 (C) Inderscience Publishers. Further use or distribution is not permitted without permission from Inderscience. I wrote the code, ran the simulations, compiled the results and wrote paper. My co-author proofread and peer reviewed the paper for originality.

The letter of permission can be found in Appendix F.

\section{Distribution Statement}

Unclassified. Distribution Statement A. Approved for public release.

\section{Disclaimer}

Reference herein to any specific commercial company, product, process, or service by trade name, trademark, manufacturer, or otherwise, does not necessarily constitute or imply its endorsement, recommendation, or favoring by the United States Government or the Department of the Army (DoA). The opinions of the authors expressed herein do not necessarily state or reflect those of the United States Government or the DoA, and shall not be used for advertising or product endorsement purposes. 



\section{Acknowledgments}

First, I would like to acknowledge the U.S. Army TARDEC for providing funding for this work. More specifically, I would like to thank Mr. Michael K. Pozolo, my team leader, for the unwavering support over the last five years. Without his professional support and encouragement, I would have never succeeded on this journey.

Next, I have to thank Dr. Gordon G. Parker, my advisor, who pulled, pushed and stood by me at all of the important moments. His enthusiasm and intelligence constantly made me want to learn more and do more. Thanks for being an advisor, mentor and friend.

"Hard work pays off!" How many times did my parents tell me that? Next, I have to thank my parents, Mary Ann and Harry Rizzo, for teaching me a boundless work ethic and anything is possible, if you are willing to work for it. I would have never gone down this path if it weren't for them. So, thank you.

Last, but certainly not least, I would like to thank my family. Mr. Andrew L. Wiegand, my husband, your undying support for my winding journey is the one of the reasons why I am so incredibly lucky. You are the foundation of our family and this would have been impossible without you. Ms. Amelia A. Wiegand, my daughter, you are my inspiration and my drive to continually be a better person. And, Rusty Rizzo, my loyal dog, you are my most devoted friend in the world and life would not be the same without you. 



\section{Abstract}

It is remarkable that there are no deployed military hybrid vehicles since battlefield fuel is approximately 100 times the cost of civilian fuel. In the commercial marketplace, where fuel prices are much lower, electric hybrid vehicles have become increasingly common due to their increased fuel efficiency and the associated operating cost benefit. An absence of military hybrid vehicles is not due to a lack of investment in research and development, but rather because applying hybrid vehicle architectures to a military application has unique challenges. These challenges include inconsistent duty cycles for propulsion requirements and the absence of methods to look at vehicle energy in a holistic sense. This dissertation provides a remedy to these challenges by presenting a method to quantify the benefits of a military hybrid vehicle by regarding that vehicle as a microgrid. This innovative concept allowed for the creation of an expandable multiple input numerical optimization method that was implemented for both real-time control and system design optimization. An example of each of these implementations was presented. Optimization in the loop using this new method was compared to a traditional closed loop control system and proved to be more fuel efficient. System design optimization using this method successfully illustrated battery size optimization by iterating through various electric duty cycles. By utilizing this new multiple input numerical optimization method, a holistic view of duty cycle synthesis, vehicle energy use, and vehicle design optimization can be achieved. 



\section{Nomenclature}

$$
\begin{aligned}
& \dot{\omega} \quad=\text { time derivative of speed }\left[\mathrm{rad} / \mathrm{s}^{2}\right] \\
& \omega \quad=\operatorname{speed}[\mathrm{rpm}] \\
& S \dot{S O C}=\text { time derivative of } \mathrm{SOC}[\mathrm{J} / \mathrm{s}] \\
& \text { SOC = battery state of charge [\%] } \\
& T \quad=\text { torque }[N m] \\
& S \quad=\text { number of teeth on the sun gear }[N D] \\
& R \quad=\text { number of teeth on the ring gear }[N D] \\
& K=\text { final drive ratio }[N D] \\
& m \quad=\text { vehicle mass }[k g] \\
& \text { I } \quad=\text { inertia }\left[\mathrm{kgm}^{2}\right] \\
& g \quad=\text { gravitational acceleration }\left[\mathrm{m} / \mathrm{s}^{2}\right] \\
& r_{\text {tire }} \quad=\text { radius of the tire }[m] \\
& \mu_{r} \quad=\text { rolling resistance coefficient }[N D] \\
& \rho \quad=\text { density of air }\left[\mathrm{kg} / \mathrm{m}^{3}\right] \\
& a \quad=\text { vehicle frontal area }\left[\mathrm{m}^{2}\right]
\end{aligned}
$$




$$
\begin{aligned}
& C_{d} \quad=\text { vehicle coefficient of drag }[N D] \\
& V_{o c} \quad=\text { battery open circuit voltage }[V] \\
& \eta \quad=\text { electric machine efficiency [\%] } \\
& R_{\text {batt }}=\text { internal battery resistance }[\omega] \\
& C_{\text {batt }} \quad=\text { battery capacity }[A h r] \\
& P_{\text {batt }} \quad=\text { battery power }[W] \\
& i_{\text {batt }} \quad=\text { battery current }[A] \\
& E_{p w r} \quad=\text { electric power }[\mathrm{W}] \\
& \mathrm{C} \quad=\text { torque loss }[\mathrm{Nm}] \\
& v \quad=\text { vehicle speed }[\mathrm{km} / \mathrm{h}] \\
& B S F C=\text { brake specific fuel consumption }[g / k W h] \\
& P \quad=\text { power }[W] \\
& E \quad=\operatorname{energy}[J] \\
& P \quad=\text { power }[W] \\
& i \quad=\text { current }[A] \\
& V \quad=\operatorname{volts}[V]
\end{aligned}
$$


Subscript definition:

$\begin{array}{ll}m & =\text { motor } \\ e & =\text { engine } \\ g & =\text { generator } \\ c & =\text { carrier gear } \\ r & =\text { ring gear } \\ s & =\text { sun gear } \\ s b & =\text { braking force } \\ f b & =\text { vehicle } \\ v & \\ \text { int } & =\text { actual } \\ \text { act } & \\ & \end{array}$





\section{Chapter 1}

\section{Introduction $^{1}$}

This work focuses on methods to quantify the performance of military hybrid vehicles. Chapter 1 brings together available information on military vehicle mobility drive cycles and is an expanded version of the journal article of Reference [2]. One of the noticeable omissions in the literature was attention to the electrical drive cycle which is a key element in hybrid vehicle performance evaluation. Chapter 2 introduces the notion of considering a hybrid vehicle as a microgrid and is an extended version of the conference paper of Reference [3]. This helps to shape the analysis procedure described in the subsequent chapters. Chapter 3 describes a tutorial set of drive cycles that are used in the remainder of the study that include both a mobility and electrical component. Chapter 4 describes the hybrid vehicle model used for simulation-based development of the optimal vehicle performance methods developed in Chapter 5 through Chapter 7. The method of Chapter 5 permits use of any drive cycle of interest and is an expanded version of the journal article of Reference [4]. In contrast the approach of Chapters 6 and 7 focuses on the tutorial drive cycle mentioned above. One of the benefits of this later approach is that is amenable to real-time control that could be considered in the future.

\footnotetext{
${ }^{1}$ Reprinted with permission from [2] (C) Inderscience Publishers. Letter of permission found in Appendix F
} 


\subsection{Motivation}

With ever increasing emission and fuel economy requirements in the U.S., Europe and Asia, most of the passenger car Original Equipment Manufacturers (OEMs) have conducted extensive research on various types of hybrid vehicles. The literature illustrates not only research, but includes product development; most of the OEMs in Europe and the Americas have a hybrid model in the marketplace or will introduce one in the near future [5]. Hybrid powertrain components consisting of power electronics and electric motor drives have established themselves as a means of improving the energy efficiency of passenger cars [5]. Additionally, there has been significant progress in the development of hybrid transit buses worldwide [6], which have also shown that energy savings can be realized with hybrid powertrains due to the large number of brake energy regenerative opportunities. Hybrids have also been extended to delivery trucks and garbage trucks, which have a similar application that utilizes the same type of urban drive cycle.

Militaries worldwide are also interested in realizing the potential energy savings associated with hybrid vehicles. "Fossil fuel accounts for 30 to 80 percent of the load in convoys into Afghanistan, bringing costs as well as risk. While the military buys gas for just over $\$ 1$ a gallon, getting that gallon to some forward operating bases costs \$400," according to Gen. James T. Conway, the commandant of the U.S. Marine Corps [7]. In fact, the U.S. Army has been researching hybrid vehicles since 1943 [8]. However, from observing the literature, it appears that the U.S. and other countries are far away from realizing a military hybrid ground vehicle.

There are very few, if any, military hybrid hardware related papers, and many of the papers overlook some of the basic requirements of military ground vehicles, such as $60 \%$ grade ability and fording. The lack of literature related to European and Asian military vehicles suggests that armies worldwide are also facing the challenge of fielding a hybrid military vehicle. Furthermore, a standard or universally accepted military duty cycle for measuring fuel economy does not exist. Generically a duty cycle describes a system's exchange of power with its surroundings over time; with 
respect to a vehicle a duty cycle could include mobility, usually referred to as a drive cycle or propulsion cycle, or electrical power. Lastly, the existing research fails to focus on a particular technology. This could be for the following reasons:

1. Military ground vehicle researchers do not publish as readily as OEM researchers, due to lack of available data, test vehicles and proprietary information.

2. The challenge of a military application is much greater due to the ever increasing and mutating threats that translate into continually changing vehicle requirements.

3. The life cycle of military vehicles is much different than that of passenger vehicles and not enough development has been completed to understand the longterm reliability and maintainability of hybrid components.

4. The off-highway mobility requirements, e.g. soft soil mobility, present a unique challenge and off-highway production hybrid vehicles are only recently starting to emerge in the construction equipment sector.

It is important to note that there are other potential payoffs associated with military hybrid vehicles. The first benefit is the ability to idle and possibly move without the acoustic and thermal signatures of an internal combustion engine [8]. Another benefit is the increased available on-board electrical power; not only can a hybrid system, such as an engine with an integrated starter generator, provide more electrical power than the typical alternator, but this power can be converted, conditioned and delivered in any form to and from any load. Some examples included charging the soldier's battery powered equipment or delivering power back into an electrical grid. Additionally, new military vehicles are demanding an excess of $50 \mathrm{~kW}$ of electrical power [9], which can only be provided with an advanced on-board power unit or a hybrid system. Quantifying these capabilities from an operational energy standpoint could help governments understand the benefits of military hybrid vehicles.

Electric power delivery is especially important to the U.S. Army, because their reliance on electrical power is greater than ever and the loss of battlefield electricity imposes a 
significant loss of capability and operational performance [10]. To ensure power and energy security, as well as reduce overall energy use, the concept of a microgrid has been introduced [11, 12]. A microgrid is defined as an aggregation of consumers and sources operating as a single system. It can connect to other grids or be operated as an island. Additionally, emerging vehicle-to-grid (V2G) technology has been shown to have the ability to support the microgrid as a source, but also a storage device for excess energy [13]. From a military standpoint, there is also an added benefit of temporary connectivity or network capability, which could be useful in a temporary peacekeeping or military operation.

To date, the V2G capability that comes along with a military hybrid has lacked quantifiable value, making it difficult to perform a cost / benefit analysis when trade studies are conducted. Additionally, there are many challenges related to controls and optimization for hybrid vehicles serving in a V2G capacity that need to be explored. Therefore, the objective of this dissertation is to provide a greater understanding of military hybrid vehicle from a complete operational energy perspective allowing their benefits to be realized. This dissertation will introduced the concept of the military hybrid vehicle microgrid (MHVM), facilitating the creation of a numerical optimization method for control and vehicle design. This approach is generic and expandable and, therefore, can include not only propulsion, but also electrical and stationary grid power requirements.

This dissertation is organized is the following manner. Chapter 1 will discuss the military hybrid vehicle research to date. Since drive cycles play such an important role in energy use, this chapter will include duty cycle research for passenger, commercial and military vehicles. Chapter 2 will detail the objective, concept and scope of the work. Chapter 3 will include a duty cycle discussion. The motivation and description of the notional duty cycle used for subsequent analysis is also provided. Chapter 4 will explain the vehicle model used for the analysis. Next, Chapter 5 will describe the basis function optimization with a simplified vehicle model. Chapter 6 will introduce the multiple input optimization framework and derivation for real time control and vehicle design. Finally, Chapters 7 and 8 will explain the results and conclusions, respectively. 


\section{$1.2 \quad$ Research Background}

To explore the concept of the MHVM and understand holistic energy use, it is important to review the work that has been done related to military hybrid vehicles to date. This section will therefore explore a survey of work on military hybrid vehicle energy use with special attention paid to drive cycles.

For fifty years, the U.S. military has been considering the use of electric drive technology [14]. To understand the performance of this technology, the Hybrid-Electric Vehicle Experimentation and Assessment (HEVEA) program was initiated in 2005 [14]. The goals of this program were to quantify how hybrids performed in a military environment, establish a test procedure for evaluating their performance and create a validated simulation tool for evaluating system-level performance [14, 15]. With the introduction of the Future Combat Systems (FCS) program, a series of conference papers were published by various OEMs to show hybridization capability on current vehicles using OEM specific hardware [16-26]. Additionally, the commercial sector has shown success with hybrid systems for heavy duty vehicles that have a known drive cycle, such as city buses and delivery trucks.

Currently, the three technology demonstrators for the U.S. Army's Joint Light Tactical Vehicle (JLTV) all have Integrated Starter Generators (ISGs), which are not used for propulsion, but could be expanded into mild hybrid capability with the addition of a clutch connecting the generator to the transmission and additional electric energy storage [27, 28]. Additionally, the U.S. Army's Fuel Economy Demonstrator (FED) program is creating two demonstrator vehicles: one will have an ISG only and one will be a parallel electric hybrid [29-32]. 


\subsubsection{Military Application of Hybrid Systems}

While there are significant challenges to fielding a military hybrid vehicle, there is also significant opportunity to reduce fuel consumption and provide additional capabilities to the soldier.

\subsubsection{Challenges}

There has been years of work on U.S. military hybrids. However, there has not been a military HEV fielded to date. A paper published in 2009 explains in detail the challenges that military vehicles face [8]. In summary, the vehicle performance requirements such as $60 \%$ grade ability, speed on grade, cooling and soft soil mobility add challenges that could diminish the efficiency gains seen by a hybrid vehicle. In addition, their reliability and maintainability is unknown for the life-cycle of a military vehicle. Lastly, the continuously changing threat impedes engineers from understanding the duty cycle and use of the vehicle. However, as technology advances and hybrids become mainstream for commercial applications, including some heavy duty vehicles such as buses and delivery trucks, it appears that these technologies could be leveraged to eventually field hybrid military vehicles.

\subsubsection{Opportunity}

It is generally accepted that hybrids can provide improved fuel economy. In fact, a study conducted in 1999 concluded that by just considering an engine fuel map and eliminating the inefficiencies associated with idling, vehicle braking and low engine speed part load efficiency, notable improvements could be realized as shown in Table 1.1 [1]. Note that vehicle classes are defined by gross vehicle weight (GVW), where: class III - 4,536-6,350kg, class IV - 6,351-7,257kg, class V - 7,258-8,845 kg, 
Table 1.1

Fuel savings for class III and IV trucks predicted by the study of reference $[1]$.

\begin{tabular}{|c|c|c|l|}
\hline Vehicle & $\begin{array}{c}\text { Vehicle } \\
\text { Class }\end{array}$ & $\begin{array}{c}\text { Fuel Economy } \\
\text { Improvement }\end{array}$ & \multicolumn{1}{c|}{ Method } \\
\hline Ford E-Super Duty Truck & III & $61 \%$ & $\begin{array}{l}\text { Average over Central Business } \\
\text { District (CBD), New York City } \\
\text { Bus Cycle and Commute Phase } \\
\text { Truck Cycle (COMM) }\end{array}$ \\
\hline GMC C-Series P-Chassis Truck & III & $75 \%$ & $\begin{array}{l}\text { Average Over Central Business } \\
\text { District (CBD), New York City } \\
\text { Bus Cycle and Commute Phase } \\
\text { Truck Cycle (COMM) }\end{array}$ \\
\hline Navistar 300 Series Bus & III & $35 \%$ & $\begin{array}{l}\text { Average Over Central Business } \\
\text { District (CBD), New York City } \\
\text { Bus Cycle and Commute Phase } \\
\text { Truck Cycle (COMM) }\end{array}$ \\
\hline
\end{tabular}

class VI - 8,846-11,793kg, class VII - 11,794-14,969kg, and class VIII - 14,970kg + [33]. While this work does not take into account component integration or optimal controls, it does show the potential for medium and heavy duty vehicles. Another study by Stodolsky et al. [34] showed that class III-IV trucks can obtain an average of $93 \%$ fuel economy gains over a number of urban / city cycles while class VI-VII trucks can obtain an average of $71 \%$ over the same cycles. These two papers illustrate the promise of fuel economy improvements in heavy vehicles.

\subsubsection{Vehicle and Powertrain Overview}

This section will introduce military vehicles and the hybrid powertrain configurations used in hybrid electric vehicle literature. 


\subsubsection{Vehicles}

While many different vehicles are used in worldwide operations, there are only three different military vehicles used for all of the publications: High Mobility Multipurpose Wheeled Vehicle (HMMWV), shown in Figure 1.1, Family Medium Tactical Vehicle (FMTV), shown in Figure 1.2, and Heavy Mobility Expanded Tactical Truck (HEMMTT), shown in Figure 1.3. These three vehicles span a wide range of weights from $4,536 \mathrm{~kg}$ to $14,970+\mathrm{kg}$, indicative of class III through class VII vehicles. Furthermore, design specifications and performance data related to these vehicles is readily available.

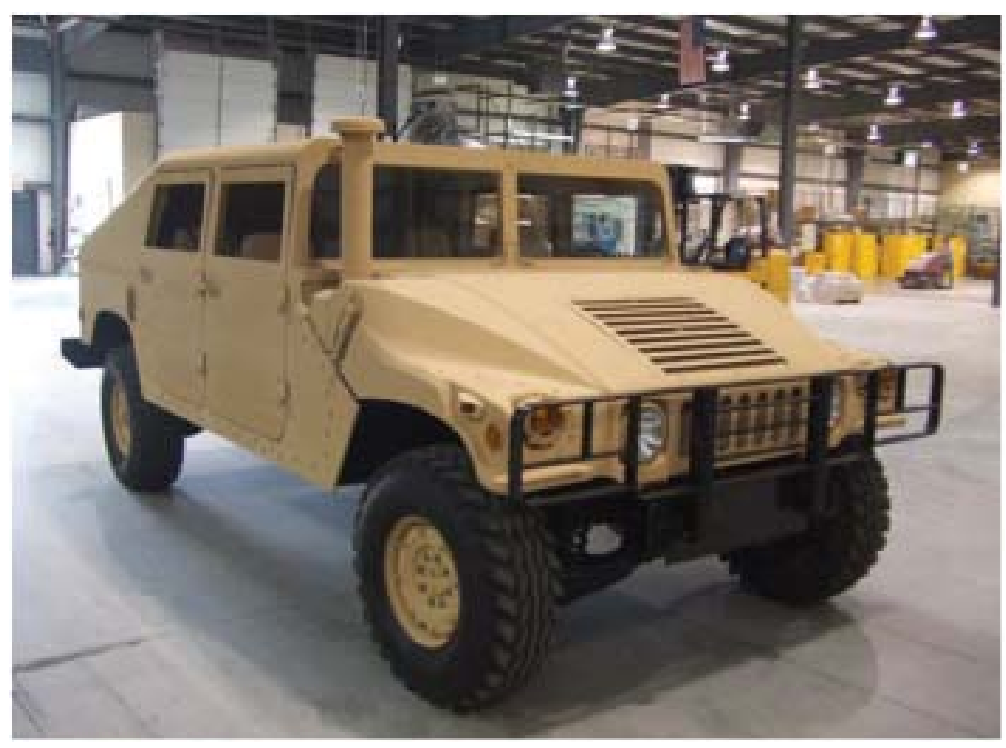

Figure 1.1: Class III HMMWV

\subsubsection{Parallel Powertrain}

A parallel hybrid powertrain is a configuration where two power sources, typically an internal combustion engine and an electric motor, propel the vehicle. This system is described by the term "parallel" because the power to move the vehicle can come 


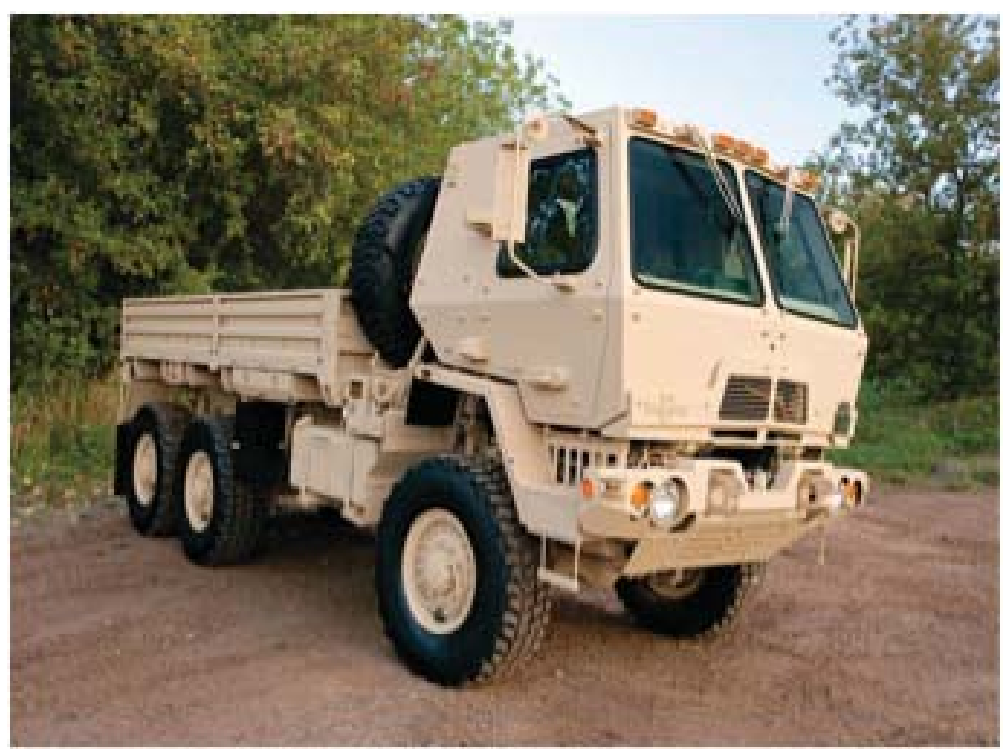

Figure 1.2: Class VI - VII FMTV

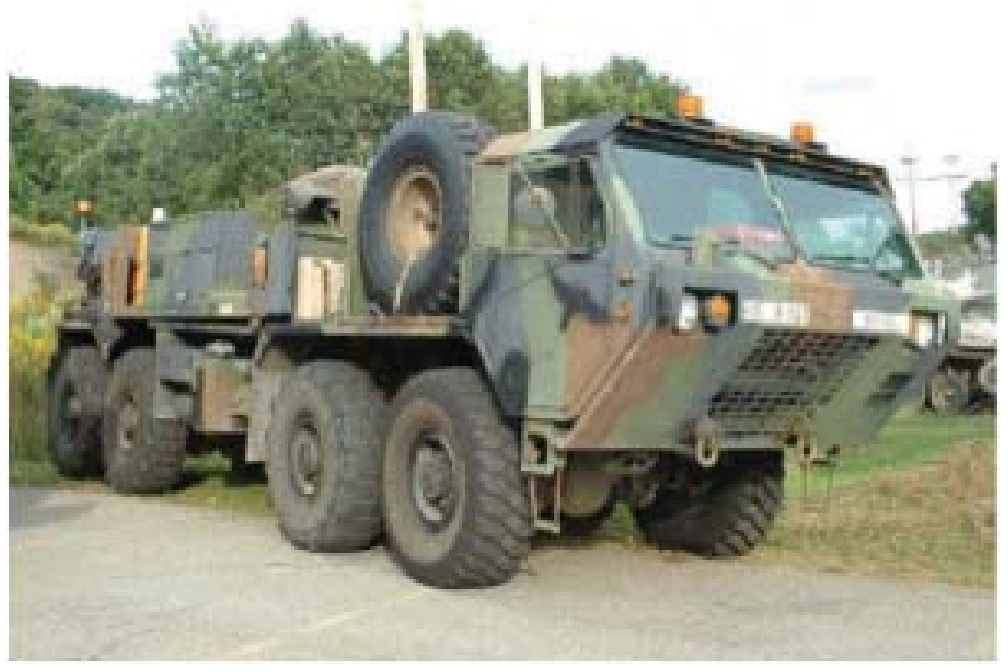

Figure 1.3: Class VIII HEMMTT

from either or both of the sources at any time. A detailed description of the different powertrain versions are explained in references [35-38]. Note that a "series-parallel" hybrid is used to describe a parallel hybrid where one source can be completely uncoupled from the second source. That first source, typically an internal combustion engine, can be used in series with the second source as a series hybrid, which is explained in the next section. 


\subsubsection{Series Powertrain}

A series powertrain is where a single device propels the vehicle, but it receives its power from additional sources. Typically, electric motors propel the vehicle using power supplied by an energy storage system, which in turn is supplied by an onboard, internal combustion engine. This system is called a "series hybrid" because propulsion power is transferred in a serial fashion from one source to the next; power is not blended from multiple sources as in a parallel hybrid. A detailed description can be found in references [35-38].

\subsubsection{Duty Cycle Overview}

In the case of simulating a mobile vehicle to determine fuel economy, it is necessary to test or simulate a vehicle over a specified drive cycle, which is also sometimes referred to as a mobility or propulsion drive cycle. A review of the literature showed that many different mobility cycles were being used to evaluate vehicle performance. These cycles can be divided into two categories: (1) time dependent speed profiles, such as the example shown in Figure 1.4, usually defined by the federal government (EPA) [39], including the FTP 75 cycle, urban cycle and the highway cycle and (2) distance dependent grade or elevation profiles, shown in Figure 1.5, usually defined by the U.S. Army, including the Churchville cycle, Harford cycle and Munson cycle.

In general, hybrid vehicle fuel savings are best realized when the vehicle undergoes frequent speed or load changes. A qualitative examination of Figures 1.4 and 1.5 shows that the FTP75, Federal Urban, Churchville and Hartford cycles all have significant speed or load frequency content. Conversely, the Federal Highway and Munson cycles have very few speed or load changes. However, an electrical duty cycle is not considered in these drive cycles. There are some nebulous references to ancillary or accessory loads in the literature, but it is not clear what types of load or cycles are being used. 


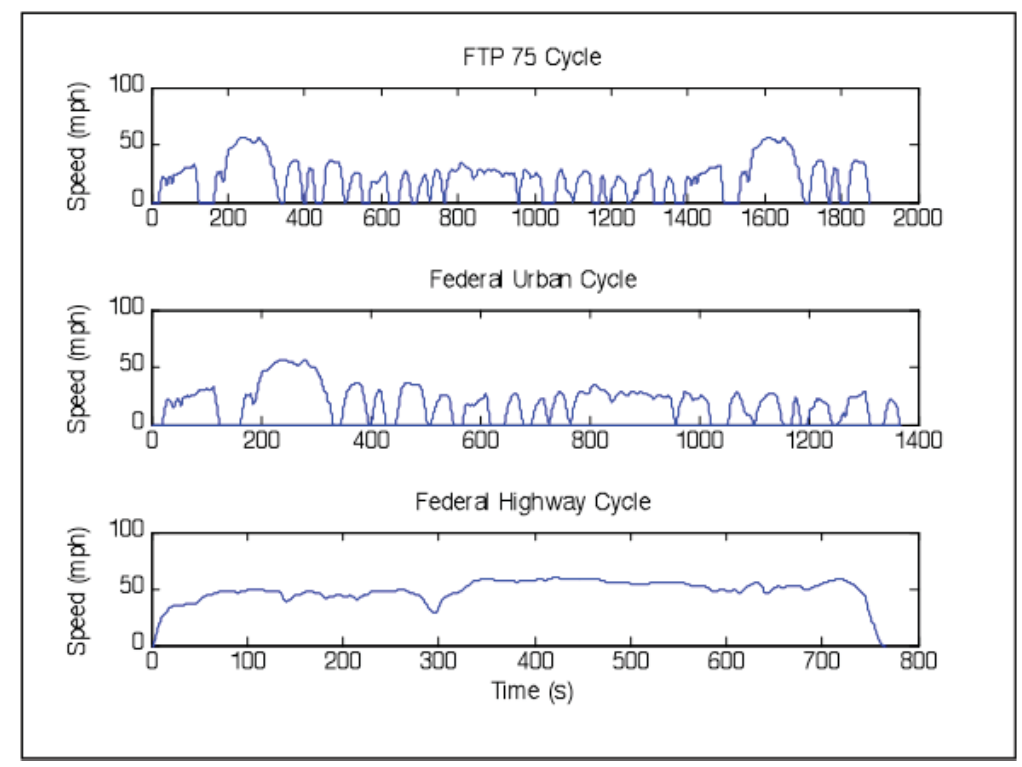

Figure 1.4: Time dependent speed profiles

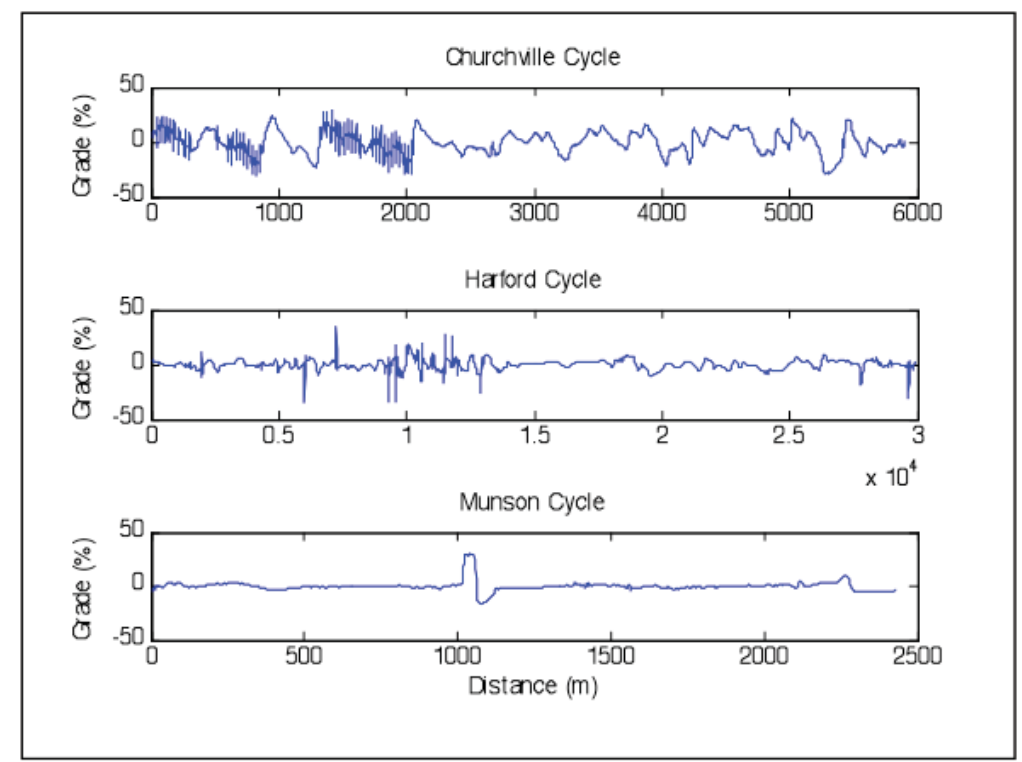

Figure 1.5: Distance dependent grade profiles

A survey of passenger and commercial vehicle drive cycle literature dating back to 1973, when Kruse [40] published the first paper detailing the definition of the federal urban cycle, omits the electrical duty cycle. A large amount of work has focused on classifying driving conditions for specific cities or countries: Australia [41], France [42], Tehran [43], New York City [44-46], Europe [47, 48], Ann Arbor [49], China [50], Seoul [51], and Palermo/Naples [52]. The other large focus is the determination of a 
generic test schedule to represent real driving conditions [53-56]. However, a survey conducted by Bata et al. [57] of real and synthesized cycles from the U.S., Canada and Japan, conluded that synthesized cycles are better for testing purposes, but do not represent real world driving. Approaching the problem from a new direction, Rykowski et al. [58], introduced a model and tool to quantify fuel consumption that was drive cycle invariant. O'Keefe et al. [59], introduced the "hybrid advantage" calculation, which characterizes a duty cycle's suitability for hybrid vehicle usage. Along these same lines, Zou et al. [60], determined which cycle was relatively insensitive to battery state of charge. However, neither publication considered electric or ancillary loads in their analysis.

From a military perspective, Brudnak et al. [61] and Dembski et al. [62] attempt to characterize a military drive cycle, but once again the electrical cycle is omitted. Based on the surveyed literature, these military cycles have not been adopted by the community.

\subsubsection{Documented Fuel Economy Improvements}

One of the major advantages of a hybrid vehicle is its ability to recoup energy normally lost in a braking event. This is typically referred to as regenerative braking. If the duty cycle only consists of steady state operation, then the braking events would be minimized, which would not allow the full benefit of the hybrid vehicle to be realized. This section will quantify this effect and summarize the duty cycle influence on fuel economy.

\subsubsection{Parallel Powertrain}

For parallel hybrid configurations, a class III HMMWV can realize between 4.3$45.2 \%$ fuel economy improvement depending on power system design and drive cycles, 
whereas the class VI and VII FMTV can realize between 2-32\% and 7-15\% respectively. Lastly, the class VIII HEMMTT only demonstrates an improvement between $0-2 \%$. The results of these studies [2] indicate that for parallel hybrid powertrains there exists more opportunity for fuel efficiency improvement in smaller class vehicles. A detailed list of fuel economy improvements along with the methodology used for assessment and power system design can be found in [2].

\subsubsection{Series Powertrain}

For a series hybrid configuration, a HMMWV can realize between 7-68\% fuel economy improvement depending on its power system design and drive cycles, whereas the FMTV only realizes between $-5.9-30 \%$ and $-1.5-19.2 \%$ for class VI and VII, respectively. The HEMMTT can demonstrate between $12.5-17.4 \%$ and $0-15.8 \%$ improvement for class VII and VIII, respectively. Last, a notional military bus (class VI) showed a 12.5\%-19.1\% improvement, again depending on drive cycle and technology. The series hybrid analysis, as with the parallel hybrid cases, demonstrates the greatest opportunity for efficiency improvement with lighter vehicles. However, the series hybrid shows more potential for improvement in the very large class VII-VIII vehicles than a parallel hybrid. A detailed list of fuel economy improvements along with methodology and technology can be found in [2].

\subsubsection{Drive Cycle Impact}

To further understand the effect of drive cycles, Figure 1.6 shows cycle versus percent fuel economy improvement for series, parallel and series-parallel combinations for the class III HMMWV vehicle based on the results provided in references [63-68], and [69]. While the configuration and methods were different for each of the points on the plot, a general trend shows that the hybrid HMMWVs show more improvement on urban cycles, which is expected as mentioned above in Section 1.2.1.2. Furthermore, vehicles tested on the Munson cycle show the least amount of fuel economy improvement, 
which is also anticipated since the Munson drive cycle is nearly a flat course without any stops as shown in Figure 1.5.

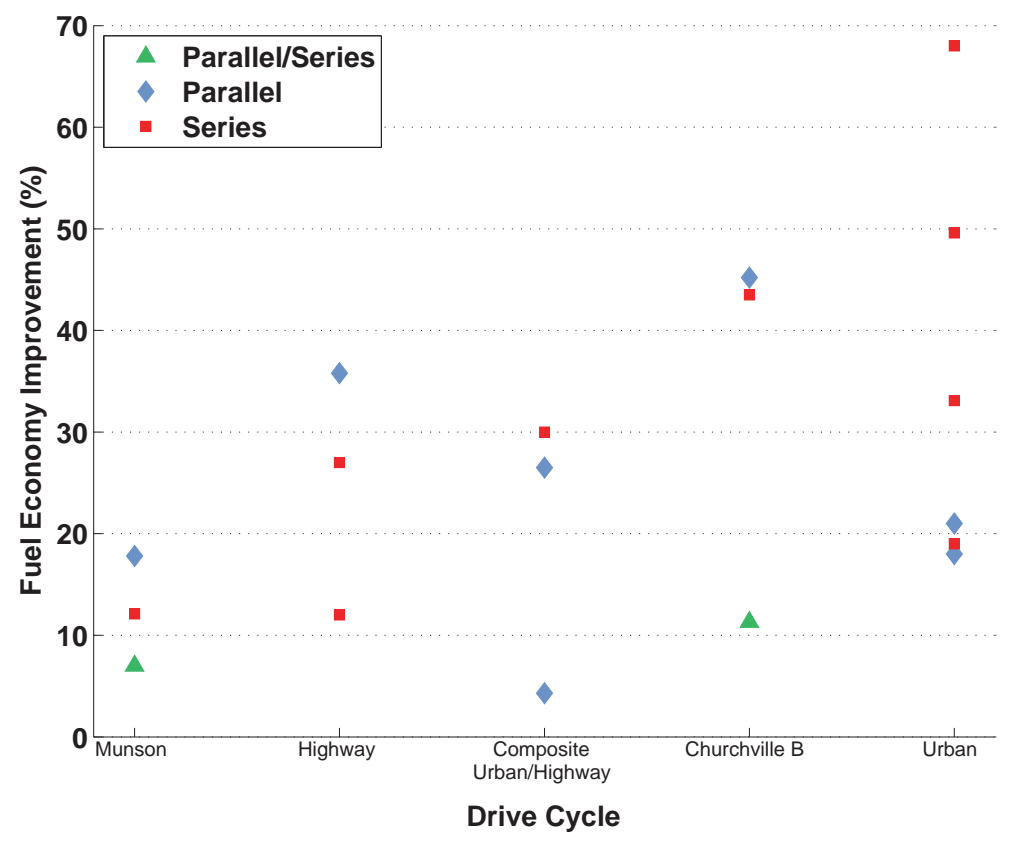

Figure 1.6: Cycle vs. fuel economy improvement for the HMMWV

Figure 1.7 is a similar plot for Class VI vehicles where the data is extracted from references [65, 70-73], and [74]. In these plots, the "Composite Urban/Highway" bin captures other ad-hoc cycles. Once more, the urban cycle shows the most improvement, while the Munson cycle shows a degradation in fuel economy in some cases.

In summary, the fuel economy improvement for military hybrid vehicles is highly dependent on the drive cycle used for the analysis. The existing literature shows a lack of a standard drive cycle for analysis, which makes it difficult to judge technologies and understand how the military can benefit from a hybrid vehicle. In addition, the concept of an electrical duty cycle is completely omitted. This is likely one of the reasons for the delay in fielding a military hybrid. 


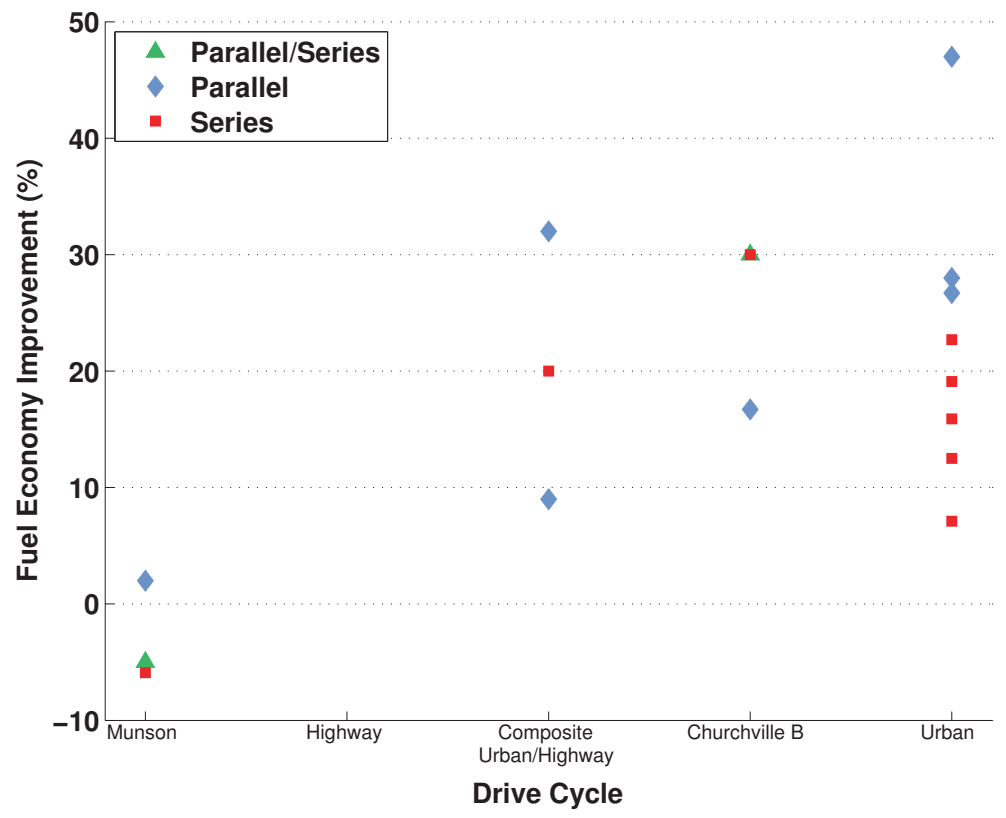

Figure 1.7: Cycle vs. fuel economy improvement for the class VI vehicle

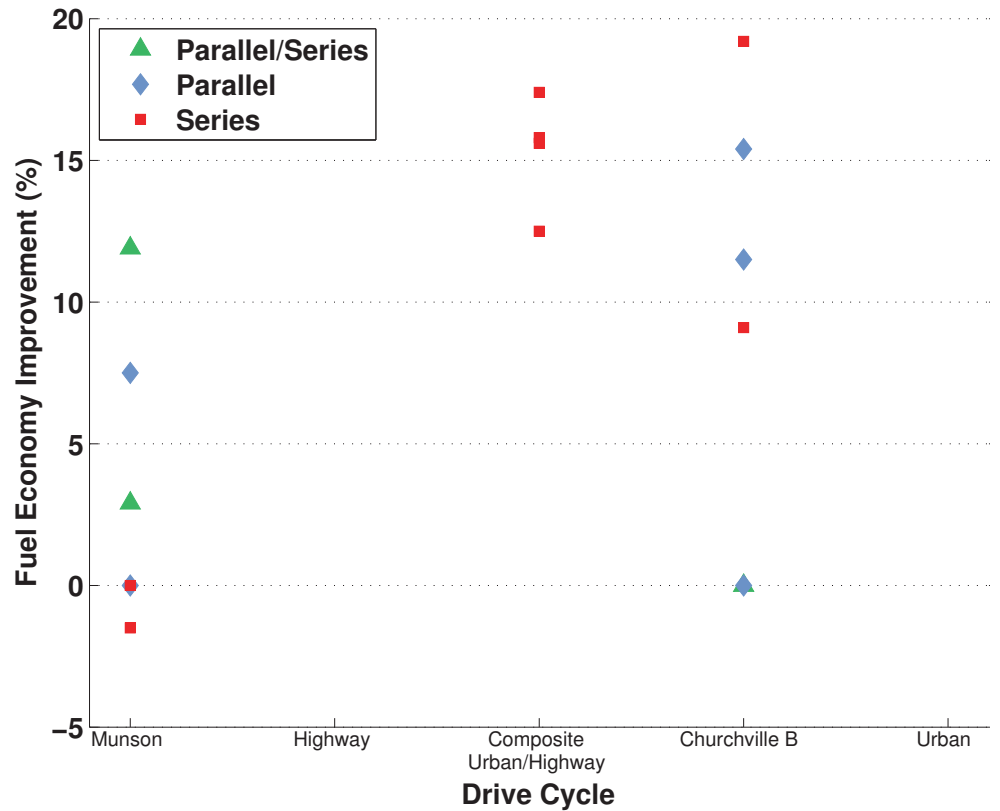

Figure 1.8: Cycle vs. fuel economy improvement for the class VII \& VIII vehicle 


\subsection{Summary}

Many studies have shown that hybrid powertrains can yield fuel economy improvement in varying types of vehicles. A survey of military hybrid peer reviewed publications illustrates that extensive work has been done with regard to their simulation, optimization and controls. All of the literature focuses on three military vehicles: HMMWV, FMTV and HEMMTT, which span equivalent commercial vehicle class III through class VIII. However, there are very few publications with respect to military hybrid vehicle hardware $[65,68,75]$, which could be due to cost, proprietary information or the fact that military hybrid vehicle hardware requires more development time than passenger vehicles. Additionally, military vehicles provide unique challenges such as a $60 \%$ grade ability, speed on grade, cooling and soft soil mobility.

Many different types of duty cycles were used for the fuel economy investigations. They include time and speed dependent cycles that are defined by the U.S. EPA and distant dependent grade profiles that are defined by the U.S. Army. Both types have duty cycles that represent urban style driving (FUDS, Churchville B) and highway style driving (Federal Highway Cycles, Munson). In addition, some of the publications used a mix of these cycles so that the fuel economy improvements are reported over a composite duty cycle. While the U.S. Army has tried to define an appropriate military drive cycle, overall there is a lack of an accepted duty cycle to estimate fuel economy improvements such as the FTP 75 used to report miles per gallon for passenger vehicles in the U.S. This could be due to the fact that military threats are constantly changing and it is generally unknown where a military vehicle will be needed.

Fuel economy analyses show that the class III vehicle had the greatest potential for fuel economy improvements over an urban cycle and that those improvements diminish with composite and highway cycles. Heavier vehicles demonstrate the same trend with respect to drive cycles. In some cases there was even a fuel economy degradation over flatter cycles, such as the Munson cycle. In general, heavier vehicles do not show as much fuel economy potential as the class III vehicles. Lastly, fuel 
economy gains are not the only capability that hybrid system can provide a military vehicle. The hybrid system can be used to provide electrical power for soldiers and allow for an improved acoustic and thermal signature.

In summary, the lack of hardware related research depicts the challenges that a military hybrid vehicle faces. Additionally, the absence of a standard method for quantifying the benefit of a military hybrid vehicle makes the cost / benefit relationship impossible to understand. Finally, the lack of quantifiable value of "other" capabilities, such as silent watch or V2G connectivity, overlooks the complete advantage that a military hybrid vehicle could provide. 



\section{Chapter 2}

\section{Concept}

The objective of this dissertation is to provide a method to assess military hybrid vehicles from a complete operational energy perspective, allowing the benefit of a military hybrid vehicle to be realized. This objective has facets. Therefore, this chapter will explain the research objective in detail. In addition, it will define the scope of the dissertation and what will be shown. Lastly, it will define MHVM, which was essential to the methods developed in this dissertation.

\subsection{Research Objective and Scope}

The creation of MHVM analysis techniques includes taking into account propulsion power, electrical power for government furnished equipment (GFE), V2G energy exchange, and V2G energy storage requirements. Grasping the dependency of the vehicle performance on these interconnected requirements allows for a comprehensive, realistic analysis and therefore the benefit of a military hybrid vehicle can be fully quantified. Additionally, it would introduce and 
explore the novel use of a vehicle as a microgrid, which is a generic and expandable concept that could be used for any propulsion system architecture. This would not only include developing methods for energy optimization, but also creating duty cycles that would represent power demand profiles related to mobile energy exchange and storage.

An unexplored challenge related to this type of analysis is how to coordinate the energy use of the vehicle with stationary microgrids to achieve an overall efficiency. In addition, a military vehicle is used in ways that provide unique challenges, e.g. electrical energy requirements for GFE, which can be weapons, communication systems, or other military specific equipment with loads can be in excess of $50 \mathrm{~kW}[9]$. This requires the consideration of a electrical duty cycle when developing vehicle controls and designing components. Furthermore, this suggests it is beneficial to treat a military hybrid vehicle as a microgrid and utilize energy optimization methods from stationary microgrids. Therefore, the scope of this dissertation is to create an optimization framework that allows for optimal control and design of military hybrid vehicles while considering multiple vehicle power requirements.

\subsection{Microgrid Introduction}

Figure 2.1 illustrates typical components of a stationary microgrid as originally defined by Lasseter in reference [11]. It is defined by an energy generator, consumer and storage device. As shown in Figure 2.1, a generator can be any technology that can feed energy to the grid, a consumer is the user of this energy and the storage device stores excess energy when available or provides energy when necessary to offset generator/consumer mismatch or as part of an optimal management scheme. A supervisory control may be used to oversee the energy transfer, thus ensuring that all requirements are met in the most efficient manner possible. Localized control schemes, such as droop control, can also be used to facilitate power flow utilization of distributed generation assets. 


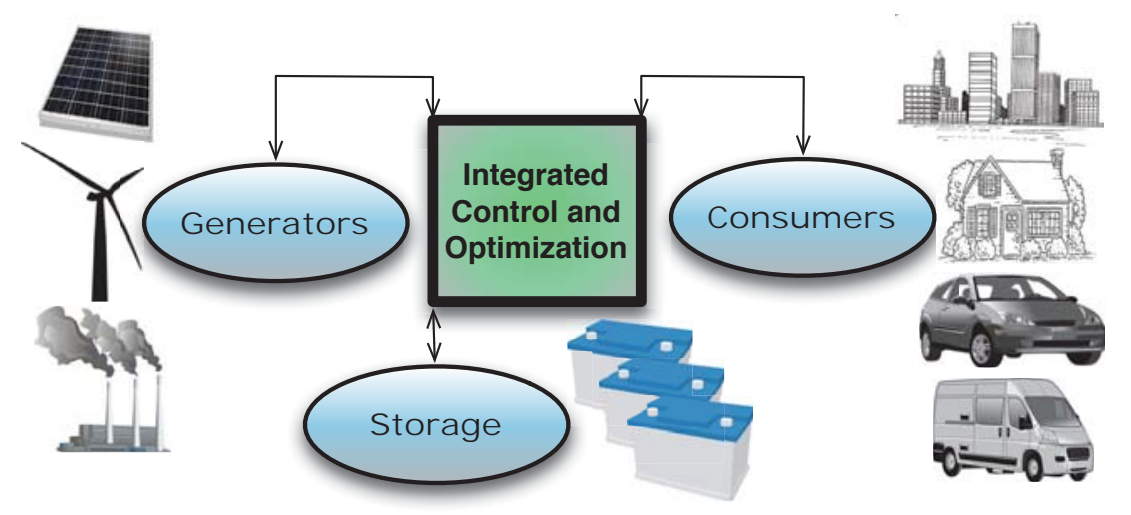

Figure 2.1: Overview of a generic stationary microgrid

From a military microgrid perspective, it has been shown that state of charge $(S O C)$ control and design optimization can reduce fuel use from 3 to $30 \%$, due to downsized generators and control of renewable energy with a forward looking energy storage strategy [76]. Peters, et al. [77] used model predictive control to solve the power dispatch problem for a military microgrid using various prediction horizons. This work also determined that limitations in batteries led to energy waste and the design of microgrids would benefit from more effective control and design of the battery system. The effect of the battery resistance was investigated with respect to voltage and frequency regulation and it was determined that an effective inverter based control design should depend on both regulation and the direct current (DC) source characteristics [78]. Lastly, it was illustrated that a range of plug-in hybrid electric vehicle penetration levels can satisfactorily regulate the voltage and frequency of a military microgrid [79]. In all of this work, storage control and design optimization played a large role.

The concept of a microgrid can be applied on any scale, e.g. a large city or a single building, therefore it should also be applicable to a military ground vehicle as shown in Figure 2.2. It has a source (typically an internal combustion engine) and consumer (i.e. the propulsion requirement or GFE) and storage (usually a battery of some type). Additionally, its goal is similar to a microgrid - to fulfill power requirements in the most efficient manner possible. Therefore, the supervisory control of the vehicle would benefit from exploiting methods used to optimize stationary microgrid performance, 
namely the SOC optimization, which has yet to be explored from a vehicle standpoint.

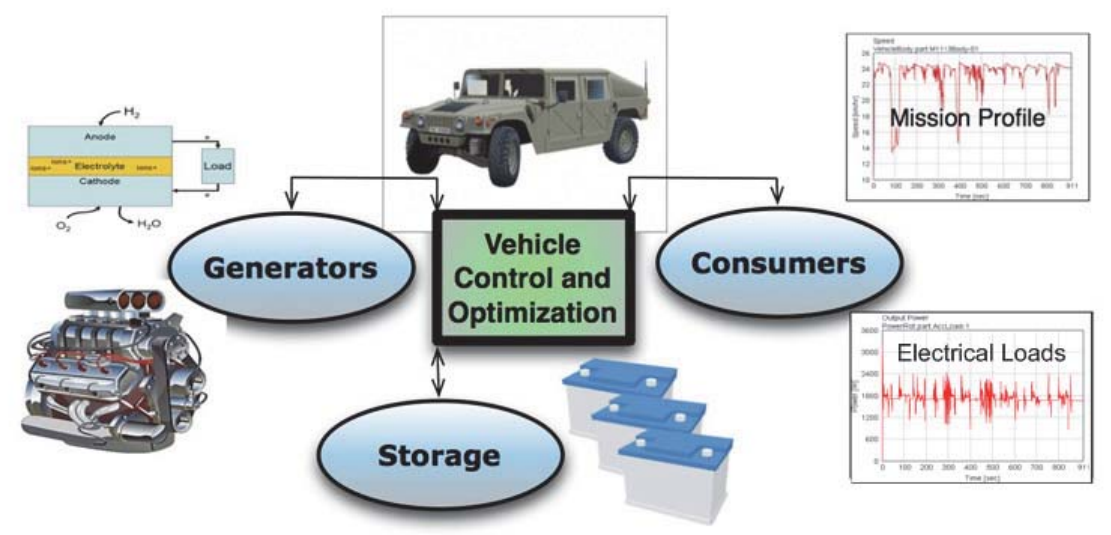

Figure 2.2: Overview of a vehicle microgrid

This concept becomes increasingly advantageous when the vehicle has the ability to plug into another microgrid and either absorb or provide power; this is described by the term "vehicle-to-grid (V2G) connectivity." As shown in Figure 2.3, the vehicle now has multiple sources, the engine and the microgrid, and multiple consumers, the propulsion requirements and the microgrid. This capability also allows the military an added security element to temporarily connect microgrids via a hybrid vehicle or utilize the vehicles as the sole source for a microgrid in the event a different source was removed or unable to provide enough power.

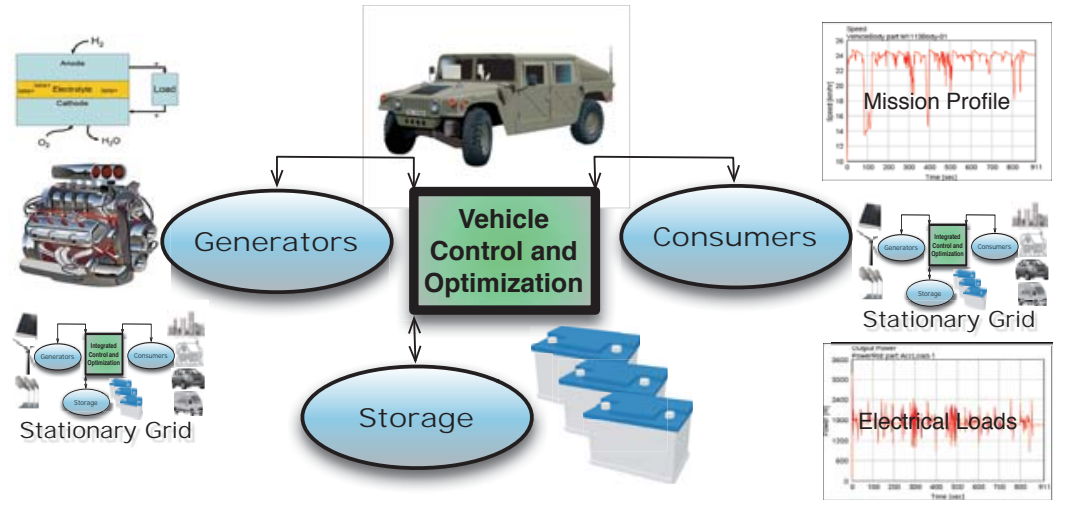

Figure 2.3: Overview of a vehicle integrated into a stationary microgrid 


\section{Chapter 3}

\section{Duty Cycles and Their Adaptation}

\section{to Military Hybrid Vehicles}

As explained in Chapter 1, a standard military hybrid propulsion duty cycle has not been developed or agreed upon and the concept of an electrical duty cycle has been neglected in the previous literature. Furthermore, the consideration of the stationary microgrid requirement has yet to be explored. This chapter will explain the duty cycles used for the analysis and the reasoning behind them.

\subsection{Propulsion Cycle}

Recall that the performance of a military hybrid vehicle shows the most benefit on an urban drive cycle as shown in Figure 3.1 [2], which is a plot of drive cycle versus fuel economy improvement. 


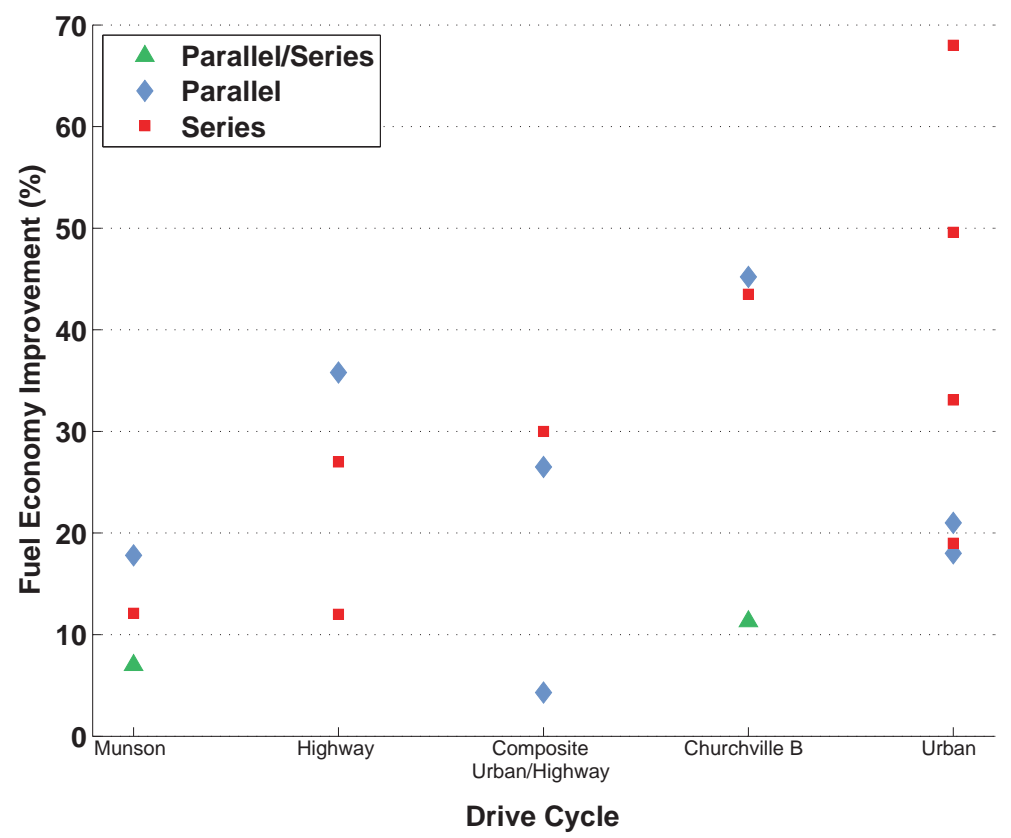

Figure 3.1: Cycle vs. fuel economy improvement for the HMMWV (originally shown in Chapter 1)

To recognize the full benefit of a military hybrid vehicle, an urban style propulsion duty cycle, which consists of varied speed with periods of steady state operation is needed. Thus, a duty cycle of vehicle speed, $v(\mathrm{~km} / \mathrm{h})$, versus Time (s) was developed and shown in Figure 3.2. This cycle contains the relevant urban drive cycle features and the results can be extrapolated to longer cycles. In addition, the brevity of it allows the complex details and results to be apparent. A more complex, longer duty cycle can be readily created from this kernel by linear combination of time shift, amplitude scaling and time scaling.

\section{$3.2 \quad$ Electrical Cycle}

Military vehicles are equipped with government furnished equipment (GFE), loosely defined as communication devices, weapons systems or any other military specific item. Many of these systems require large $(500 \mathrm{~W}-5000 \mathrm{~W})$ electrical power amounts 


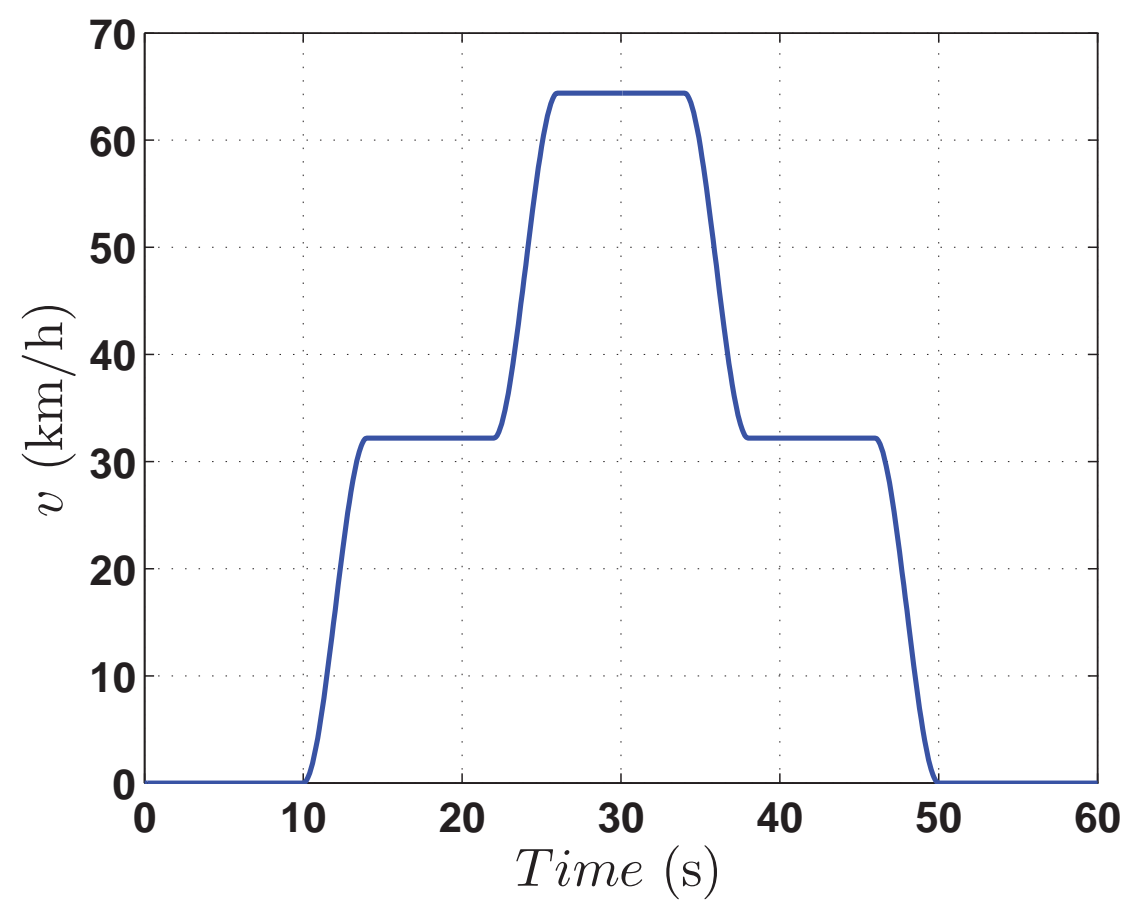

Figure 3.2: Propulsion duty cycle

for short periods of time $(1 \mathrm{sec}-1 \mathrm{~min})$. The current vehicle design process sets the electrical load requirement using the assumption that the GFE is always on. This overly conservative approach leads to increased vehicle weight and fuel consumption. A rational analysis strategy does not exist for exploring electrical duty cycle impact on vehicle design. The optimization approach described in Chapter 6 addresses this gap.

The three electrical duty cycle cases are shown in Figure 3.3 over the 60 second mobility event of Figure 3.2. The constant 300W load represents a nominal set of GFE that would typically be active during a mobility drive cycle. The $600 \mathrm{~W}$ pulse load represents the activation of a GFE load that can occur at any point during the mobility drive cycle. The $900 \mathrm{~W}$ constant load represents the current approach to vehicle design where the $600 \mathrm{~W}$ load is assumed to be active during the entire mobility drive cycle.

These electrical duty cycles will be overlaid on the mobility cycle, so that operational 


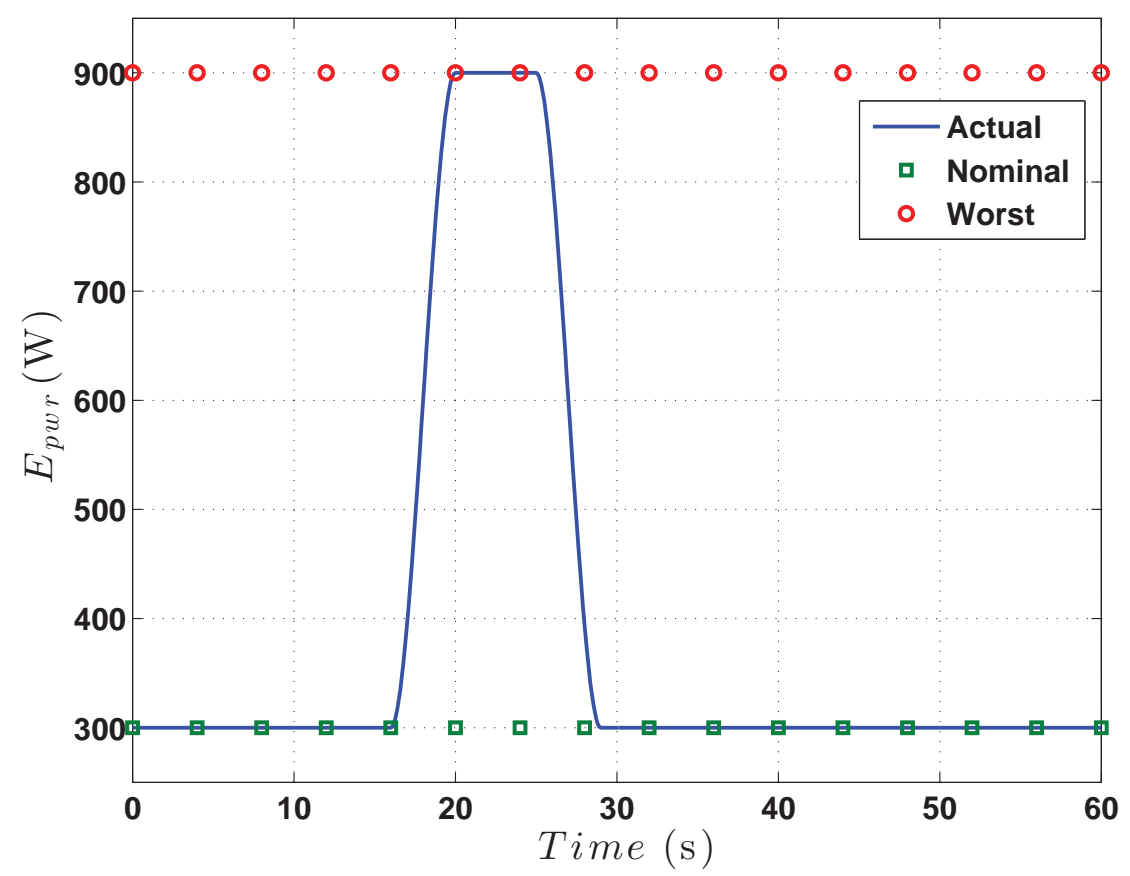

Figure 3.3: Electrical duty cycles

energy requirements are realized. Chapter 7 will display the effects of these different electrical cycles and how important they are in controls development and system design.

\subsection{Stationary Microgrid}

Finally, to complete the energy analysis, stationary vehicle-to-grid (V2G) requirements will be considered. The following scenario is of particular interest to the U.S. Army. Consider the case where a vehicle approaches a stationary microgrid, as when a vehicle returns to base after a mission. The stationary microgrid control system will be optimizing its stationary asset utilization, including its $S O C$, when a vehicle microgrid communicates with the stationary microgrid that it is returning to base. The vehicle will soon become another device connected to the stationary microgrid 
and could behave as a load, generation, or energy storage. The stationary microgrid's control system, likely including a continuous optimization scheme, would then determine how the vehicle can be best integrated. The stationary microgrid is assumed to communicate to the vehicle a desired connect state, including its $S O C$. Therefore, the vehicle knows it's desired $S O C$ at the end of the mission, which is the same as its docking time with the stationary microgrid and is denoted as $S O C_{\text {final }}$. This parameter will be considered as part of the duty cycle. Consequently, vehicle speed, $v$ $(\mathrm{km} / \mathrm{h})$, electrical power $E_{p w r}(\mathrm{~W})$, and $S O C_{\text {final }}(\%)$, uniquely defines the combine drive and electrical duty cycle used for the subsequent analysis. 



\section{Chapter 4}

\section{Vehicle Model}

As explained in Chapter 1, a military hybrid has yet to be fielded, but several demonstrators have been built and modeled over the years. Most, if not all, of the vehicle data is either classified or intellectual property of a defense contractor. In addition, since these vehicles were one-off systems, they are far from design optimal. Therefore, it proves to be very challenging to create or obtain a reasonable military hybrid vehicle model that can be used for optimization and controls. Furthermore, the scope of this work is to create an optimization framework that will allow for optimal control and design of a military hybrid vehicle while considering all vehicle power requirements. This framework should be applicable to any hybrid system, which is also one of the benefits MHVM. Therefore, it was determined that the vehicle model used for this dissertation should be a commonly used hybrid architecture with an internal combustion engine and one or more electric machines, as close to design optimal as possible, without any publication restrictions.

The Toyota ${ }^{\circledR}$ Hybrid Prius, which contains an internal combustion engine and two electric machines, has been in the marketplace since 1997, when the first generation was launched [80]. The Prius has been in service the longest amount of time if any hybrid vehicle. The third generation, which became available in 2010, has an 
abundance of data and research available in the public domain. All of this makes it an ideal candidate for this work. This chapter will detail the Prius vehicle model and the parameters utilized for this research.

\subsection{Overview}

The vehicle model used for development of the optimization-based analysis approach is the Toyota ${ }^{\circledR}$ Prius hybrid system, shown in Figure 4.1, which combines a series hybrid with a parallel hybrid to realize the advantage of both systems. It contains an internal combustion engine, a generator and an electric motor. The electric motor can be used for regenerative braking and to propel the vehicle, while the generator is used to charge the battery and to transfer power from the internal combustion engine to the electric motor. Lastly, the internal combustion engine can directly drive the wheels via the motor when necessary.

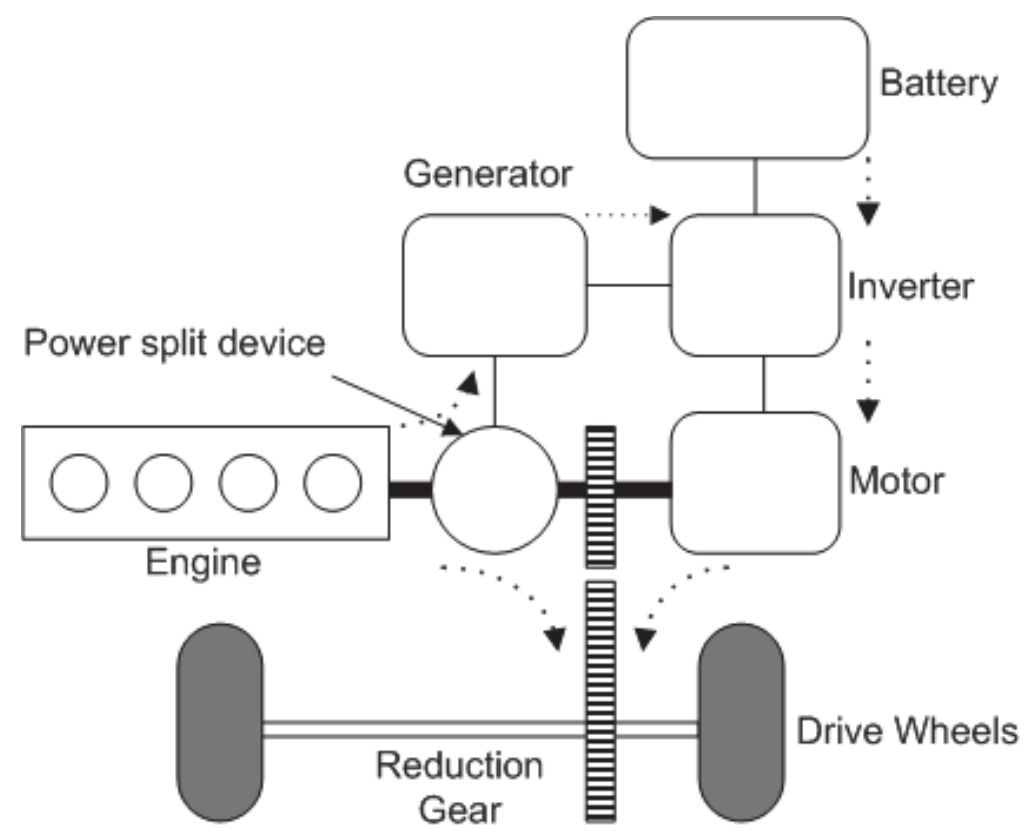

Figure 4.1: Power split overview 
The Prius powertrain configuration consists of a planetary gear set that has three components, the ring gear, the sun gear and the carrier gear, which are connected to the motor, generator and engine, respectively. This allows for engine propulsion power to be utilized via a mechanical path or an electrical path. The mechanical path includes transferring power through the gear system to the motor. The electrical path transfers power from the engine through the generator to the motor. The kinematic relation through the gear system is described by [82]:

$$
\omega_{g} S+\omega_{m} R=\omega_{e}(R+S)
$$

Due to this relationship, there are only two degrees of freedom in the speed domain, but the engine torque, generator torque and engine torque can all be specified independently. Therefore, two states of the system are defined as motor speed, $\omega_{m}$, and engine speed, $\omega_{e}$. The two dynamic equations for these states are shown in Equations (4.2), (4.3) taken from [81] and [82].

$$
\begin{aligned}
& {\left[\frac{I_{v}^{\prime}(R+S)^{2}}{I_{e}^{\prime} K R}+\frac{I_{v}^{\prime} S^{2}}{I_{g}^{\prime} K R}+R\right] \dot{\omega}_{m}(t)=} \\
& T_{m}(t)\left[\frac{(R+S)^{2}}{I_{e}^{\prime} R}+\frac{S^{2}}{I_{g}^{\prime} R}\right]+T_{e}(t)\left[\frac{(R+S)}{I_{e}^{\prime}}\right]+T_{g}(t)\left[\frac{S}{I_{g}^{\prime}}\right]-C\left[\frac{S^{2}}{I_{g}^{\prime} K R}+\frac{(R+S)^{2}}{I_{e}^{\prime} K R}\right] \\
& {\left[(R+S)+\frac{I_{e}^{\prime} S^{2}}{I_{g}^{\prime}(R+S)}+\frac{I_{e}^{\prime} K R^{2}}{I_{v}^{\prime}(R+S)}\right] \dot{\omega}_{e}(t)=} \\
& T_{m}(t)\left[\frac{K R}{I_{v}^{\prime}}\right]+T_{e}(t)\left[\frac{S^{2}}{I_{g}^{\prime}(R+S)}+\frac{K R^{2}}{I_{v}^{\prime}(R+S)}\right]-T_{g}(t)\left[\frac{S}{I_{g}^{\prime}}\right]-C\left[\frac{R}{I_{v}^{\prime}}\right]
\end{aligned}
$$


Where the component inertias are defined as:

$$
\begin{aligned}
I_{v}^{\prime} & =I_{m} K+I_{r} K+\frac{m r_{\text {tire }}^{2}}{K} \\
I_{g}^{\prime} & =I_{s}+I_{g} \\
I_{e}^{\prime} & =I_{c}+I_{e}
\end{aligned}
$$

The quantity, $C$, captures the friction and aerodynamic parasitic losses and the brake forces.

$$
C=T_{f b}+r_{t i r e} m \mu_{r} g+\frac{0.5 C_{d} r_{t i r e}{ }^{3} a \rho \omega_{m}(t)^{2}}{K^{2}}
$$

The last state, $S O C$, can be determined by:

$$
S \dot{O} C=-\frac{i_{b a t t}}{C_{b a t t}}
$$

Where the power in the battery can be represented by an internal resistance model through Equation (4.10).

$$
P_{b a t t}=V_{o c} I_{b a t t}-i_{b a t t}^{2} R_{b a t t}
$$

However, for this vehicle configuration, the power in the battery can be described in terms of torque, $T$, and speed, $\omega$, of the motor and generator with Equation (4.11).

$$
P_{b a t t}=T_{m} \omega_{m} \eta_{m}-T_{g} \omega_{g} \eta_{g}
$$

Combining Equations (4.9), (4.10), and (4.11), the third state, SOC, can be calculated 
through Equation (4.12).

$$
S \dot{O} C(t)=\frac{-V_{o c}+\sqrt{V_{o c}^{2}-4 R_{\text {batt }}\left(T_{m} \omega_{m} \eta_{m}-T_{g} \omega_{g} \eta_{g}+E_{p w r}\right)}}{2 R_{\text {batt }} C_{\text {batt }}}
$$

Now the vehicle can be modeled using a three-state system of Equations (4.2), (4.3), and (4.12). The states are the vehicle's motor speed, $\omega_{m}$, the vehicle engine speed, $\omega_{e}$, and the battery system $S O C$. The inputs to the model are the motor, engine and generator torques denoted as $T_{m}, T_{e}$, and $T_{g}$.

\subsection{Internal Combustion Engine}

The internal combustion engine found in the Toyota ${ }^{\circledR}$ system is described as a $1.8 \mathrm{~L}$ four cylinder engine with a maximum torque of $115 \mathrm{Nm}$ at $2200 \mathrm{rpm}$ [80]. Since data was available for a similar sized engine, it was decided that this data would be scaled down to match the Toyota ${ }^{\circledR}$ engine. Once the data from the larger engine was scaled down, the torque curve, shown in Figure 4.2, and the fuel map, shown in Figure 4.3 were created. Figure 4.2 shows the maximum engine torque $(\mathrm{Nm})$ available at each engine speed $(\mathrm{rpm})$. Figure 4.3 shows the brake specific fuel consumption $(\mathrm{g} / \mathrm{kWh})$ at each engine load $(\mathrm{Nm})$ and speed $(\mathrm{rpm})$, which is used to calculate the fuel used. Both of these plots show good agreement to published plots of the torque curve and fuel map for the Toyota ${ }^{\circledR}$ system [83]. The torque curve of Figure 4.2 has the same shape and the peak location as the published curve [83]. The fuel map plot [83] was vague, but it showed a trend of increased fuel consumption at lower engine speed, which agrees with the scaled fuel map. 


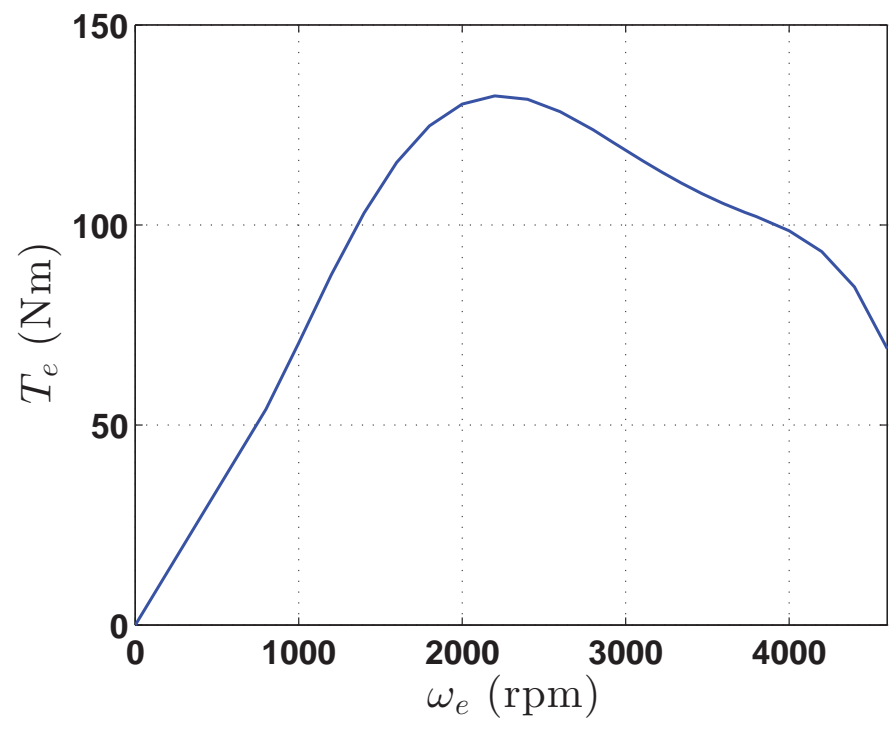

Figure 4.2: Engine torque curve

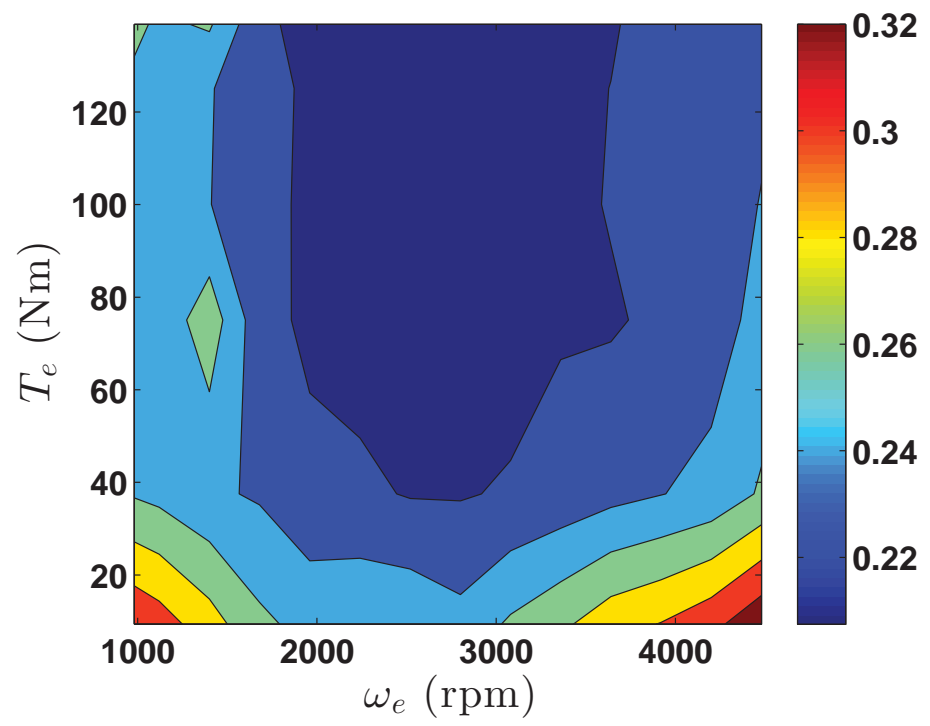

Figure 4.3: Engine fuel surface $(g / k W h)$ 


\subsection{Electric Machine Performance}

The motor and generator found in the Toyota ${ }^{\circledR}$ system have a maximum torque of $400 \mathrm{Nm}$ and $75 \mathrm{Nm}$, respectively. The torque curve is defined as motor or generator torque $(\mathrm{Nm})$ at each motor or generator speed $(\mathrm{rpm})$. The efficiency map is the efficiency at each each motor or generator load $(\mathrm{Nm})$ and motor or generator speed $(\mathrm{rpm})$, which is used to calculate actual power. Instead of using the Toyota ${ }^{\circledR}$ electric machine performance characteristics, available data for a larger system was scaled to match the Toyota's ${ }^{\circledR}$ maximum torque and efficiency characteristics. The motor torque curve is shown in Figure 4.4 and generator torque curve is shown in Figure 4.5.

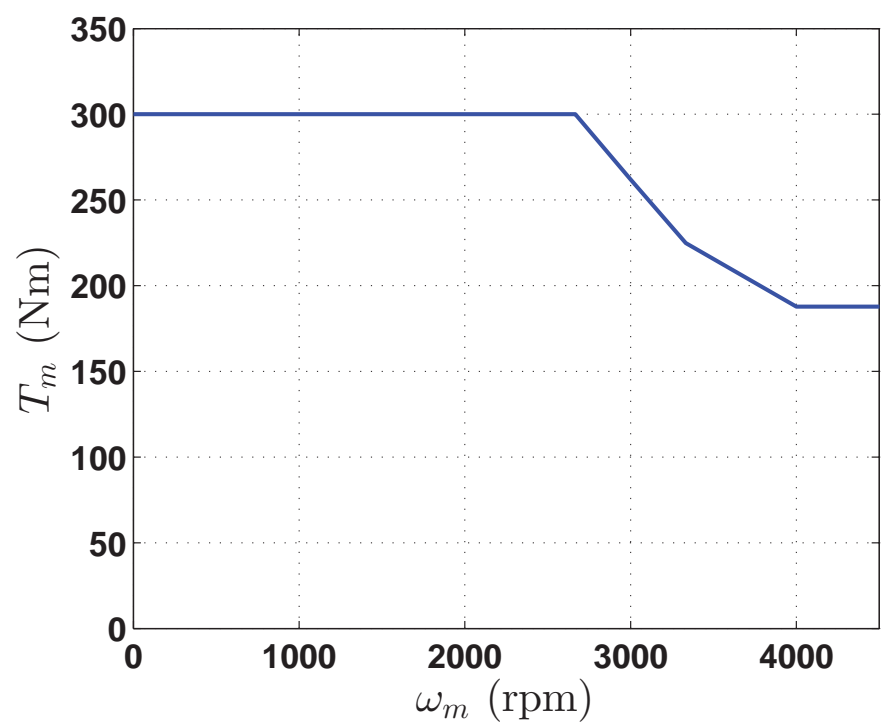

Figure 4.4: Motor torque curve

The motor efficiency map is shown in Figure 4.6 and generator efficiency map is shown in Figure 4.7. These curves were compared to the motor and generator performance curves for the Toyota ${ }^{\circledR}$ system found in an evaluation report performed by the U.S. Department of Energy [80].

More specifically, the scaled torque curves had the same shape and the torque fell 


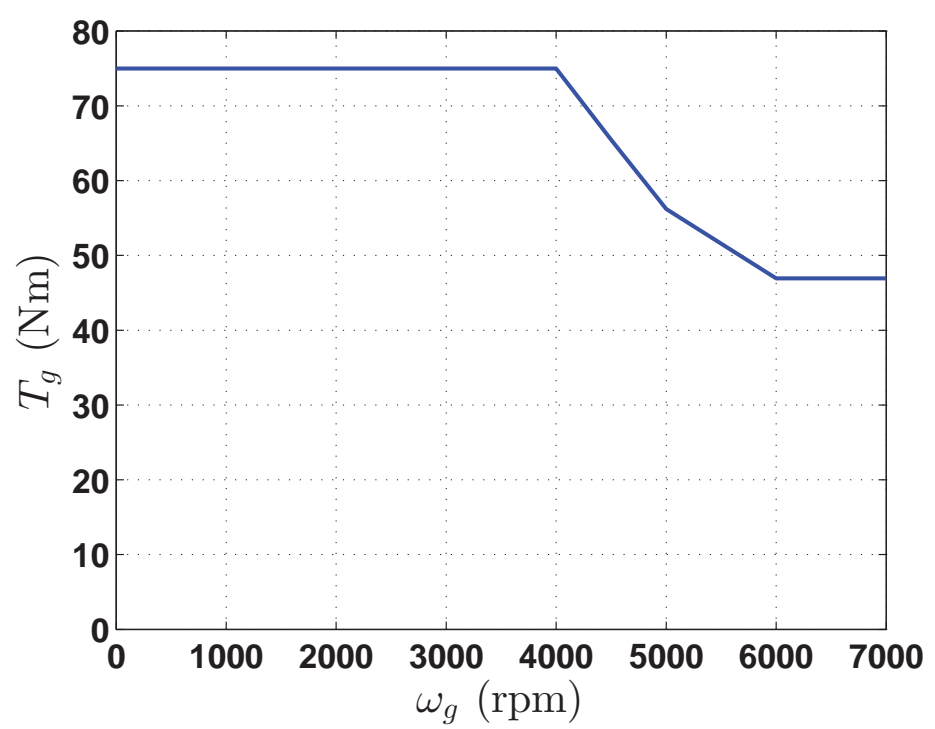

Figure 4.5: Generator torque curve

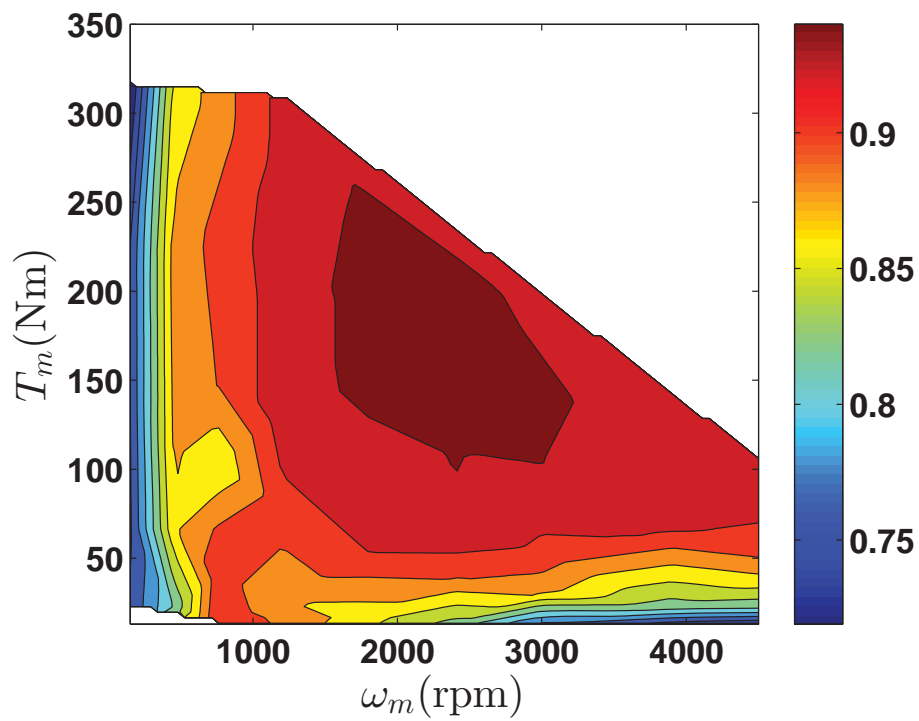

Figure 4.6: Motor efficiency surface (\%)

off at $2700 \mathrm{rpm}$ and $4000 \mathrm{rpm}$ for the motor and generator, respectively, which is in agreement with the evaluation report. The evaluation report efficiency curves showed high inefficiencies at low torque and speed and higher efficiencies at midrange to high torques and speeds, which again shows good correlation with the scaled 


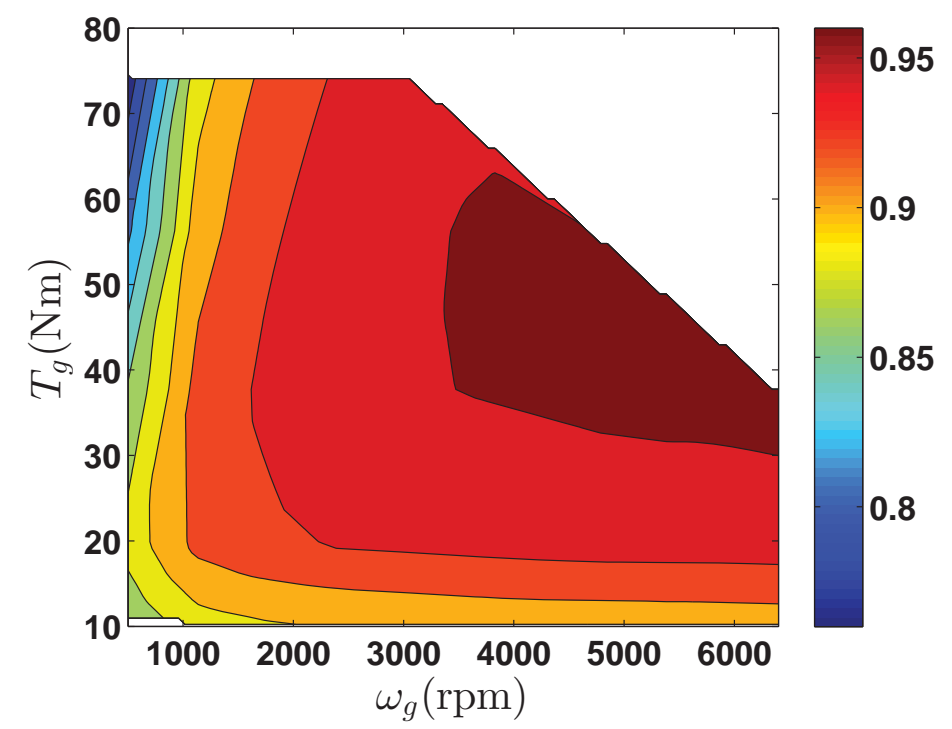

Figure 4.7: Generator efficiency surface (\%)

efficiency maps.

Lastly, the finally vehicle parameters used for this work can be found in Table 4.1 and were taken directly from the U.S. Department of Energy Prius evaluation report [80].

\subsection{Vehicle Model Implementation}

The vehicle model was implemented in series of MATLAB ${ }^{\circledR}$ functions that load the calibration file and sequentially performed the calculations of Equations 4.2, 4.3, and 4.12. The vehicle parameters, engine torque curve, engine fuel map, motor torque curve, generator torque curve, motor efficiency map and generator efficiency map, shown respectively in Table 4.1 and Figures 4.2, 4.3, 4.4, 4.5, 4.6, and 4.7, were integrated into the calibration file, which was a single data structure. The actual code can be found in Appendix A. 
Table 4.1

Vehicle parameters

\begin{tabular}{|c|c|c|}
\hline Parameter & Units & Value \\
\hline$S$ & $N o$. & 30 \\
\hline$R$ & $N o$. & 78 \\
\hline$K$ & $N o$. & 3.91 \\
\hline$m$ & $k g$ & 1254 \\
\hline$I_{r}$ & $k g / m^{2}$ & 0.01 \\
\hline$I_{c}$ & $k g / m^{2}$ & 0.01 \\
\hline$I_{e}$ & $k g / m^{2}$ & 0.180 \\
\hline$I_{s}$ & $k g / m^{2}$ & 0.02 \\
\hline$I_{m}$ & $k g / m^{2}$ & 0.05 \\
\hline$I_{g}$ & $k g / m^{2}$ & 0.05 \\
\hline$r_{t i r e}$ & $m$ & 0.287 \\
\hline$\mu_{r}$ & $N D$ & 0.015 \\
\hline$a$ & $m^{2}$ & 2.25 \\
\hline$c_{d}$ & $N o$. & 0.3 \\
\hline$V_{o c}$ & $V$ & 201.6 \\
\hline$R_{b a t t}$ & $\Omega$ & 0.5 \\
\hline$C_{\text {batt }}$ & $A h r$ & 6.5 \\
\hline
\end{tabular}




\section{Chapter 5}

\section{Basis Function $S O C$ Optimization ${ }^{1}$}

This chapter will detail the initial research with a simplified vehicle model and a single input optimization. More specifically, this chapter describes a method to determine the fuel-optimal $S O C$ given any mobility drive cycle by exploiting the structure of the vehicle model of Equations 5.2, 5.3, and 5.4. These equations have three states, motor speed, $\omega_{m}$, engine speed, $\omega_{e}$ and battery state of charge, $S O C$, and three inputs, generator torque, $T_{g}$, engine torque, $T_{e}$, and motor torque, $T_{m}$. The fuel consumption is a function of both the state engine speed, $\omega_{e}$, and the engine torque, $T_{e}$. Given a mobility drive cycle and a $S O C$ time history, the model can be inverted and the torques computed uniquely. Once this is done, the fuel consumption can be computed using the engine performance curves of the previous chapter. To make the fuel consumption minimal, an optimal $S O C$ time history must be found and is described below.

This work consists of a two step, off line optimization method to determine the fuel optimal SOC profile for a complex drive cycle summarized in Figure 5.1. It can be used for any duty cycle, including propulsion power, electric power for government

${ }^{1}$ Reprinted with permission from [3] C) Inderscience Publishers. Letter of permission found in Appendix F 
furnished equipment (GFE), silent watch capability, and vehicle-to-grid (V2G) mobile energy exchange and storage, however, this analysis will only cover the propulsion cycle.

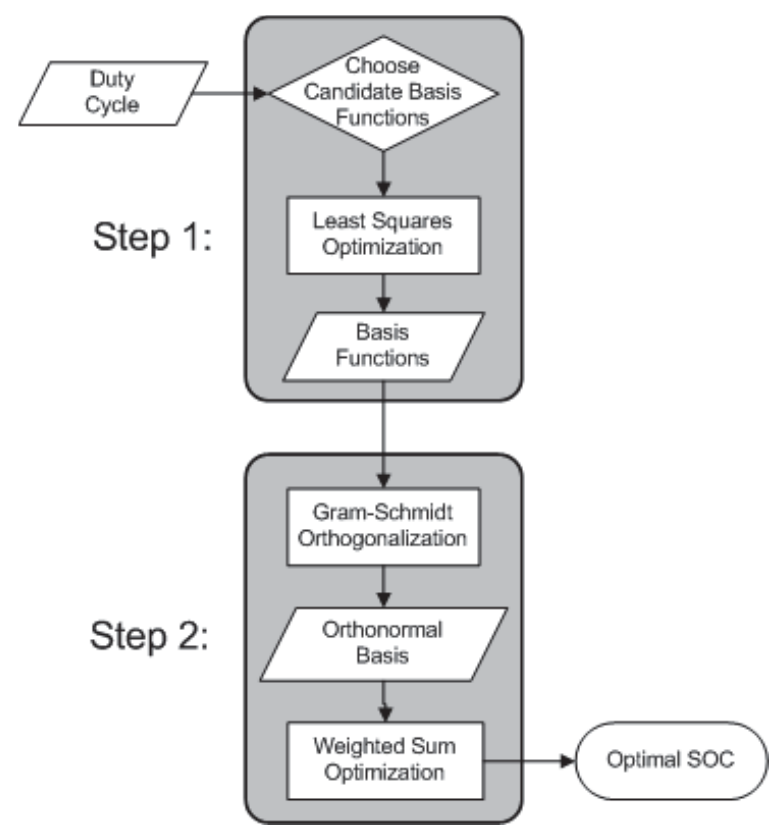

Figure 5.1: Two step optimization overview

This method is based on the assumption that the optimal $S O C$ is a function of the duty cycle, which was shown in [3]. Step one of the process, shown in Figure 5.1, decomposes the drive cycle into a series of orthogonal functions and uses a least squares nonlinear regression to determine the optimal frequencies based on the residual error. In step two, the series of periodic functions are transformed into an orthonormal basis and each of the vectors is weighted.

These weights or amplitudes are selected to minimize fuel usage. The normality of the vectors makes developing a solution in the feasible range a challenge and $S O C$ constraints are implemented as cost function penalty terms. 


\subsection{Fuel optimal $S O C$ problem definition}

Using a simplified version of the vehicle model, the desired $S O C$ profile can be found by solving the following optimization problem:

$$
\begin{gathered}
\text { minimize: } J=\sum_{t=t_{0}}^{t_{f}} \dot{m}_{f u e l}(S \dot{O} C) \\
\text { subject to: } S O C_{\min } \leq S O C \leq S O C_{\max } \\
t_{0} \leq t \leq t_{f}
\end{gathered}
$$

The fuel rate, $\dot{m}_{f u e l}$, determination is shown in Figure 5.2. By assuming a simplified torque loss term and a rule based engine speed, $\omega_{e}$, control shown in Table 5.1, a state space vehicle model can be constructed.

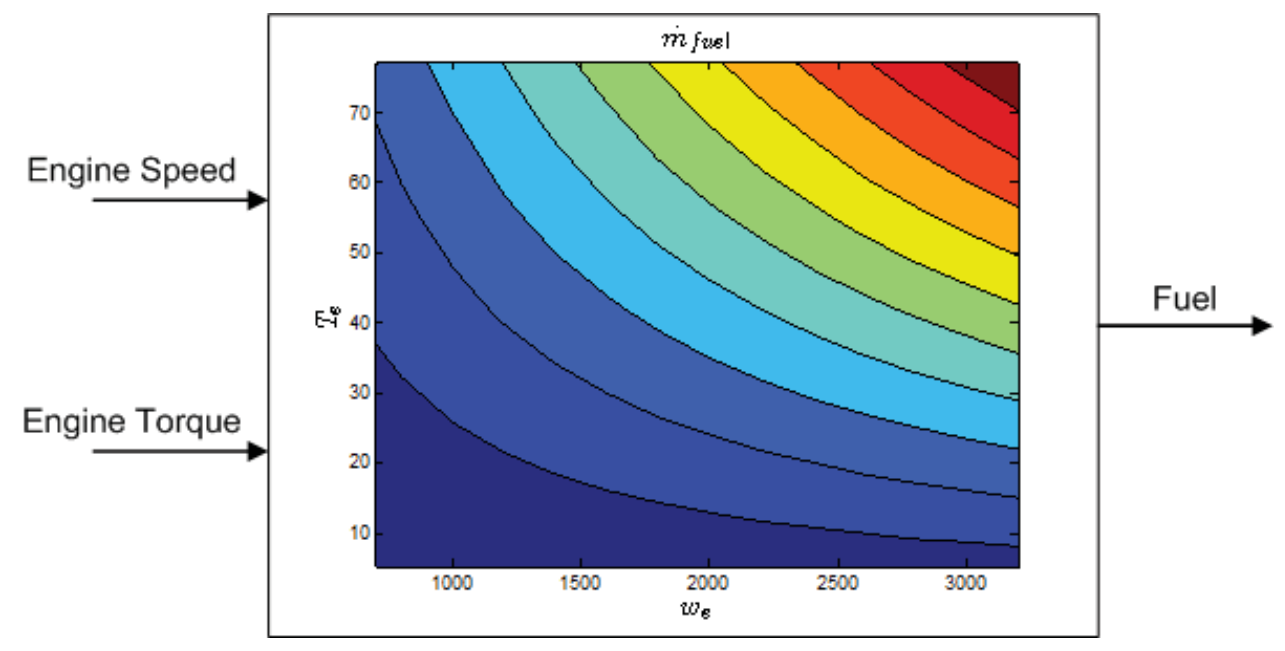

Figure 5.2: Map for relating engine speed and engine torque to fuel consumption.

Recall the vehicle model equations shown in Chapter 4, 


$$
\begin{gathered}
{\left[\frac{I_{v}^{\prime}(R+S)^{2}}{I_{e}^{\prime} K R}+\frac{I_{v}^{\prime} S^{2}}{I_{g}^{\prime} K R}+R\right] \dot{\omega}_{m}(t)=} \\
T_{m}(t)\left[\frac{(R+S)^{2}}{I_{e}^{\prime} R}+\frac{S^{2}}{I_{g}^{\prime} R}\right]+T_{e}(t)\left[\frac{(R+S)}{I_{e}^{\prime}}\right]+T_{g}(t)\left[\frac{S}{I_{g}^{\prime}}\right]-C\left[\frac{S^{2}}{I_{g}^{\prime} K R}+\frac{(R+S)^{2}}{I_{e}^{\prime} K R}\right] \\
{\left[(R+S)+\frac{I_{e}^{\prime} S^{2}}{I_{g}^{\prime}(R+S)}+\frac{I_{e}^{\prime} K R^{2}}{I_{v}^{\prime}(R+S)}\right] \dot{\omega}_{e}(t)=} \\
T_{m}(t)\left[\frac{K R}{I_{v}^{\prime}}\right]+T_{e}(t)\left[\frac{S^{2}}{I_{g}^{\prime}(R+S)}+\frac{K R^{2}}{I_{v}^{\prime}(R+S)}\right]-T_{g}(t)\left[\frac{S}{I_{g}^{\prime}}\right]-C\left[\frac{R}{I_{v}^{\prime}}\right] \\
S \dot{O} C(t)=\frac{-V_{o c}+\sqrt{V_{o c}^{2}-4 R_{\text {batt }}\left(T_{m} \omega_{m} \eta_{m}-T_{g} \omega_{g} \eta_{g}+E_{p w r}\right)}}{2 R_{b a t t} C_{b a t t}}
\end{gathered}
$$

Which would have to be linearized to create a typical state space representation. However, by treating $\dot{\omega}_{m}, \dot{\omega}_{e}$ and $S \dot{O} C$ as knowns in the system, the following transformation can be realized:

$$
\text { Let: } \overrightarrow{\dot{x}}=\left[\begin{array}{c}
\dot{\omega}_{m} \\
\dot{\omega}_{e} \\
S \dot{O} C
\end{array}\right] \text { and } \vec{u}=\left[\begin{array}{c}
T_{e} \\
T_{m} \\
T_{g}
\end{array}\right]
$$

Then, from Equations (4.2), (4.3) and (4.12), it can be stated that $\overrightarrow{\dot{x}}$ is a function of motor speed, $\omega_{m}$, engine speed, $\omega_{e}$, state of charge, $S O C$, engine torque, $T_{e}$, motor torque, $T_{m}$, and generator torque, $T_{g}$, over time:

$$
\dot{\vec{x}}=\vec{f}(\vec{x}, \vec{u}, t)
$$


Table 5.1

Rule based engine speed $\left(\omega_{e}\right)$ control

\begin{tabular}{|c|c|c|}
\hline Rule No. & $\begin{array}{c}\text { Vehicle Speed } \\
\omega_{m}(\mathrm{rpm})\end{array}$ & $\begin{array}{c}\text { Engine Speed } \\
\omega_{e}(\mathrm{rpm})\end{array}$ \\
\hline 1 & $0-5$ & 500 \\
\hline 2 & $6-15$ & 900 \\
\hline 3 & $16-30$ & 1500 \\
\hline 4 & $30-50$ & 2500 \\
\hline
\end{tabular}

Which can be re-written in the following form:

$$
\dot{\vec{x}}=\vec{f}(\vec{x})+B \vec{u}
$$

Solving for $\vec{u}$ :

$$
\vec{u}=B^{-1}(\dot{\vec{x}}-\vec{f}(\vec{x}))
$$

Expressions for $\vec{f}(\vec{x})$ and $B$ can be found in the appendix B. Therefore, knowing the motor speed, engine speed and state of charge over time, the torques can be determined and the fuel consumption can be calculated.

The inputs were determined in the following manner. First, the U.S. Army urban drive cycle shown in Figure 5.3, detailed in [61] was used as the propulsion cycle. The vehicle speed, $v$, was used to determine $\omega_{m}$ via Equation (5.9). Second, the engine speed, $\omega_{e}$, was determined via rule based control shown in Table 5.1. Last, Equation (5.1) and the fmincon [84] in MATLAB ${ }^{\circledR}$ was utilized to calculate the third input, $S O C$.

$$
\omega_{m}=\frac{v}{2 \pi r_{\text {tire }}}
$$




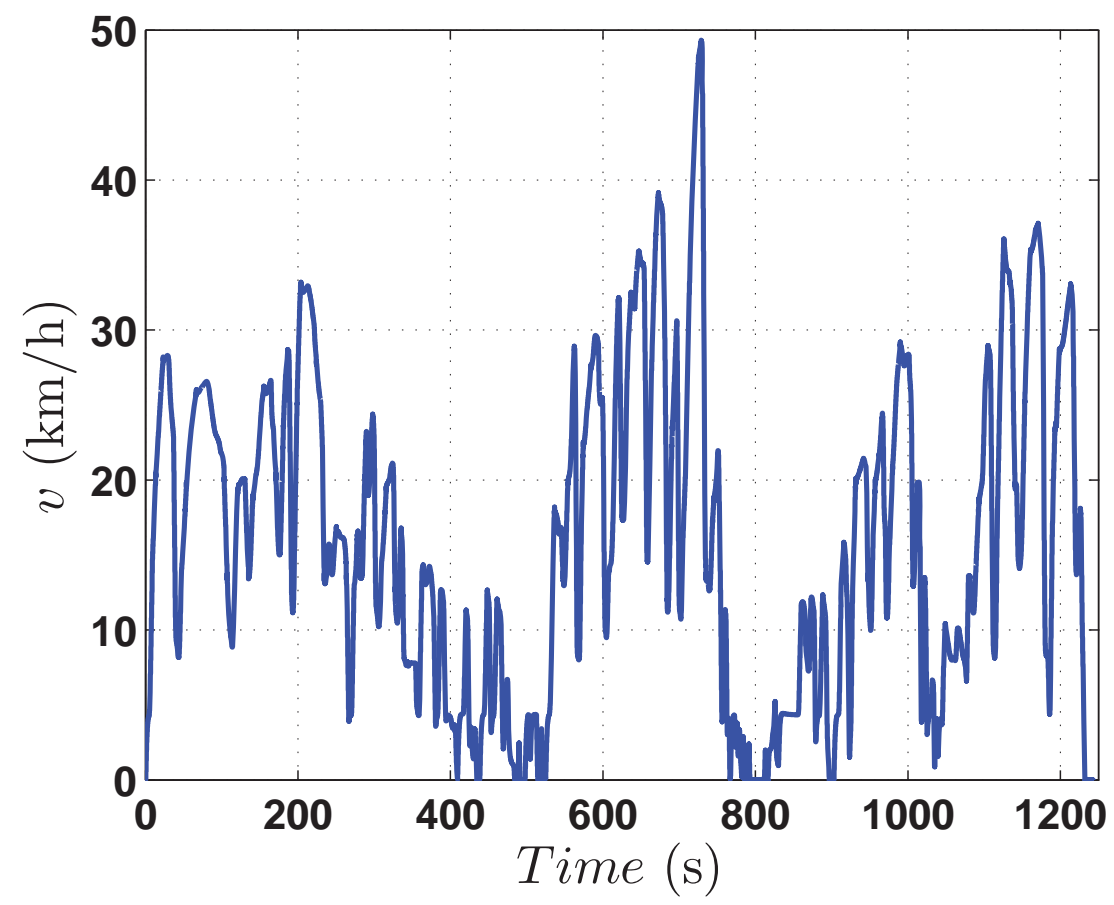

Figure 5.3: Military duty cycle - urban assault

As previously stated, Rizzo and Parker [3] used a simplified proof of concept drive cycle to illustrate that the optimal $S O C$ was directly related to the accelerations and decelerations of the drive cycle. This is intuitive from a physical stand point; it is most efficient to increase the $S O C$ when the vehicle is decelerating and decrease the $S O C$ when the vehicle is accelerating. Therefore, the characteristic behavior of the drive cycle will be used to guide the functional representation of $S O C$.

\subsection{Step 1: Drive Cycle Decomposition}

To determine the optimal $S O C$ time history, it is vital to understand the drive cycle characteristic components. This is because the optimal $S O C$ is a function of the drive cycle. Which characteristic components of the drive cycle effect the $S O C$ and how they effect the $S O C$ level is unknown, however. By visual inspection it is apparent 
Table 5.2

Cumulative residual error

\begin{tabular}{|c|c|}
\hline $\mathrm{n}$ & Error \\
\hline 2 & 8.6987 \\
\hline 3 & 7.4406 \\
\hline 4 & 7.4310 \\
\hline 5 & 7.0967 \\
\hline 6 & 6.9297 \\
\hline 7 & 6.9202 \\
\hline
\end{tabular}

that the drive cycle could be represented by a series of periodic functions such as Fourier series [85]. The general form of a Fourier series, which is series cosine and sine terms that represent a general periodic function, shown in (5.10).

$$
y(t)=a_{0}+\sum_{i=1}^{n}\left(a_{n} \cos n t+b_{n} \sin n t\right)
$$

To apply the Fourier transform and capture the frequency content down to $5 \mathrm{~Hz}$ of Figure 5.3, $n$ would have to equal 1250/.2 =6250, resulting in a large-scale optimization problem. Instead, a finite number of terms from the expansion of Equation (5.11) was used to estimate the drive cycle by identifying its dominate frequency components.

$$
\tilde{v}(t)=1+\sum_{i=1}^{n}\left(\cos 2 \pi f_{i} t+\sin 2 \pi f_{i} t\right)
$$

To determine $n$, Table 5.2, which summarized the cumulative residual error for each value of $n$ was created. It was determined the $n=6$ was appropriate from a error and manageability perspective.

Based on the method of least squares approximation [85] and considering the 1250 
samples of Figure 5.3, define the drive cycle approximation as:

$$
\tilde{v}=G(t) a
$$

Where:

$$
\tilde{v}=\left[\begin{array}{llll}
\tilde{v}\left(t_{1}\right) & \tilde{v}\left(t_{2}\right) \ldots \tilde{v}\left(t_{n}\right)
\end{array}\right]
$$

$G(t)=$

$$
\left[\begin{array}{cccccc}
1 & \cos f_{1} t_{1} & \sin f_{1} t_{1} & \cdots & \cos f_{6} t_{1} & \sin f_{6} t_{1} \\
1 & \cos f_{1} t_{2} & \sin f_{1} t_{2} & \cdots & \cos f_{6} t_{2} & \sin f_{6} t_{2} \\
\vdots & \vdots & \vdots & \cdots & \vdots & \vdots \\
1 & \cos f_{1} t_{n} & \sin f_{1} t_{n} & \cdots & \cos f_{6} t_{n} & \sin f_{6} t_{n}
\end{array}\right]
$$

And,

$$
\begin{aligned}
& a=\left(G^{T} G\right)^{-1} G^{T} v_{m} \\
& v_{m}=\left[\begin{array}{llll}
v_{m}\left(t_{1}\right) & v_{m}\left(t_{2}\right) & \ldots & v_{m}\left(t_{n}\right)
\end{array}\right]
\end{aligned}
$$

For a given set of frequencies $\left(f_{1}, f_{2}, \ldots f_{n}\right), \tilde{v}$ can be determined and the residual error from the measured vehicle speed, $v_{m}$, is calculated. Therefore, the first optimization problem can be constructed by finding $f_{i}$ that minimizes $\left\|v_{m}(t)-\tilde{v}(t)\right\|$, where $a$ are found to be the least square solution of Equation (5.12).

The results of the optimization problem are found in Figure 5.4, which is a plot of time $(\mathrm{s})$ versus vehicle speed $(\mathrm{km} / \mathrm{n})$. The important item to note is that not only is an approximation determined, but the decomposition of the cycle is now known. 
Therefore, the most important functions related to optimization of $S O C$ for fuel efficiency can be determined.

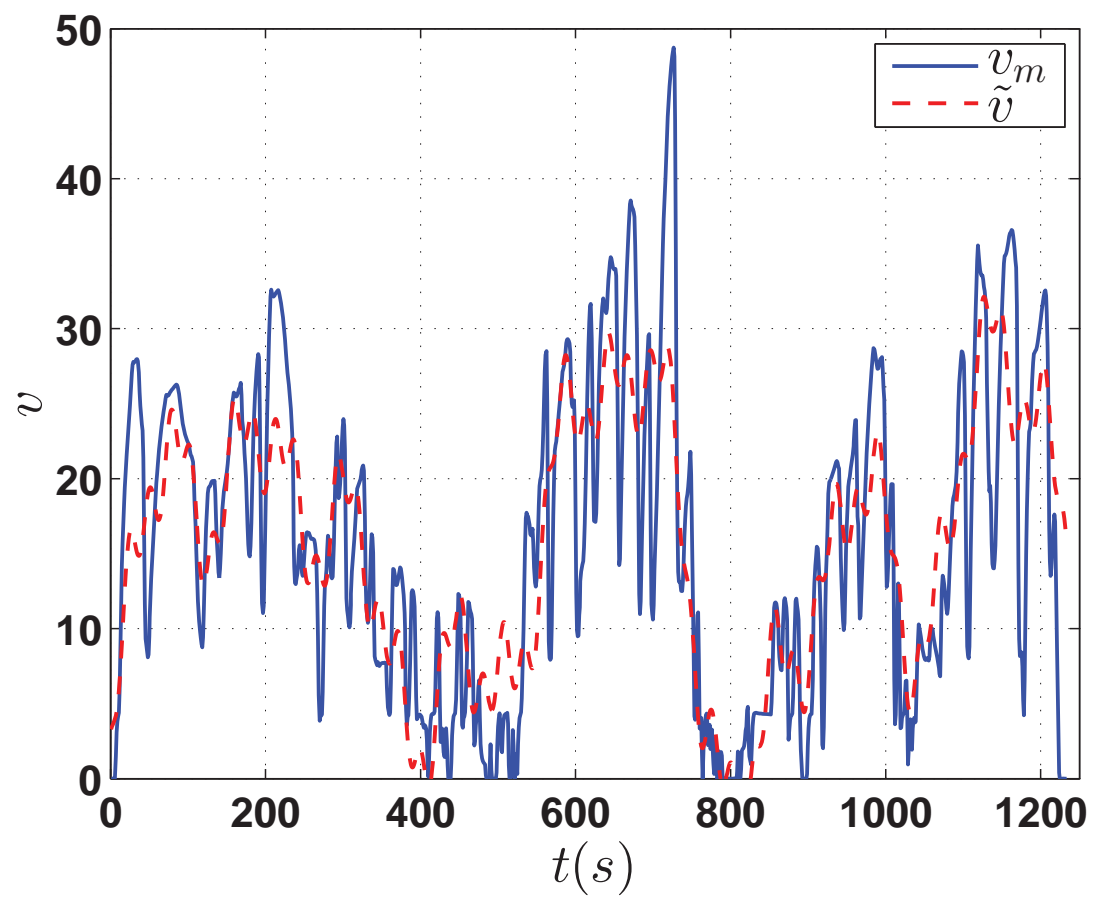

Figure 5.4: Measured $\left(v_{m}\right)$ vs. approximate $(\tilde{v})$ vehicle Speed

\subsection{Step 2: $S O C$ Optimization}

The next step is to compute the dominant terms of Equation (5.11) to optimize $S O C$. The first step is to ensure that the vectors are independent of each other. This is accomplished through the Gram-Schmidt orthogonalization procedure, detailed in Equation (5.17) [86].

$$
\hat{g}_{n}=a_{n}-\sum_{i=1}^{n-1}\left\langle g_{i}, a_{n}\right\rangle a_{n}
$$


In addition, the vectors are normalized (5.18) so that an orthonormal basis is created. Independence of the basis functions provides the possibility of gaining additional insight from the minimum fuel solution. In particular, identifying which terms have the most influence on fuel consumption.

$$
g_{n}=\frac{\hat{g}_{n}}{\left\|\hat{g}_{n}\right\|}
$$

Recall the optimization problem:

$$
\begin{gathered}
\text { minimize: } J=\sum_{t=t_{0}}^{t_{f}} \dot{m}_{f u e l}(S \dot{O} C) \\
\text { subject to: } S O C_{\min } \leq S O C \leq S O C_{\max } \\
t_{0} \leq t \leq t_{f}
\end{gathered}
$$

where:

$$
\begin{aligned}
S O C & =\sum_{i=1}^{n} w_{i} g_{i} \\
w_{i} & =\text { weight (Optimization Parameter) } \\
g_{i} & =\text { orthonormal basis }
\end{aligned}
$$

\subsection{Results}

To obtain a solution in the $S O C$ range of $S O C=25-100 \%$, two different methods were employed to understand the most effective solution and to explore the idea of local versus global minimum. Both methods included expanding the cost function so that the optimization would be penalized if the solution fell out of the feasible range. 
First, a baseline case needed to be determined. For this case, only one $S O C$ level was allowed and it showed that holding a constant $S O C$ over the input drive cycle used $16.58 \mathrm{~kg}$ of fuel.

The first cost function, shown in (5.19), penalized excursions above or below desired $S O C$ bounds.

$$
\begin{array}{r}
J_{1}=m_{\text {fuel }}+c_{1}\left(\frac{\min (S O C)-S O C_{\min }}{S O C_{\min }}\right)^{2}+ \\
c_{2}\left(\frac{\max (S O C)-S O C_{\max }}{S O C_{\max }}\right)^{2}
\end{array}
$$

The second cost function shown in Equation (5.20), applied a penalty for the number of points that were located outside the feasible $S O C$ range.

$$
\begin{array}{r}
J_{2}=m_{\text {fuel }}+c_{1}\left(\sum \mid \text { SOCPoints }<S O C_{\text {min }} \mid\right)+ \\
c_{2}\left(\sum \mid \text { SOCPoints }>S O C_{\text {max }} \mid\right)
\end{array}
$$

As with any numerical optimization problem, a good initial estimate is required and plays an important role. To address this problem, the fuel terms in Equations (5.19) and (5.20) were set to zero and the optimization solver was allowed to run until the solution was within the feasible range. The fuel term was then included for the final optimization and provided a feasible initial guess.

The optimal $S O C$ solutions are shown in Figure 5.5. This first cost function $S O C$ trajectory used $15.72 \mathrm{~kg}$ of fuel, a $5.1 \%$ improvement over the constant $S O C$ case. The second cost function required $15.64 \mathrm{~kg}$ of fuel, a $5.7 \%$ improvement over the constant 
$S O C$ case. This illustrated that the second cost function produced a smaller fuel usage for this particular example.

\subsection{Discussion}

Figure 5.5 shows that both curves exploit the lower frequency content from the drive cycle, but the second cost function utilizes the higher frequency content as well, which results in a further gain in fuel economy.

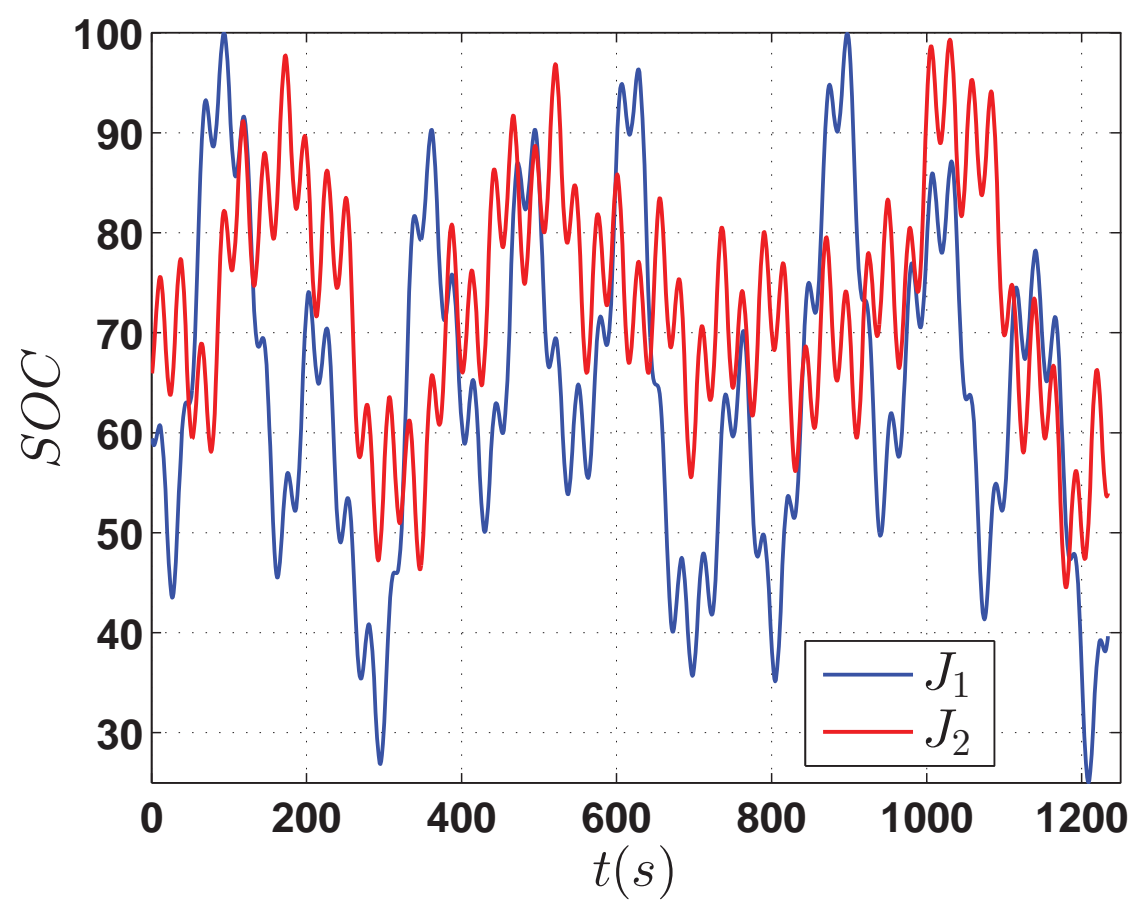

Figure 5.5: $S O C$ comparison

To further illustrate the importance of the higher frequencies, an optimal solution was obtained using the first three, lowest frequency terms in the $S O C$ expansion using $J 2$. This case resulted in a total fuel consumption of $16.10 \mathrm{~kg}$, which is expected due to the reduced frequency content. Figure 5.6 shows the three $S O C$ curves together and 
their respective fuel used. This further emphasizes the importance of high frequency content with respect to fuel usage.

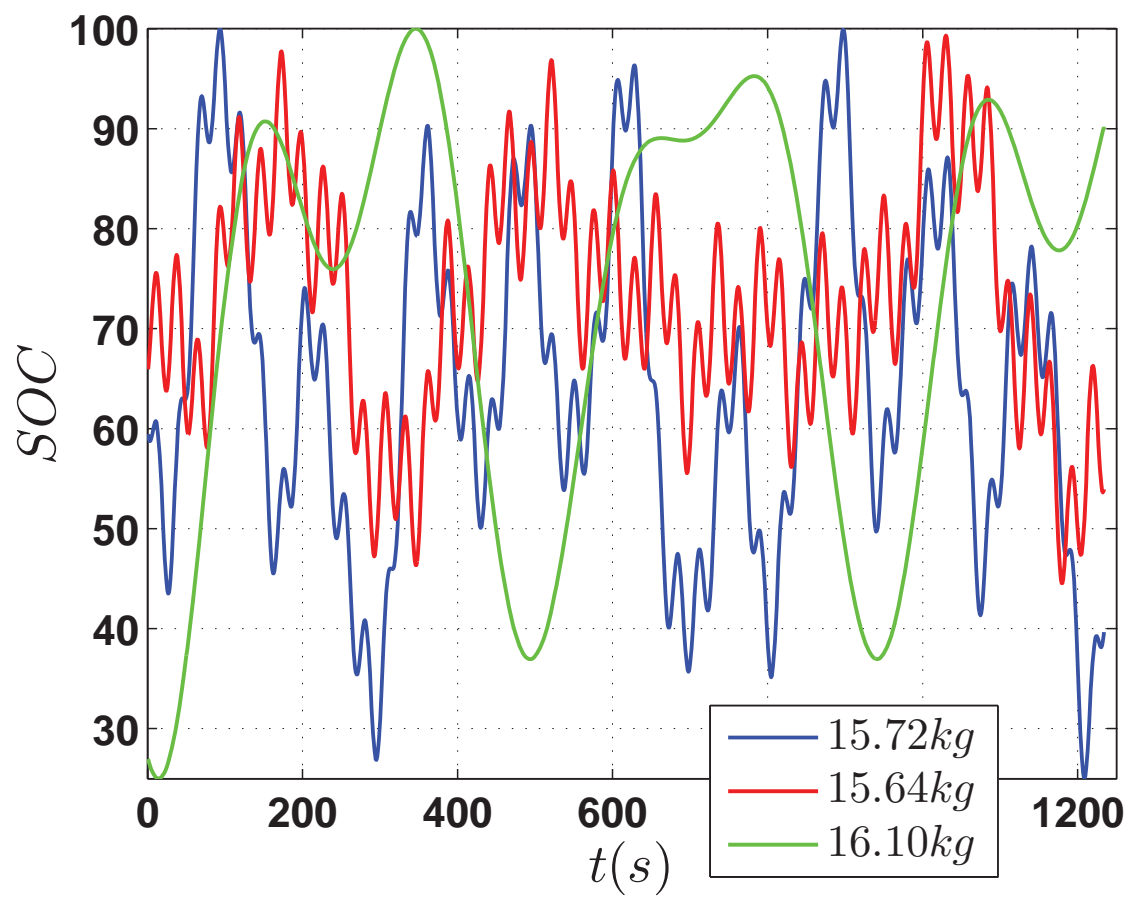

Figure 5.6: $S O C$ comparison with simplified model

An important implication of these results is the potential ability to be used in assisting in the vehicle components' design and selection. For example, a trade study could be conducted to understand the cost-benefit relationship of a faster responding, higher bandwidth system; for example, the component cost required to achieve the $5.7 \%$ improvement may be cost prohibitive. Another benefit is understanding the limitation of the system's open-loop control. Perhaps the high frequency behavior seen in Figure 5.5 cannot be achieved with a feed-forward control system, therefore more stringent requirements would be required for the feedback portion of the control system which again has a cost impact. 


\subsection{Summary}

This chapter detailed a two step optimization method consisting of decomposing the drive cycle into a series of periodic functions. The frequencies of the functions were determined by using a least squares regression and optimizing the estimated frequencies based on the residual error. This sequence of periodic functions was then transformed into an orthonormal basis. Each of the vectors of the basis were weighted and these weights or amplitudes were optimized based on fuel used.

The $S O C$ inequality constraints were achieved using a penalty terms applied to the minimum fuel cost function. These terms penalized the cost function for falling out of the usable range. Two different approaches were explored and yielded different minimum fuel solutions. The results of both methods concluded that the low frequency was the dominate feature to minimize $S O C$ and provided an $5.1 \%$ reduction in fuel consumption. However, the addition of the high frequency content provided $.6 \%$ further reduced fuel consumption.

The results provide valuable insight into the dependence on minimum fuel solutions to SOC management. However, this batch optimization approach is not suitable for real-time control due to its two-part process. Therefore, the next chapter will detail a multiple input optimization framework with a full vehicle model that can be used for controls and design. 


\section{Chapter 6}

\section{Multiple Input Optimization}

The results shown in Chapter 5 were significant because they explained how shaping the $S O C$ over time in an optimal way can minimize fuel consumption. In addition, this method could be used to compare hybrid vehicle designs to understand how each system could react to a particular drive cycle, which could be particularly useful to the U.S. Army when sizing vehicle components such as electric motors and batteries. However, the results would be limited in scope due to simplified vehicle model and rule based engine speed control. Additionally, the method depends on knowing the frequency content of the cycle and expansion was limited.

Building on the previous result that an optimal $S O C$ profile can minimize fuel use, a multiple input optimization problem was developed. One approach to generating the optimal profiles would be to numerically solve the two-point boundary value problem resulting from the necessary conditions of optimaility. Instead, a direct numerical method was used to determine the profiles' discretized levels. This method utilizes the complete vehicle model and takes into account the propulsion and electrical duty cycles as well as stationary microgrid requirements, making it useful for optimal controls development. In addition, the formulation can include vehicle parameters, which makes it extremely useful for vehicle design optimization. 


\subsection{Problem Formulation}

To minimize the energy use of a hybrid vehicle as described in Chapter 4, the optimization problem must focus on the fuel used by the combustion engine, since the engine generates the energy via the fuel. Therefore, the goal is to minimize the cost function of Equation 6.1

$$
J=\int_{t_{0}}^{t_{f}} \dot{m}_{f u e l} d t
$$

Fuel rate, $\dot{m}_{f u e l}$, is a function of engine speed, $\omega_{e}$, and engine torque, $T_{e}$, and calculated using a surface look-up as shown in Figure 7.7, which is a transformation of the brake specific fuel map, shown in Chapter 4 via Equation (6.2). By integrating the value from the table look-up at each time step over the simulation duration, the total fuel consumption can be calculated.

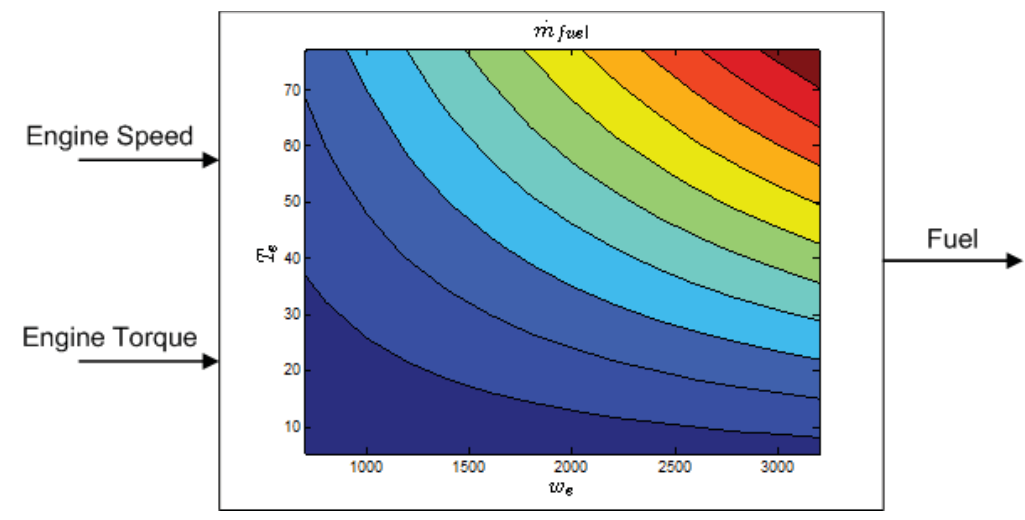

Figure 6.1: Map for relating engine speed $\left(\omega_{e}\right)$ and torque $\left(T_{e}\right)$ to fuel consumption.

$$
\dot{m}_{\text {fuel }}=B S F C * P
$$

The engine torque, $T_{e}$, is limited by the engine speed, $w_{e}$. Therefore, the optimization 
problem Equation (6.1) can be expanded as shown in Equation (6.3).

$$
\begin{aligned}
& \text { minimize: } J=\int_{t_{0}}^{t_{f}} \dot{m}_{f u e l}\left(\omega_{e}, T_{e}\right) d t \\
& \text { subject to: } 0 \leq T_{e} \leq T_{e_{\max }}\left(\omega_{e}\right)
\end{aligned}
$$

Recalling from Chapter 4, engine speed can be calculated from Equations (6.4) and (6.5) if the time histories of motor, engine and generator torques are known as well as the brake force.

$$
\dot{\omega}_{e}(t)=\frac{T_{m}(t)\left[\frac{K R}{I_{v}^{\prime}}\right]+T_{e}(t)\left[\frac{S^{2}}{I_{g}^{\prime}(R+S)}+\frac{K R^{2}}{I_{v}^{\prime}(R+S)}\right]-T_{g}(t)\left[\frac{S}{I_{g}^{\prime}}\right]-C\left[\frac{R}{I_{v}^{\prime}}\right]}{\left[(R+S)+\frac{I_{e}^{\prime} S^{2}}{I_{g}^{\prime}(R+S)}+\frac{I_{e}^{\prime} K R^{2}}{I_{v}^{\prime}(R+S)}\right]}
$$

where:

$$
\begin{aligned}
& I_{v}^{\prime}=I_{m} K+I_{r} K+\frac{m r_{t i r e}^{2}}{K} \\
& I_{g}^{\prime}=I_{s}+I_{g} \\
& I_{e}^{\prime}=I_{c}+I_{e} \\
& C=T_{f b}+r_{\text {tire }} m \mu_{r} g+\frac{0.5 C_{d} r_{t i r e}{ }^{3} a \rho \omega_{m}(t)^{2}}{K^{2}} \\
& \omega_{e}(t)=\int_{t_{o}}^{t_{f}} \dot{\omega}_{e}(\tau) d \tau
\end{aligned}
$$

The motor speed can be calculated directly from the vehicle speed, $v$, which is an input as described in Chapter 3, through Equation (6.6).

$$
\omega_{m}=\frac{v K}{2 \pi r_{\text {tire }}}
$$


The motor torque, $T_{m}$, and generator torque, $T_{g}$, are limited by the motor speed, $\omega_{m}$, and generator speed, $\omega_{g}$, respectively. The braking force, $T_{f b}$, is limited by design of the brake system. Therefore, the optimization problem can be further expanded as shown in Equation (6.3).

$$
\begin{array}{cl}
\text { minimize: } J=\int_{t=t_{0}}^{t_{f}} \dot{m}_{f u e l}\left(T_{e}, T_{g}, T_{f b}, T_{m}\right) d t \\
\text { subject to: } 0 \leq T_{e} \leq T_{e_{\max }}\left(\omega_{e}\right) \\
0 \leq T_{g} \leq T_{g_{\max }}\left(\omega_{g}\right) \\
T_{m_{\min }}\left(\omega_{m}\right) \leq T_{m} \leq T_{m_{\max }}\left(\omega_{m}\right) \\
0 \leq T_{f b} \leq T_{f b_{\max }}
\end{array}
$$

One of the degrees of freedom of the system was removed by requiring the vehicle to attain a desired vehicle speed profile shown in Figure 7.1. Since the motor torque Equation (6.8) is given in terms of motor acceleration, $\omega_{m}$, the desired vehicle speed can be transformed through Equation (6.9).

$$
\begin{gathered}
T_{m}(t)=\frac{\left[\frac{I_{v}^{\prime}(R+S)^{2}}{I_{e}^{\prime} K R}+\frac{I_{v}^{\prime} S^{2}}{I_{g}^{\prime} K R}+R\right] \dot{\omega}_{m}(t)-T_{e}(t)\left[\frac{(R+S)}{I_{e}^{\prime}}\right]-T_{g}(t)\left[\frac{S}{I_{g}^{\prime}}\right]+C\left[\frac{S^{2}}{I_{g}^{\prime} K R}+\frac{(R+S)^{2}}{I_{e}^{\prime} K R}\right]}{\left[\frac{(R+S)^{2}}{I_{e^{\prime}} R}+\frac{S^{2}}{I_{g}^{\prime} R}\right]} \\
\dot{\omega}_{m}(t)=\frac{d\left(\omega_{m}\right)}{d t}=\frac{d\left(\frac{v K}{2 \pi r_{\text {tire }}}\right)}{d t}
\end{gathered}
$$




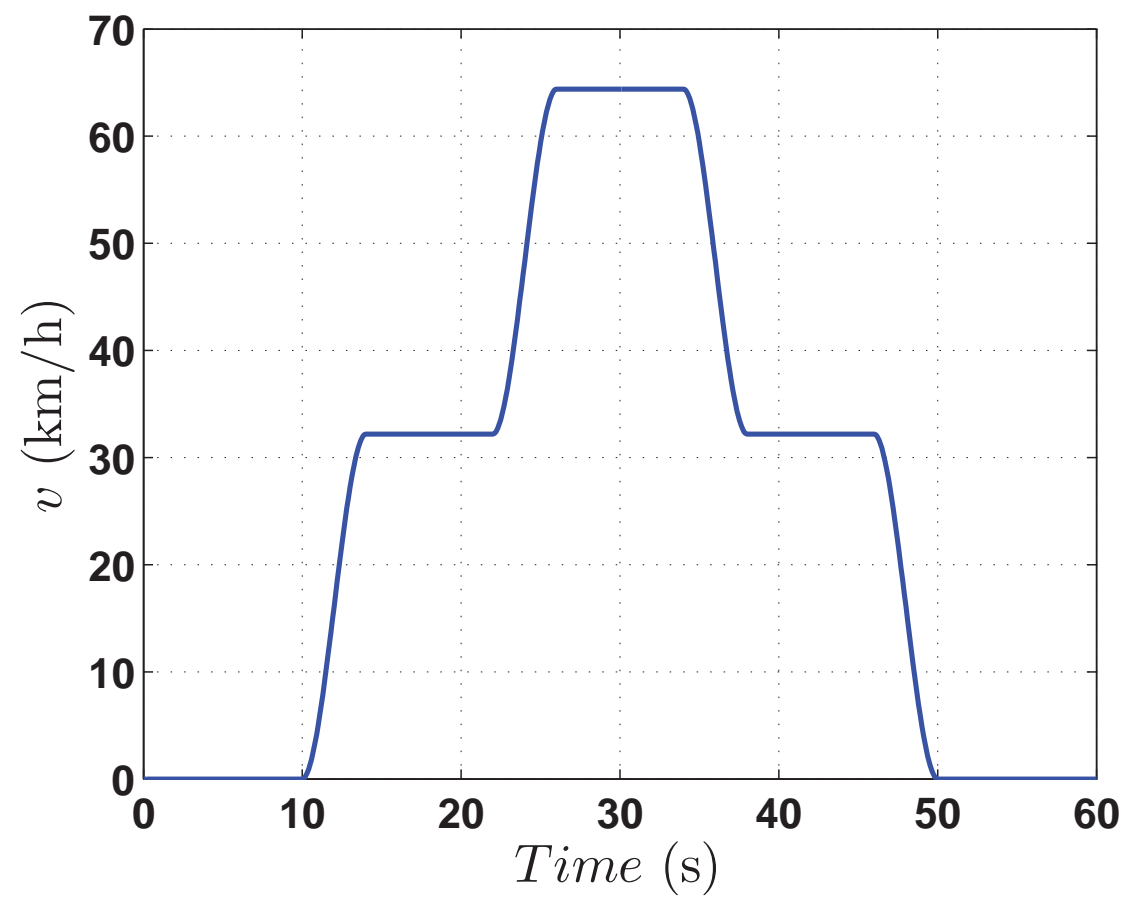

Figure 6.2: Propulsion duty cycle (originally shown in Chapter3)

Then, the optimization problem can be written as shown in Equation (6.10).

$$
\begin{aligned}
\text { minimize: } J=\int_{t=t_{0}}^{t_{f}} \dot{m}_{f u e l}\left(T_{e}, T_{g}, T_{f b}\right) d t \\
\text { subject to: } 0 \leq T_{e} \leq T_{e_{\max }}\left(\omega_{e}\right) \\
0 \leq T_{g} \leq T_{g_{\max }}\left(\omega_{g}\right) \\
T_{m_{\min }}\left(\omega_{m}\right) \leq T_{m} \leq T_{m_{\max }}\left(\omega_{m}\right) \\
0 \leq T_{f b} \leq T_{f b_{\max }}
\end{aligned}
$$

There is one final constraint that is vital in allowing the system to perform like a true hybrid vehicle and that is battery state of charge $(S O C)$. The $S O C$ of the system can be calculated using Equations (6.11) and (6.12) from Chapter 4. 


$$
\begin{gathered}
S \dot{O} C(t)=\frac{-V_{o c}+\sqrt{V_{o c}^{2}-4 R_{b a t t}\left(T_{m} \omega_{m} \eta_{m}-T_{g} \omega_{g} \eta_{g}+E_{p w r}\right)}}{2 R_{b a t t} C_{b a t t}} \\
S O C=\int_{t_{o}}^{t_{f}} S \dot{O} C(t) d t
\end{gathered}
$$

where $E_{\text {pwr }}$ is an input described in Chapter 3 and generator speed, $\omega_{g}$ is determined by the kinematic relationship between the generator, engine and motor, described by Equation 6.13.

$$
\omega_{g}=\frac{\omega_{e}(R+S)-\omega_{m} R}{S}
$$

Finally, the optimization problem can be written as find $T_{e}, T_{g}$, and $T_{f b}$ that:

$$
\begin{array}{cl}
\text { minimizes: } J=\int_{t=t_{0}}^{t_{f}} \dot{m}_{f u e l}\left(T_{e}, T_{g}, T_{f b}\right) d t \\
\text { subject to: } 0 \leq T_{e} \leq T_{e_{\max }}\left(\omega_{e}\right) \\
0 \leq T_{g} \leq T_{g_{\max }}\left(\omega_{g}\right) \\
T_{m_{\min }}\left(\omega_{m}\right) \leq T_{m} \leq T_{m_{\max }}\left(\omega_{m}\right) \\
0 \leq T_{f b} \leq T_{f b_{\max }} \\
S O C_{\min } \leq S O C \leq S O C_{\max } .
\end{array}
$$

The optimization problem of Equation 6.14 was combined with the vehicle simulation of Chapter 4 and solved using MATLAB's ${ }^{\circledR}$ fmincon function [84].The calculation order is shown in Figure 6.3 and the actual code can be found in Appendix A. 


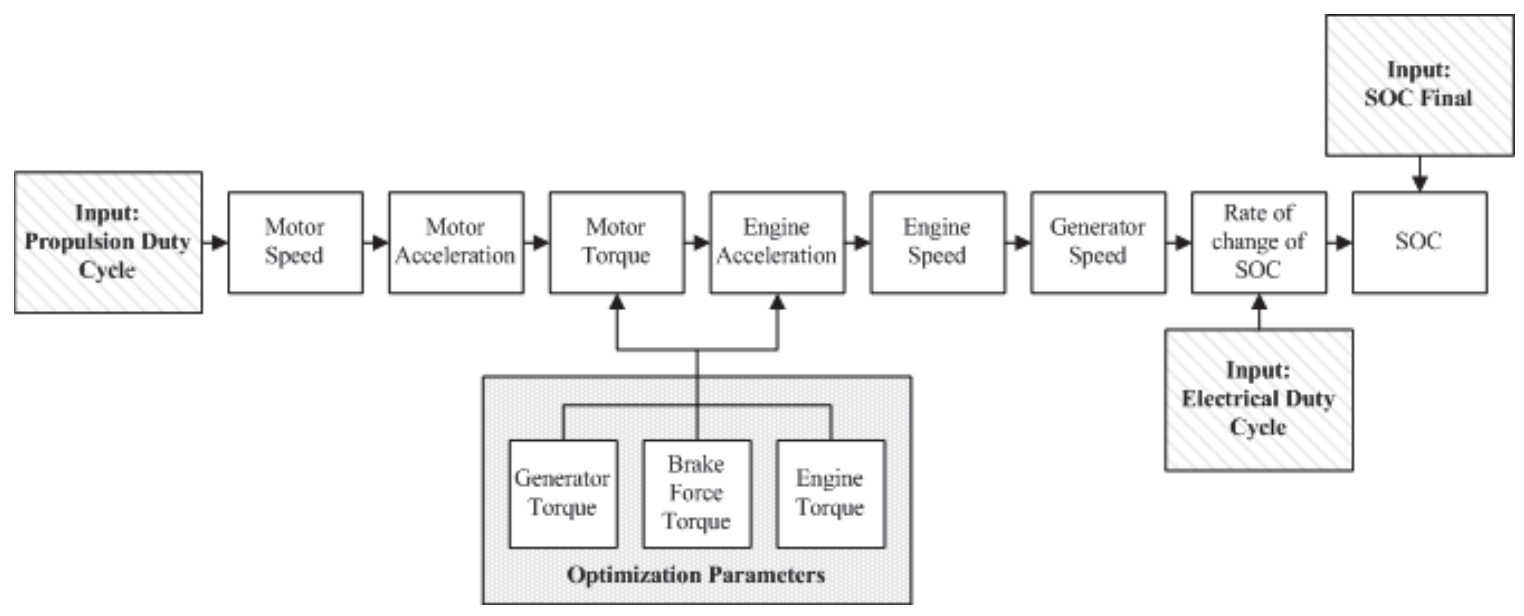

Figure 6.3: Calculation order

\subsection{Constraints}

Formulation of the constraints plays a large role in the optimization problem. This section will detail the practical development of the constraints. All of the constraints were developed as equality constraints set equal to zero as described by Equation (6.15) and (6.16). For Equation, (6.15), the first equation is used for the maximum condition and the second is used for the minimum condition.

$$
\begin{aligned}
& c_{n}=-x_{n}+x_{n_{\max }} \text { or } c_{n}=x_{n}-x_{n_{\min }} \\
& c_{e q}=\sum\{y \mid y \subset c, y<0\} \stackrel{!}{=} 0
\end{aligned}
$$

The values for the minimum and maximum thresholds were chosen in the following manner. For $T_{m_{\max }}, T_{e_{\max }}$ and $T_{g_{\max }}$, their respective torque curves were used to determine their maximum value as a function of their current speed. For $T_{m_{m i n}}$, the negative of the torque curve was used to determine the minimum value as a function of the motor's speed. The brake force torque, $T_{f b_{\max }}$, which is a constant value determined by vehicle design, was set to zero when the vehicle was accelerating, cruising at steady state or stationary. $S O C_{\max }$ was set to $100 \%$, with the exception of 
$S O C_{\text {final }}$, which was set to the stationary grid requirement. $S O C_{\min }$ was set to $30 \%$, with the exception of $S O C_{\text {final }}$, which was set to the stationary grid requirement.

\subsection{Numerical Integration Considerations}

The optimization degrees of freedom are the discretized amplitude of $T_{e}, T_{g}$, and $T_{f b}$. A $1 \mathrm{~Hz}$ discretization was used resulting in 183 degrees of freedom. There are two integrations that take place in the calculation and they are used to determine engine speed, $\omega_{e}$, and battery state of charge, $S O C$. Therefore, an integration method and an appropriate time step was required. The trajectory discretization was set to $1 \mathrm{~Hz}$, but it was not clear if this was adequate for integration, where large errors can build over time. Therefore, a known cycle, show in Figure 6.4 [61], which is considered to have the worst case for frequency content, was used to determine the integration method and step size was using Euler integration shown in Equation 6.17.

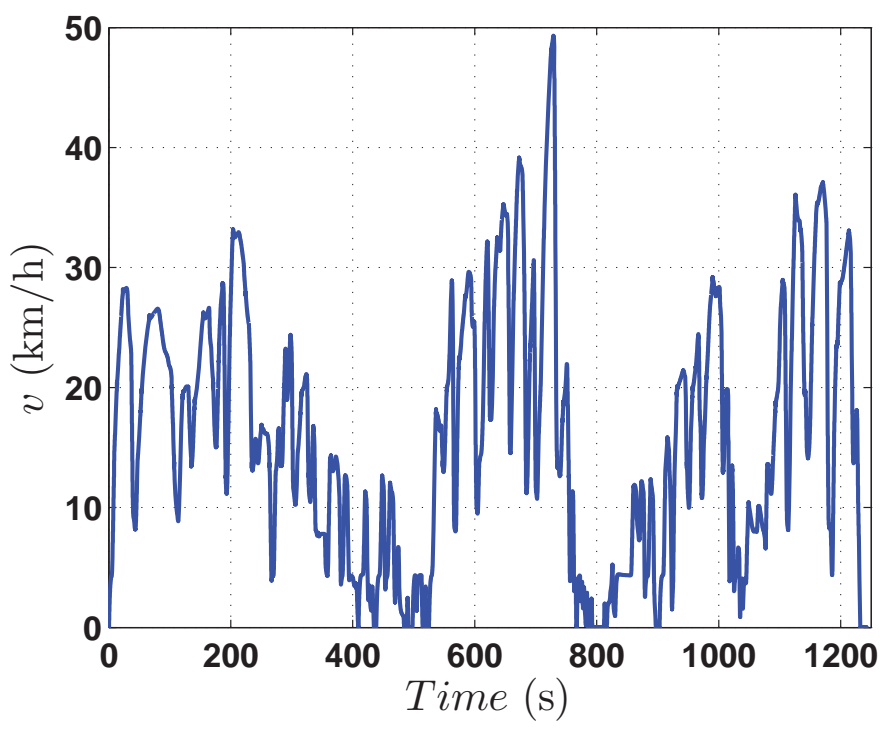

Figure 6.4: Urban assault cycle

The next step was to determine if Euler's method with a reasonable $d t$, could produced 
adequate results.

$$
\dot{\vec{x}}(t)=\vec{f}(\vec{x}, \vec{u}, t) \rightarrow \vec{x}_{n+1}=\vec{x}_{n}+\Delta t \vec{f}\left(\vec{x}_{n}, \vec{u}_{n}, t\right)
$$

The data was differentiated at the $1000 \mathrm{~Hz}, 100 \mathrm{H} \mathrm{Hz}, 10 \mathrm{~Hz}$ and $1 \mathrm{~Hz}$. Figure 6.5 shows the results for $d t=1 \mathrm{~s}$, which was too large and resulted in large errors. Figure 6.6 shows the result for $d t=.1 \mathrm{~s}$, which was chosen to be sufficient. Therefore, Euler's method with a $d t=.1$ was used for the analysis. The simulation code can be found in Appendix A.

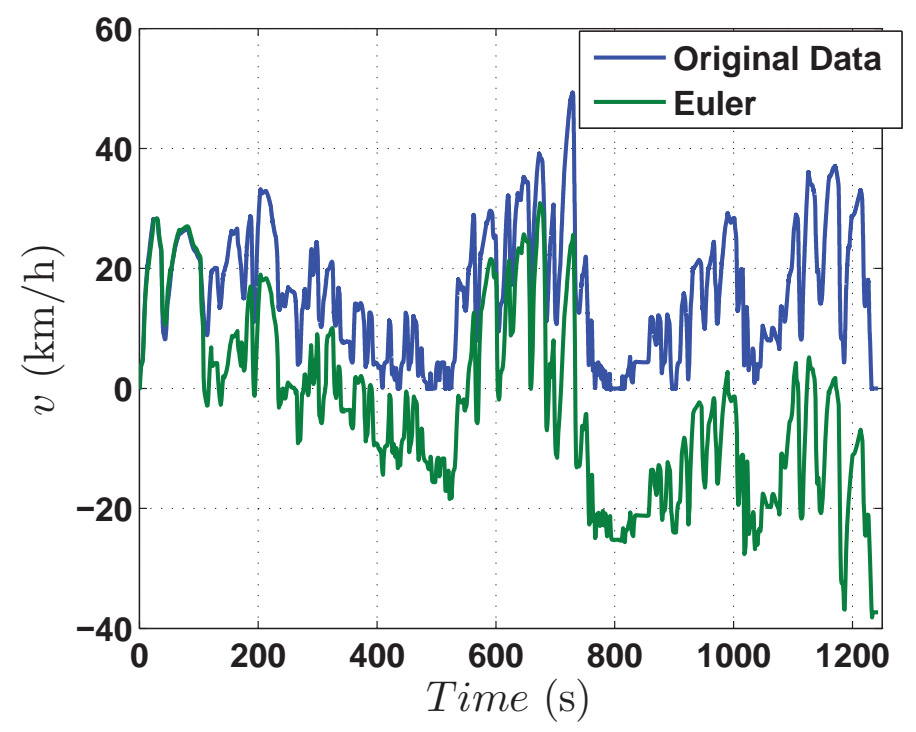

Figure 6.5: Integration with Euler's method and $d t=1 \mathrm{~s}$ 


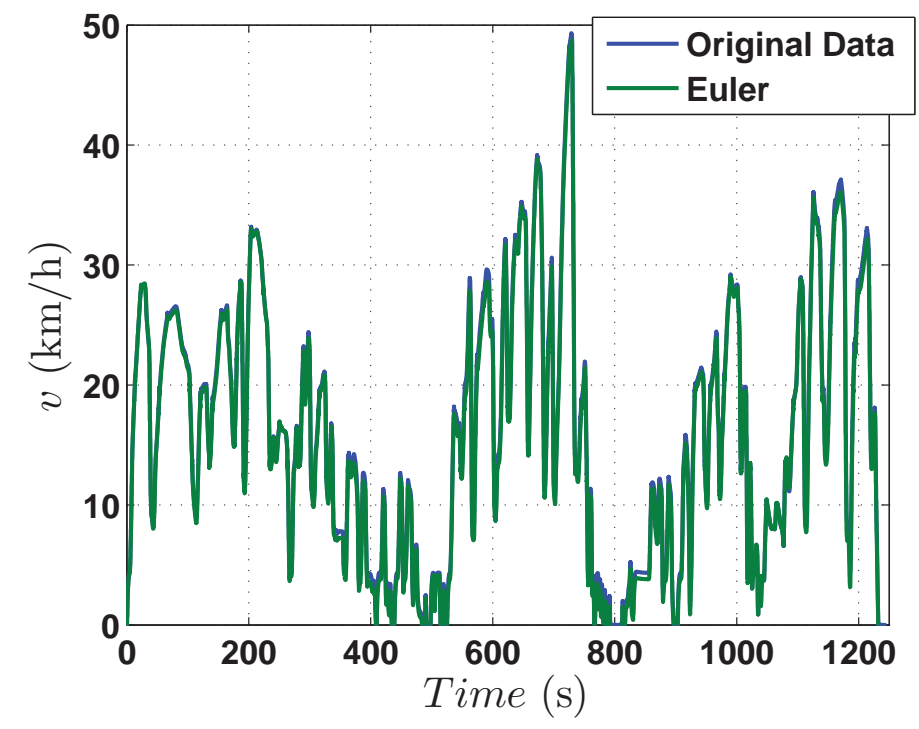

Figure 6.6: Integration with Euler's method and $d t=.1 \mathrm{~s}$

\subsection{Final Description of Numerical Optimization}

\section{Problem}

Now that the numerical optimization problem has been designed, the cost function can be expanded to include vehicle parameters to understand the trade off between fuel consumption and vehicle subsystem design. This could be especially useful in choosing the battery capacity or motors. By using the complete duty cycle detailed in Chapter 3, the effect of duty cycle choice on the vehicle design can also be shown. 
The optimization problem can now be stated as find $T_{e}, T_{g}$, and $T_{f b}$ that:

$$
\begin{array}{ll}
\text { minimizes: } J=w_{1} \sum_{t=t_{0}}^{t_{f}} \dot{m}_{f u e l}\left(T_{e}, T_{g}, T_{f b}\right)+w_{2}\left(\text { veh }_{\text {param }}\right) \\
\text { subject to: } 0 \leq T_{e} \leq T_{e_{\max }}\left(\omega_{e}\right) \\
0 \leq T_{g} \leq T_{g_{\max }}\left(\omega_{g}\right) \\
T_{m_{\min }}\left(\omega_{m}\right) \leq T_{m} \leq T_{m_{\max }}\left(\omega_{m}\right) \\
0 \leq T_{f b} \leq T_{f b_{\max }} \\
& S O C_{\min } \leq S O C \leq S O C_{\text {max }} \\
& \text { veh }_{\text {param }} \leq \text { veh }_{\text {maram }} \leq \text { veh }_{\text {param }}{ }_{\text {max }}
\end{array}
$$

where $w_{1}, w_{2}$ are weighing factors to shift cost emphasis between fuel and some positive function of the design parameters.

An example using battery capacity, $C_{b a t t}$, will be shown in the results section. 



\section{Chapter 7}

\section{Results}

\subsection{Power System Control}

Traditionally, closed loop proportional controllers are used manage unexpected disturbances in system control, however, they are not always efficient from a fuel consumption perspective. Therefore, this section will detail the results of using the numerical optimization to manage an unexpected electrical pulse load versus a proportional controller.

\subsubsection{Problem Statement}

The objective is to compare the fuel used by the numerical optimization method versus a proportional controller, which is detailed in the next section, during a relevant military mission. The real world scenario is a military hybrid vehicle on prescribed 
mission, shown in Figure 7.1, with a nominal electrical load of $300 \mathrm{~W}$, shown in Figure 7.2. A large unexpected $600 \mathrm{~W}$ pulse electrical load is required for a brief period of time, shown in Figure 7.2. Lastly, the stationary microgrid is requesting that the vehicle end the maneuver with a $S O C_{\text {final }}$ equal to $50 \%$.

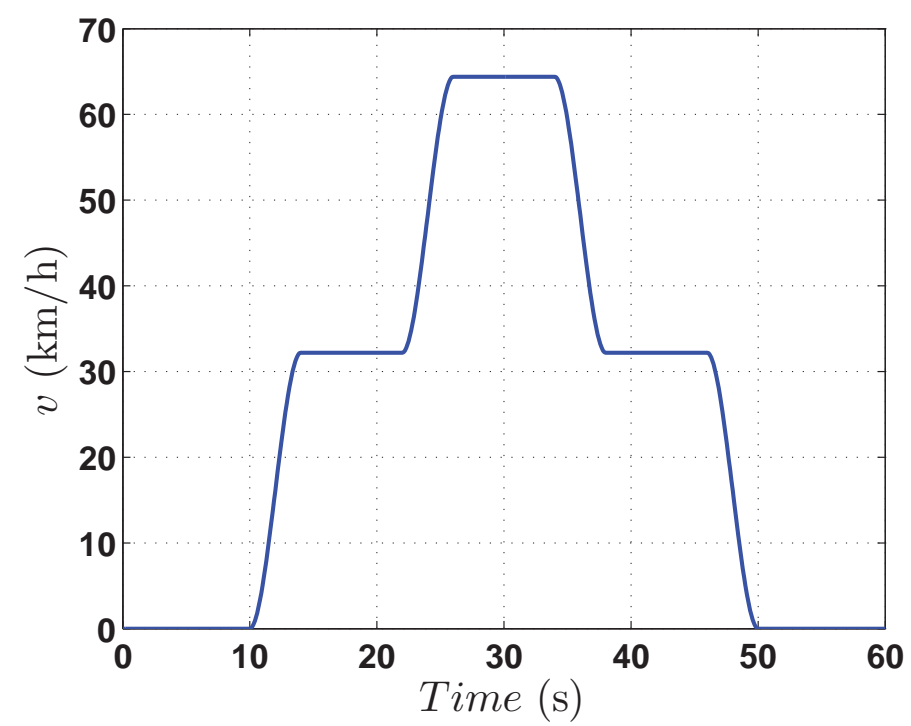

Figure 7.1: Propulsion duty cycle (originally shown in Chapter3)

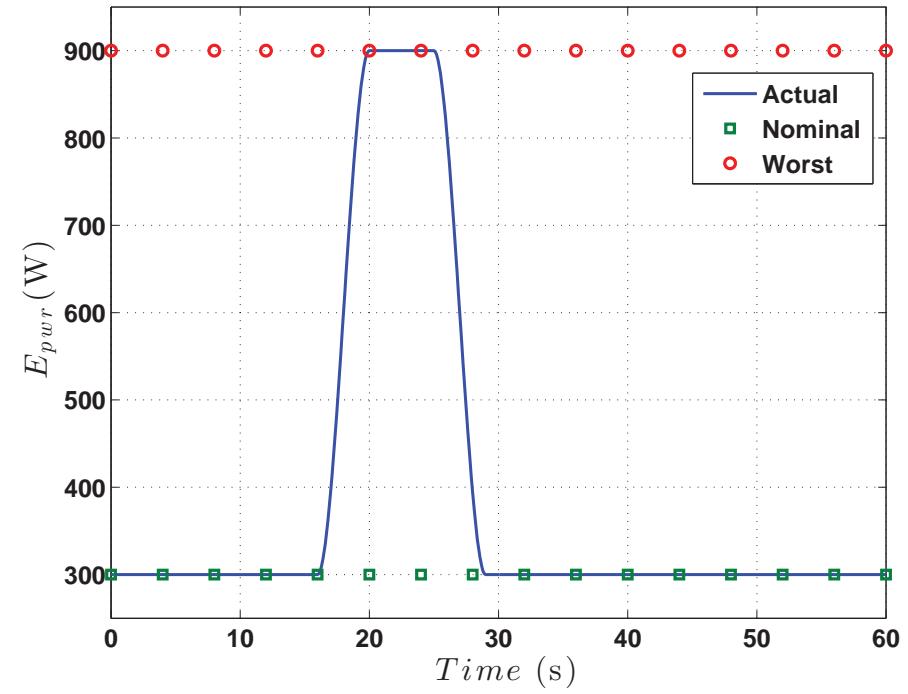

Figure 7.2: Electrical duty cycle (originally shown in Chapter3) 
The propulsion cycle and nominal electrical load (constant $300 \mathrm{~W}$ ) are known. The numerical optimization solver was discretized at $1 \mathrm{~Hz}$ over sixty seconds to determine optimal torque trajectories for the propulsion cycle and nominal load electrical cycle.

To manage the unexpected $600 \mathrm{~W}$ pulse, the numerical optimization solver will be run every second in twenty second windows to optimally control the system. Therefore, the first part of the electrical load is not seen by the numerical optimization solver and at $t=21 \mathrm{~s}$, new information becomes available and the numerical optimization solver reacts. The fuel used by the numerical optimization method will be compared to the closed loop control system, which will run every second to manage the unforeseen load.

\subsubsection{Closed Loop Control}

Since the goal of the closed control is to respond to a $600 \mathrm{~W}$ step change in electrical load and ensure that the $S O C$ stays in the feasible range, a proportional controller based on $S O C_{\text {error }}$ was designed [87], which is shown in Figure 7.3.

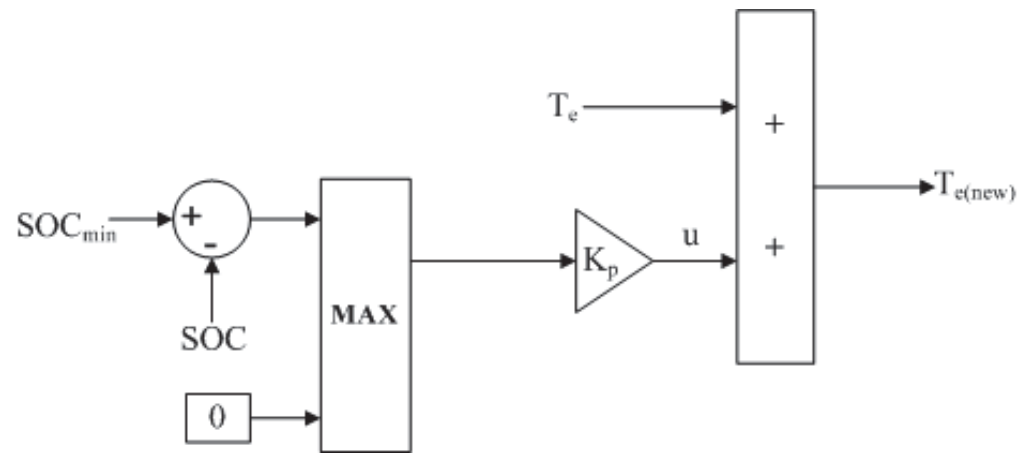

Figure 7.3: Closed loop control

Using this approach, the closed loop system presented in Equation (7.1) was employed. 


$$
T_{e_{\text {new }}}(t)=T_{e}(t)+u(t)
$$

where: $u(t)=k_{p} e(t)$

$$
\begin{aligned}
& e(t)=S O C_{\min }(t)-S O C(t), \text { if } e(t)<0 \text { then } e(t)=0 \\
& T_{e_{\text {new }}}(t) \leq T_{e_{\text {max }}}\left(\omega_{e}\right)
\end{aligned}
$$

It is a feedback controller based on the positive error between $S O C_{\min }$ and $S O C$. In other words, when $S O C$ is in the feasible range the error will be negative and therefore forced to zero and effectively turning off the control. Furthermore, $T_{e}$ still has to satisfy the constraints of the system and cannot be larger than $T_{e_{\max }}\left(w_{e}\right)$.

As with any closed loop controller, it is important to understand system behavior. One way to explore the response is to create a linearized state space representation and calculate the eigenvalues for the state matrix to assess stability. The linearized equations are shown in Equations 7.2, 7.3, and 7.4.

$$
\begin{gathered}
{\left[\frac{I_{v}^{\prime}(R+S)^{2}}{I_{e}^{\prime} K R}+\frac{I_{v}^{\prime} S^{2}}{I_{g}^{\prime} K R}+R\right] \delta \dot{\omega}_{m}=} \\
\delta T_{m}\left[\frac{(R+S)^{2}}{I_{e}^{\prime} R}+\frac{S^{2}}{I_{g}^{\prime} R}\right]+\delta T_{e}\left[\frac{(R+S)}{I_{e}^{\prime}}\right]+\delta T_{g}\left[\frac{S}{I_{g}^{\prime}}\right]- \\
\delta \omega_{m} \omega_{m_{0}}\left[\frac{C_{d} r_{t i r e}{ }^{3} a \rho}{K^{2}}\right]\left[\frac{S^{2}}{I_{g}^{\prime} K R}+\frac{(R+S)^{2}}{I_{e}^{\prime} K R}\right] \\
{\left[(R+S)+\frac{I_{e}^{\prime} S^{2}}{I_{g}^{\prime}(R+S)}+\frac{I_{e}^{\prime} K R^{2}}{I_{v}^{\prime}(R+S)}\right] \delta \dot{\omega}_{e}=} \\
\delta T_{m}\left[\frac{K R}{I_{v}^{\prime}}\right]+\delta T_{e}\left[\frac{S^{2}}{I_{g}^{\prime}(R+S)}+\frac{K R^{2}}{I_{v}^{\prime}(R+S)}\right]- \\
\delta T_{g}\left[\frac{S}{I_{g}^{\prime}}\right]-\delta \omega_{m} \omega_{m_{0}}\left[\frac{C_{d} r_{t i r e}{ }^{3} a \rho}{K^{2}}\right]\left[\frac{R}{I_{v}^{\prime}}\right]
\end{gathered}
$$




$$
S \dot{O} C(t)=\frac{-\delta T_{m} \eta_{m} \omega_{m_{0}}}{L}+\frac{\delta T_{g} \eta_{g} \omega_{g_{0}}}{L}-\frac{\delta \omega_{m} \eta_{m} T_{m_{0}}}{L}+\frac{\delta \omega_{g} \eta_{g} T_{g_{0}}}{L}
$$

Where:

$$
\begin{aligned}
L & =C_{\text {batt }} \sqrt{V_{o c}^{2}-4 R_{b a t t}\left(T_{m_{0}} \omega_{m_{0}} \eta_{m}-T_{g_{0}} \omega_{g_{0}} \eta_{g}+E_{p w r}\right)} \\
\delta \omega_{m}, \delta T_{m}, \text { etc. } & =\text { perturbed quantities } \\
\omega_{m_{0}}, T_{m_{0}}, \text { etc. } & =\text { nominal quantities }
\end{aligned}
$$

Combined with the control system detailed in Equations (7.1), the eigenvalues were calculated for the complete time series and plotted in Figure 7.4.

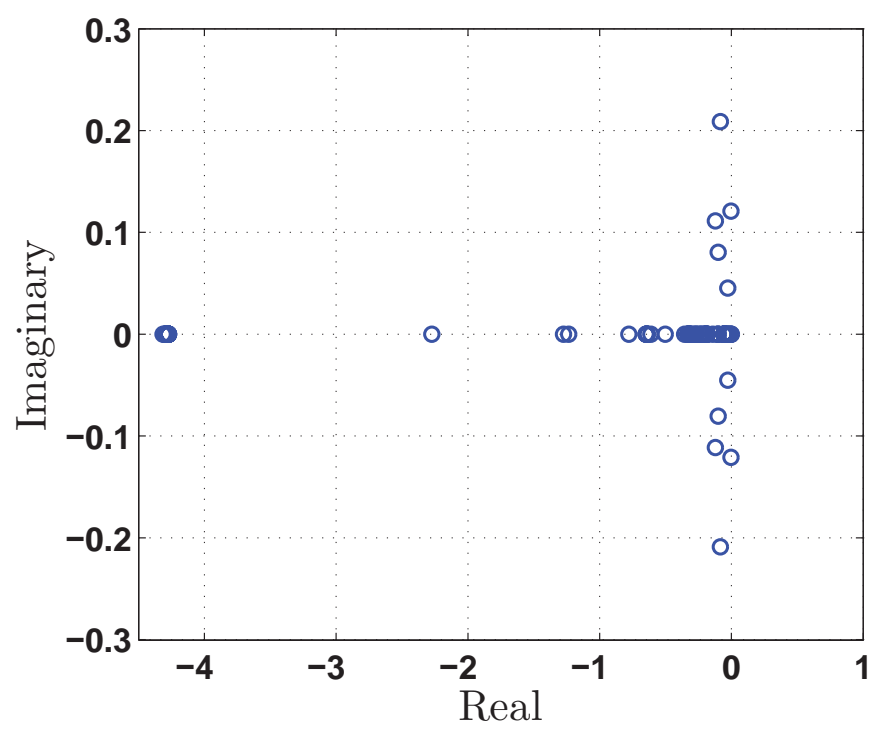

Figure 7.4: Closed loop eigenvalues

If any eigenvalues had been in the right half plane, the closed loop system would be unstable. Since the system is time-varying, it's not sufficient to have all the eigenvalues in the left half plane to ensure stability. The mupad code can be found in Appendix C. 


\subsubsection{Controller Comparison Results}

Figures 7.5 and 7.6 show the torque trajectories for the engine and generator, respectively, for each of the different control systems. The cost for the optimizer was .1213 liters and the cost for the closed loop control was .1812 liters.

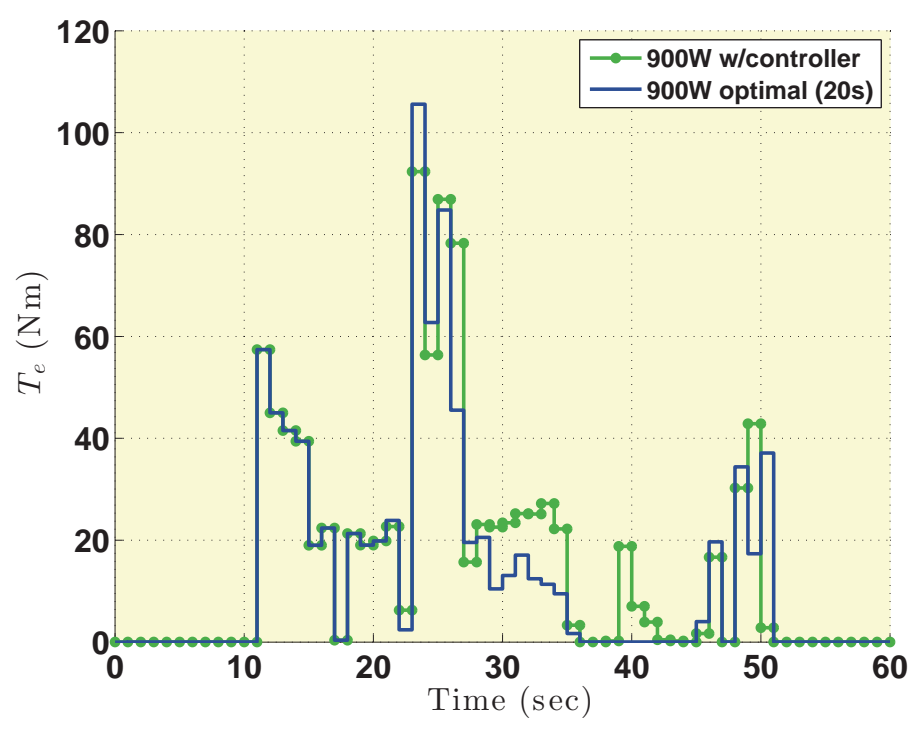

Figure 7.5: Engine torque trajectories

The major difference in the two results can be seen around the thirty second point in Figure 7.5, engine torque trajectories, when the closed loop controller is reacting to the added electrical load. The controller only has one option when the SOC gets close to its minimum value which is to increase engine torque and consume more fuel. This is further explained in Figure 7.7, which is a plot of the instantaneous fuel used for both control systems. Again, at $t=29$ seconds the closed loop controller forces a large fuel consumption event to manage the added electrical load.

Optimal torque trajectories create the optimal $S O C$, found in Figure 7.8, which shows how the closed-loop controller overshoots and charges the battery more than necessary and keeps it from meeting the final $S O C$ value. Applying an integral term to the closed loop controller would not help in this particular situation, because the 


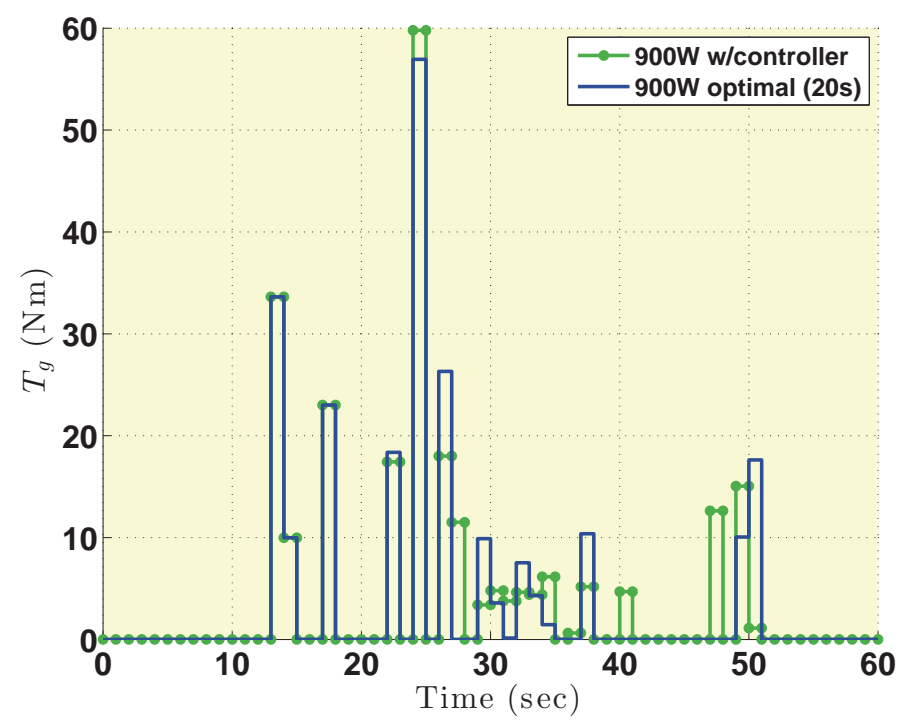

Figure 7.6: Generator torque trajectories

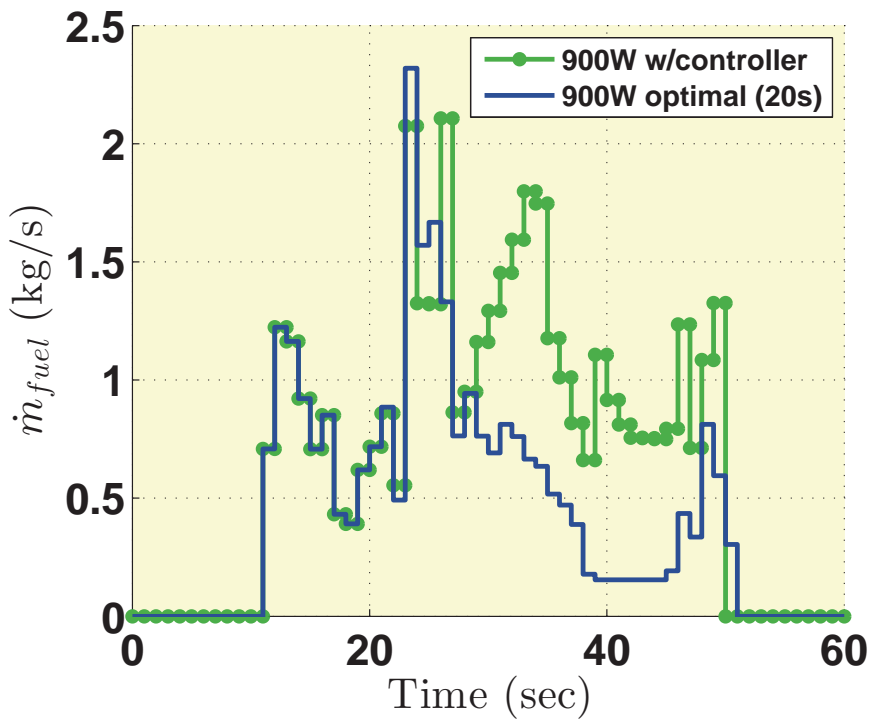

Figure 7.7: Instantaneous fuel used

$S O C$ needs be lower.

It is important to note that the propulsion duty cycle was fulfilled as shown by Figure 7.9 , which is a plot of the motor speed for both controls methods. Recall 


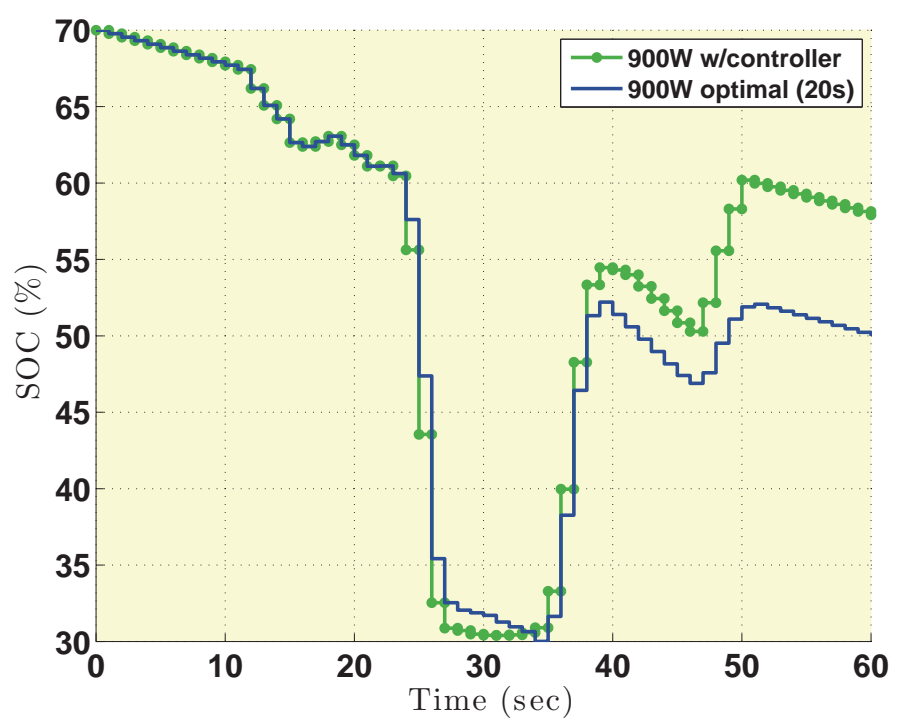

Figure 7.8: Battery state of charge

that motor speed is directly related to vehicle speed through the tire radius and the final drive ratio. Furthermore, the constraints of the system were met by each of the control methods as shown in Figure 7.10, which is a plot of constraint number versus scaled constraint violation. As explained in Chapter 6, all of the constraints were formulated as equality constraints and should be equal to zero. The small values are effectively zero and taken as numerical errors only. The plots for the remaining parameters can be found in Appendix D for reference.

\subsubsection{Stationary Grid Requirement}

While this analysis provides some interesting information about the vehicle performance, it is desirable to compare the methods with an equal $S O C_{\text {final }}$ value. Having the same $S O C$ at the conclusion of the maneuver, creates a level comparison of the two systems from an energy view point. Since the closed-loop proportional controller is not capable of controlling to an $S O C_{\text {final }}$ value, the optimization was re-run with $S O C_{\text {final }}=58 \%$, which was where the closed-loop control system concluded. 


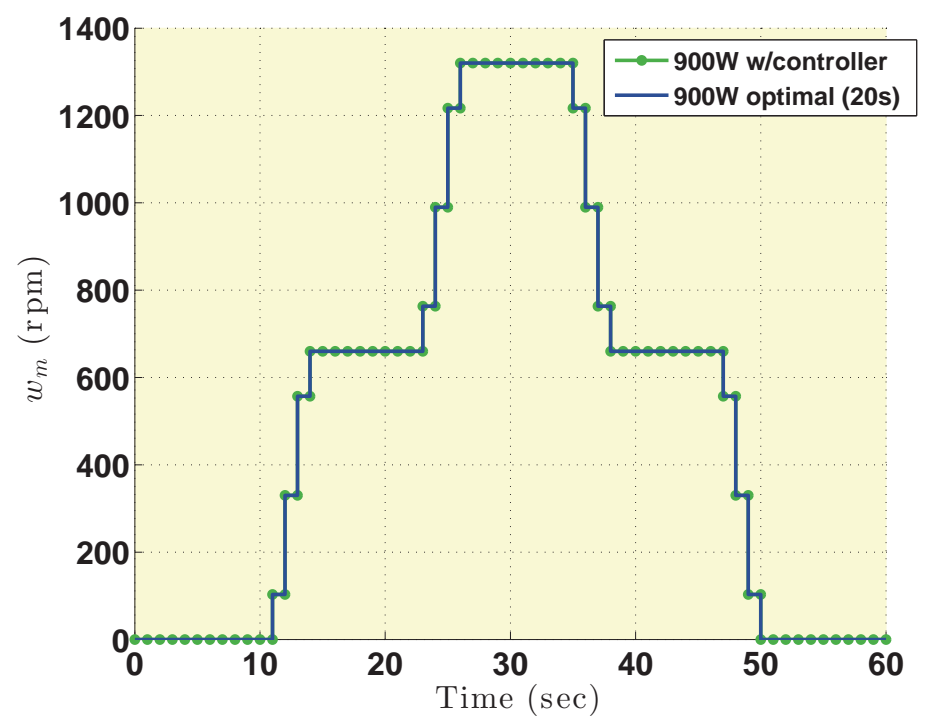

Figure 7.9: Motor speed

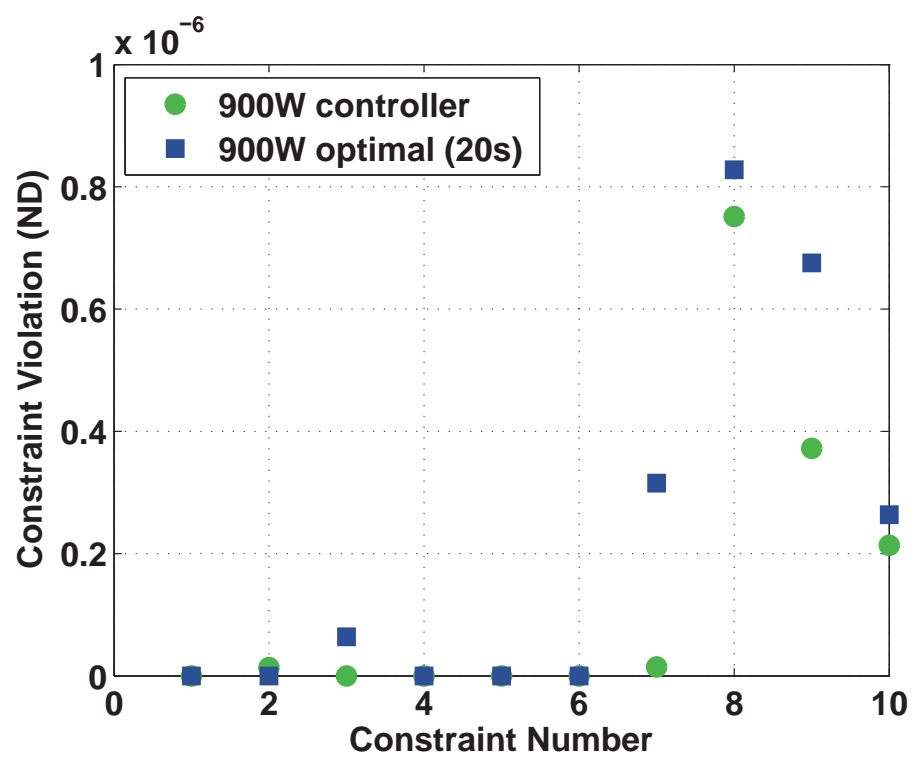

Figure 7.10: Constraints for both control systems

Figure 7.11 shows the cost in liters for the following cases:

1. case 1 - closed loop controller with $S O C_{\text {final }}=58 \%$

2. case 2 - numerical optimization with $S O C_{\text {final }}=50 \%$ 
3. case 3 - numerical optimization with $S O C_{\text {final }}=58 \%$

Case 2 uses the least amount of fuel as expected. However, case 3 with the higher $S O C_{\text {final }}$ still uses less fuel than closed loop control.

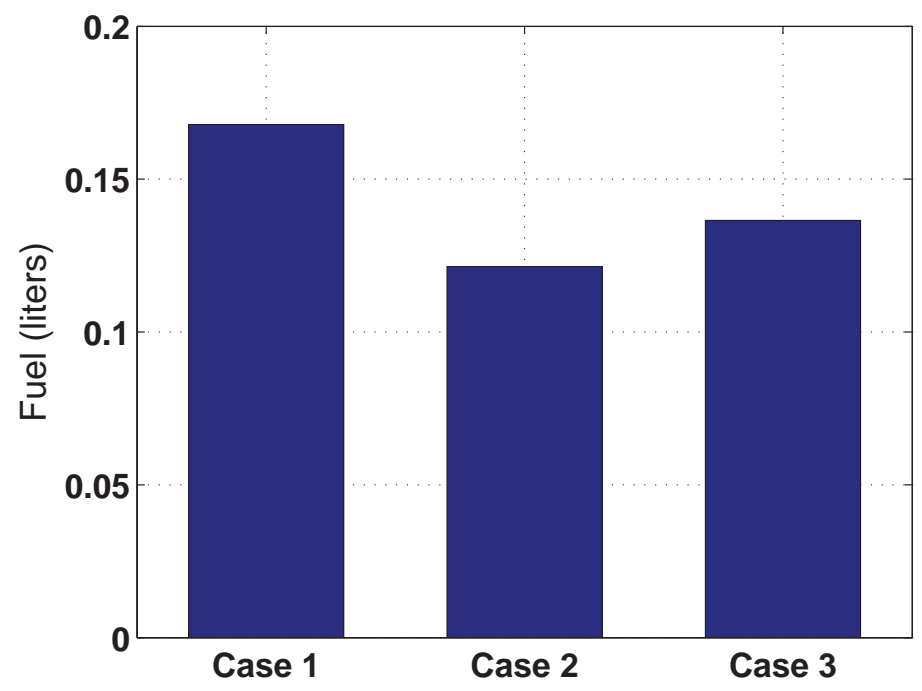

Figure 7.11: Cost of each case

Figures 7.12, 7.13 illustrate the engine torque and generator torque for each case, respectively. Case three has increased engine torque and generator torque through out the cycle, but what is most interesting is the $S O C$ plot, shown in Figure 7.14.

The optimal torque trajectories create an optimal SOC trajectory, which is demonstrated in Figure 7.14. For case three, the $S O C$ increases early in the cycle at approximately $t=14 s$ to prepare for the increased $S O C_{\text {final }}$ requirement at the end of the cycle.

In addition, the instantaneous fuel used, shown in Figure 7.15, explains how the optimizer used more fuel at the beginning to prepare for the added energy requirement at the end of the cycle, which proved to be more efficient than the closed loop control.

It is essential to note that the vehicle was meeting its mobility drive cycle as shown 


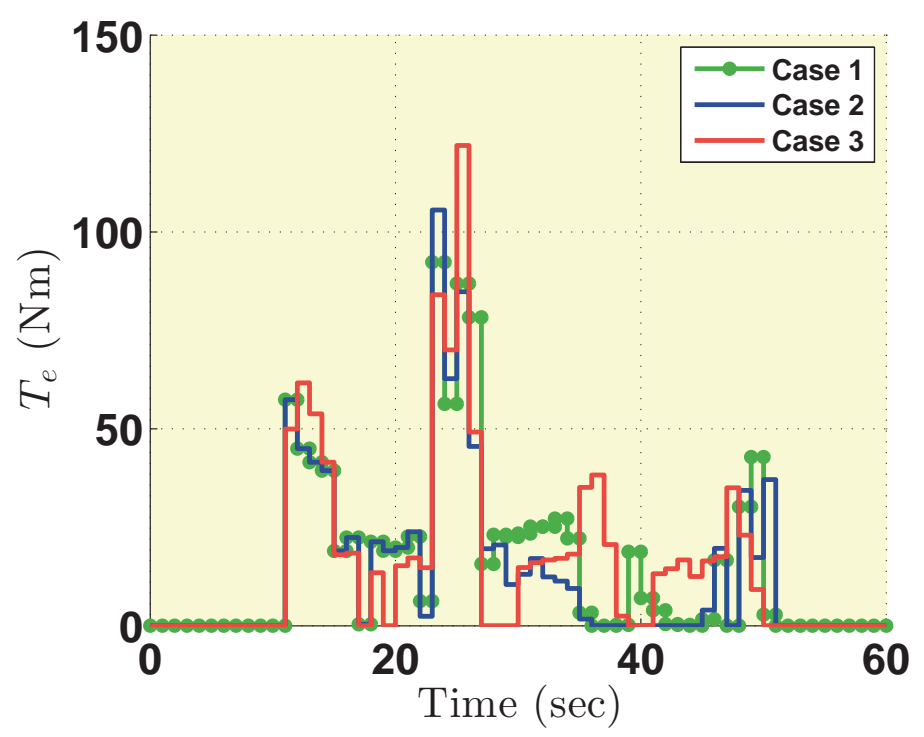

Figure 7.12: Engine torque trajectories with new case

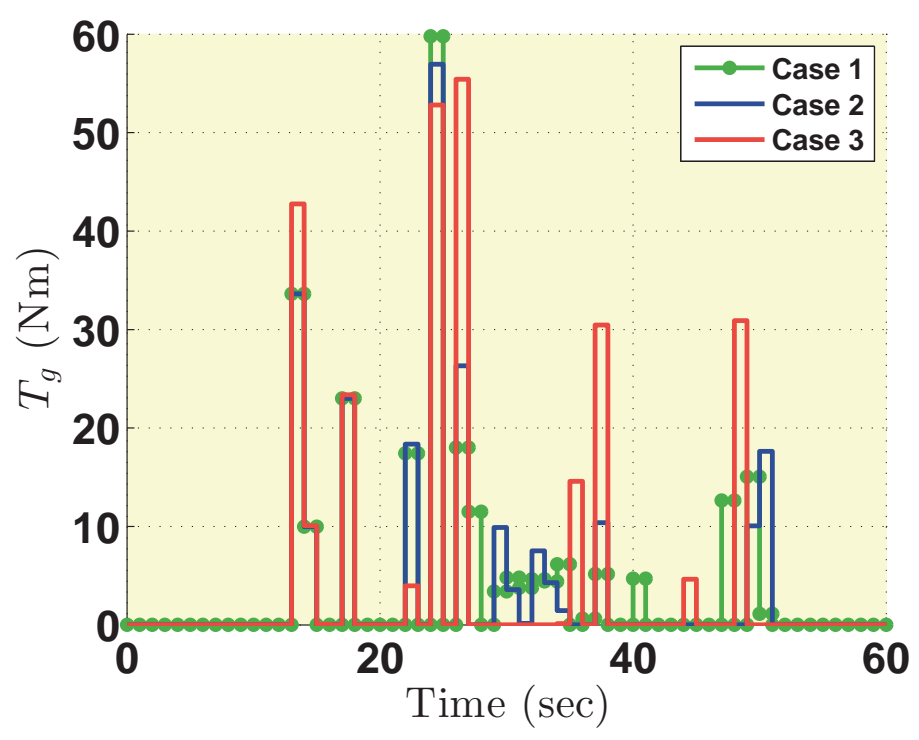

Figure 7.13: Generator torque trajectories with new case

in Figure 7.16, which is a plot of motor speed, $\omega_{m}$, versus time for each of the cases. In addition, the constraints of the optimization were met as shown in Figure 7.17, which is a plot of the scaled constraints, which were scaled by their maximum value. The small values are considered numerical errors only. The plots for the remaining 


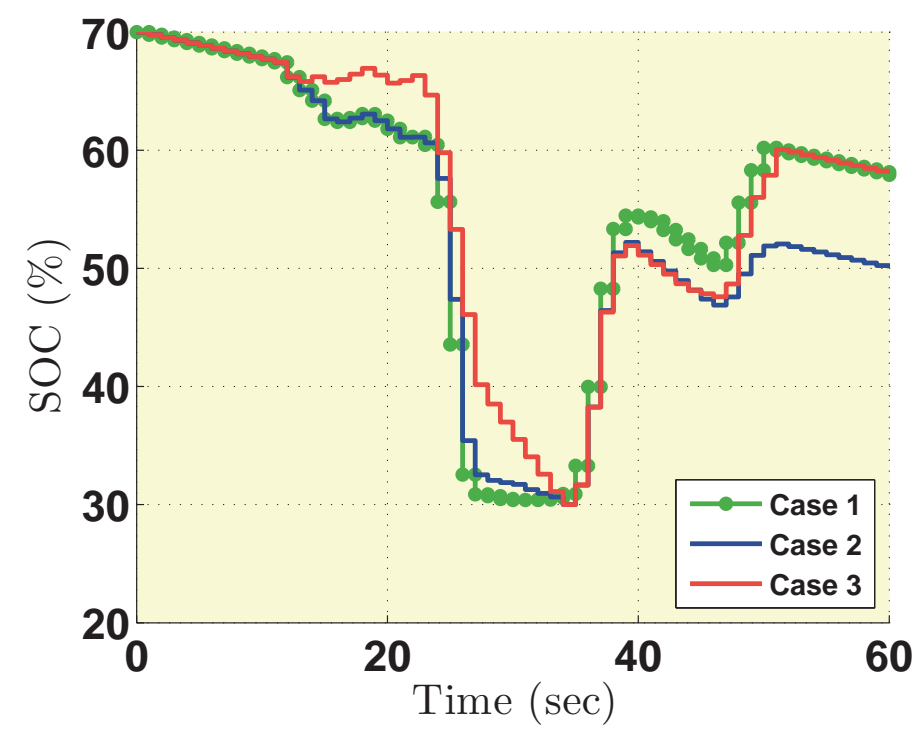

Figure 7.14: Battery state of charge trajectories with new case

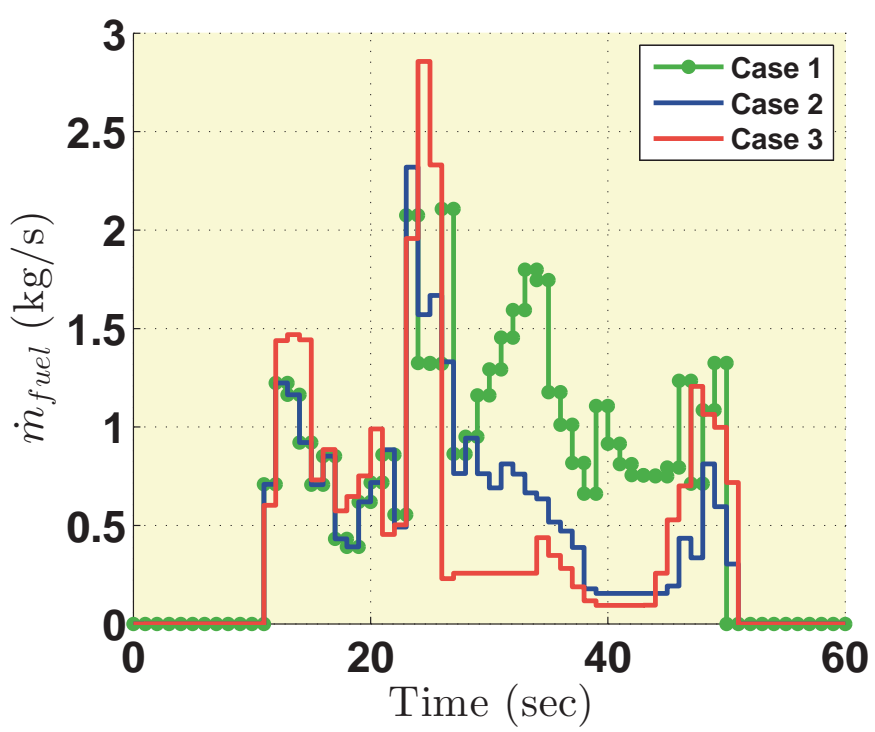

Figure 7.15: Instantaneous fuel used for each case

parameters can be found in Appendix D for reference. 


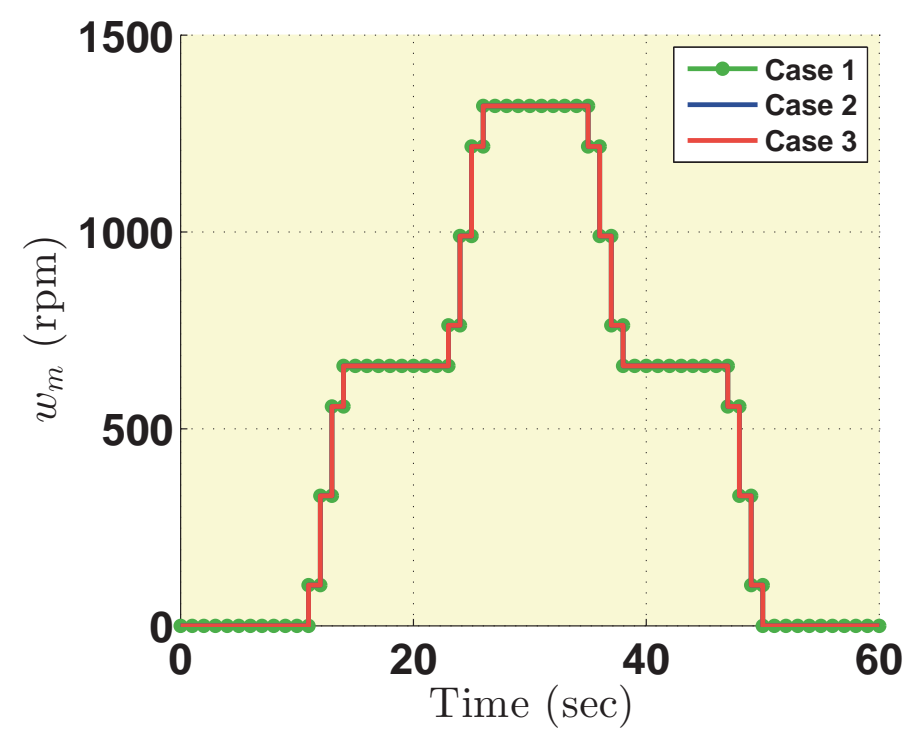

Figure 7.16: Motor speed for each case

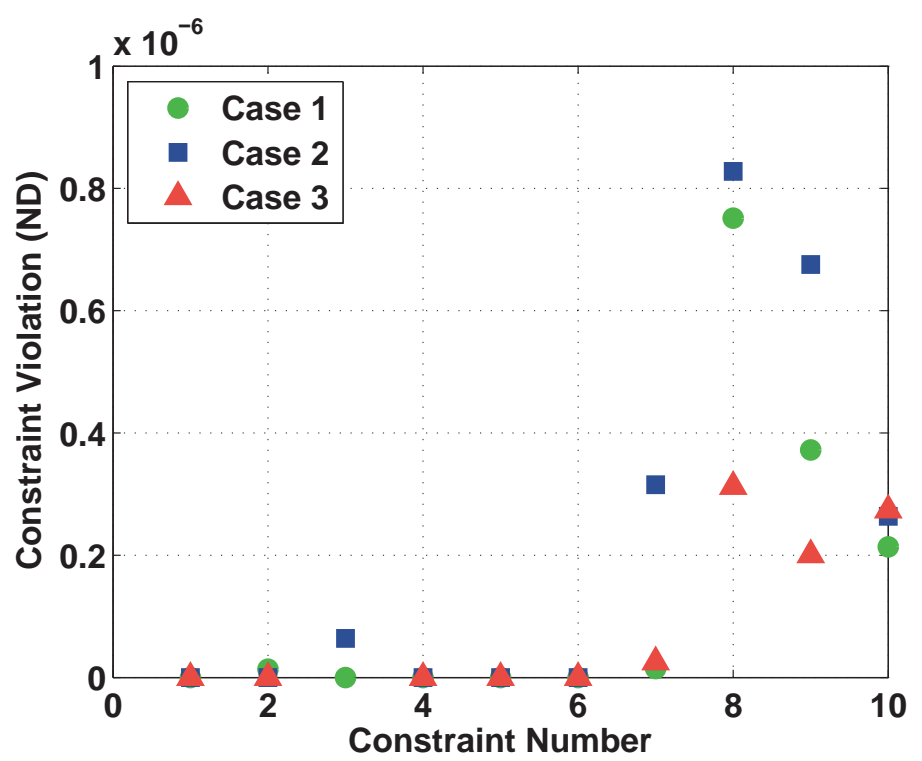

Figure 7.17: Constraints for each case 


\subsubsection{Electrical Cycle Parametric Study}

It is important to understand how location of the $600 \mathrm{~W}$ electrical step change effects the optimal solution. More specifically, is the optimal solution still more efficient than the closed loop controller when the location of the step change is varied. Therefore, a parametric study was performed that varied the start of the electrical cycle step change from $t=1 \mathrm{~s}$ to $t=19 \mathrm{~s}$, shown in Figure 7.18. Recall that the original electrical cycle step change started at $t=16 \mathrm{~s}$ and the numerical optimization solver ran at $t=20 \mathrm{~s}$, also shown in Figure 7.18.

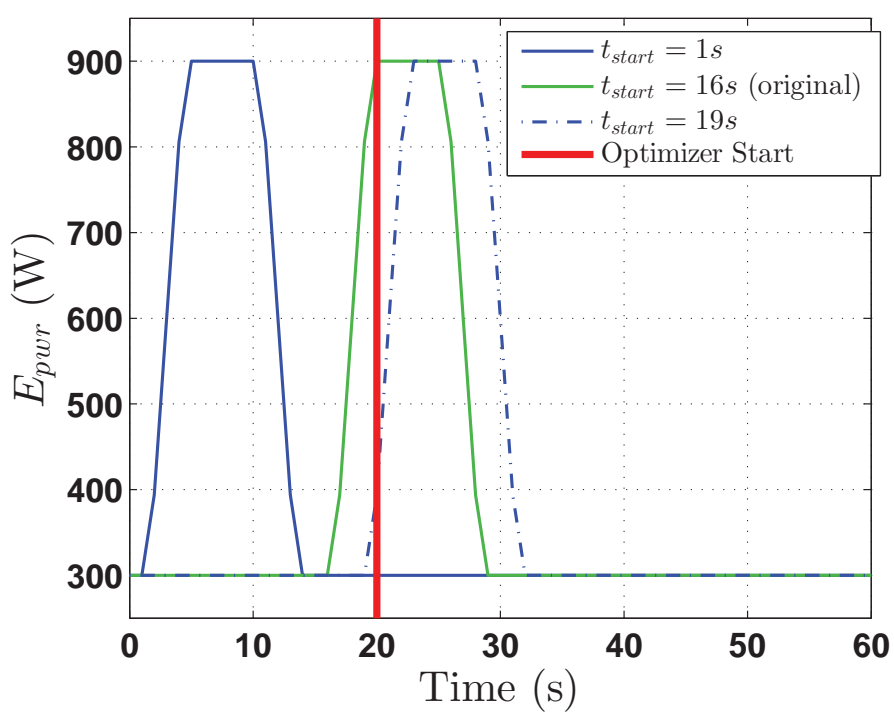

Figure 7.18: Electrical duty cycle test definition

For this work, the numerical optimization solver still re-optimizes at $t=20 \mathrm{~s}$. In addition, the propulsion cycle, shown in Figure 7.1 and $S O C_{\text {final }}=50 \%$ were used. The closed loop control operates as previously explained. The fuel consumed for both systems were compared and the results are shown in Figure 7.19. For each of the different start times, the numerical optimization solver is more efficient than the closed loop control. Furthermore, Figure 7.19 shows that the optimal solution is invariant with respect to the location of the electrical duty cycle step change. Lastly, the vehicle was fulfilling the mobility cycle requirement and the constraints were met. 


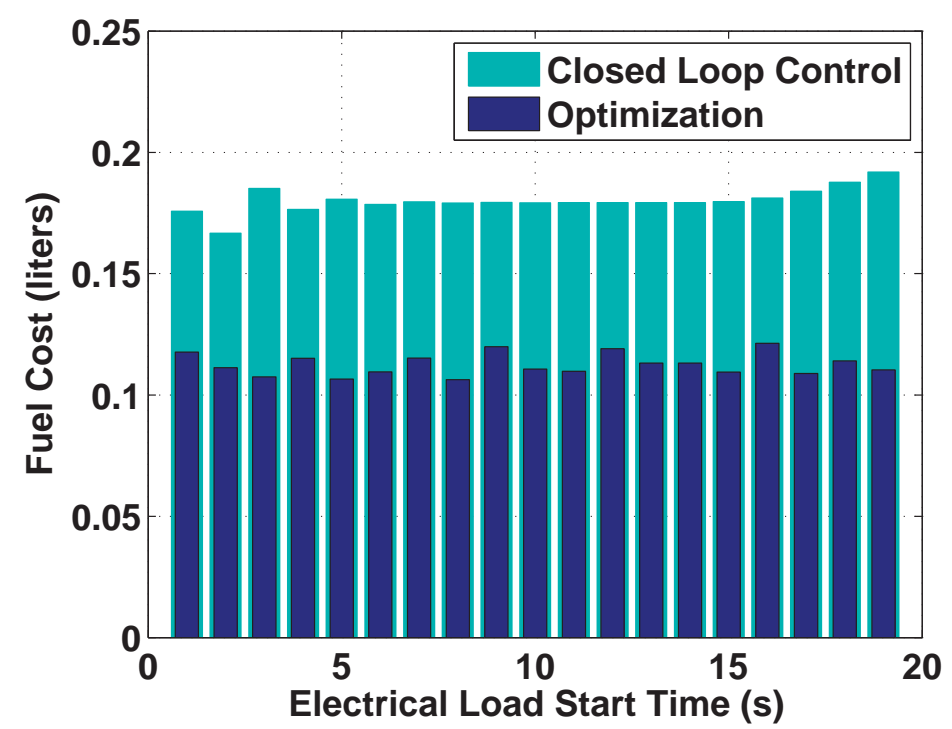

Figure 7.19: Cost of comparison for electrical duty cycle sensitivity analysis

\subsection{Design Optimization}

As explained in Chapter 3, the U.S. Army typically treats all an electrical requirement as a constant load, however this is typically not the operational reality. This section describes how the framework can be expanded to show the effect of electrical duty cycle on not only fuel use, but also battery capacity sizing. 


\subsubsection{Problem statement}

Recall Figure 7.2, which shows three different electrical duty cycles:

1. an actual case of a $300 W$ constant load with a $600 W$ step change

2. a nominal case of $300 W$ constant load

3. a worst case of a $900 W$ constant load

These different electrical cycles will not only effect the fuel used, but also the battery sizing. Therefore, the optimization problem can be re-written in the following manner, find $T_{e}, T_{g}, T_{f b}$, and $C_{b a t t}$ such that:

$$
\begin{array}{cl}
\text { minimize: } & J=w_{1} \sum_{t=t_{0}}^{t_{f}} \dot{m}_{f u e l}\left(T_{e}, T_{g}, T_{f b}\right)+w_{2}\left(C_{b a t t}\right) \\
\text { subject to: } & 0 \leq T_{e} \leq T_{e_{\max }}\left(\omega_{e}\right) \\
& \leq T_{g} \leq T_{g_{\max }}\left(\omega_{g}\right) \\
& T_{m_{\min }}\left(\omega_{m}\right) \leq T_{m} \leq T_{m_{\max }}\left(\omega_{m}\right) \\
& 0 \leq T_{f b} \leq T_{f b_{\max }} \\
& S O C_{\min } \leq S O C \leq S O C_{\max } \\
& C_{b a t t_{\min }} \leq C_{\text {batt }} \leq C_{\text {batt }} \max \\
\text { where: } & w_{1}, w_{2} \text { are weighting factors. }
\end{array}
$$

To ensure a fair comparison, $S O C_{\text {init }}$ and $S O C_{\text {final }}$ have to be adjusted so that the total energy in the system is equal as the battery is resized. This is first addressed by combining the energy equation and Ohm's law shown in Equations 7.8, 7.9, and 7.10, 
to determine that energy, $\mathbf{E}$ (joules), is equal to capacity, $A-s$, multiplied by the voltage, $V$.

$$
\begin{aligned}
\mathbf{E} & =P * t \\
P & =i * V \\
\therefore \mathbf{E} & =(i * V) t=(i * t) V=C * V
\end{aligned}
$$

The actual battery capacity, $C_{b a t t_{a c t}}$, is defined by the actual $S O C$, shown in Equation 7.11 .

$$
C_{b a t t_{a c t}}=S O C_{a c t u a l} * C_{b a t t}
$$

Combining Equations 7.10 and 7.11,

$$
\mathbf{E}=S O C_{a c t} * C_{b a t t} * V * 3600 \frac{s e c}{h r}
$$

Finally, by setting the energy, E, to a constant value at initialization. The initial $S O C$ can be determine by Equation 7.13.

$$
S O C_{\text {init }}=\frac{\mathbf{E}}{C_{\text {batt }} * V * 3600 \frac{\mathrm{sec}}{\mathrm{hr}}}
$$

Every time the battery capacity is changed by the optimizer a new $S O C_{\text {init }}$ is determined based on the constant energy in the system. In addition, $S O C_{\text {final }}$ was scaled in the same manner, again, to ensure that the total amount of energy in the system is constant, defined by the original $C_{b a t t}=6.5 A-h r$ and $S O C=30 \%$. The same propulsion duty cycle, found in Figure 7.1, was used. The system was optimized every second over the full sixty seconds. 


\subsubsection{System Component Design Results}

The results for the cost of each case is found in Figure 7.20, which is a plot of the cost of fuel in liters and battery capacity in ampere-hours.

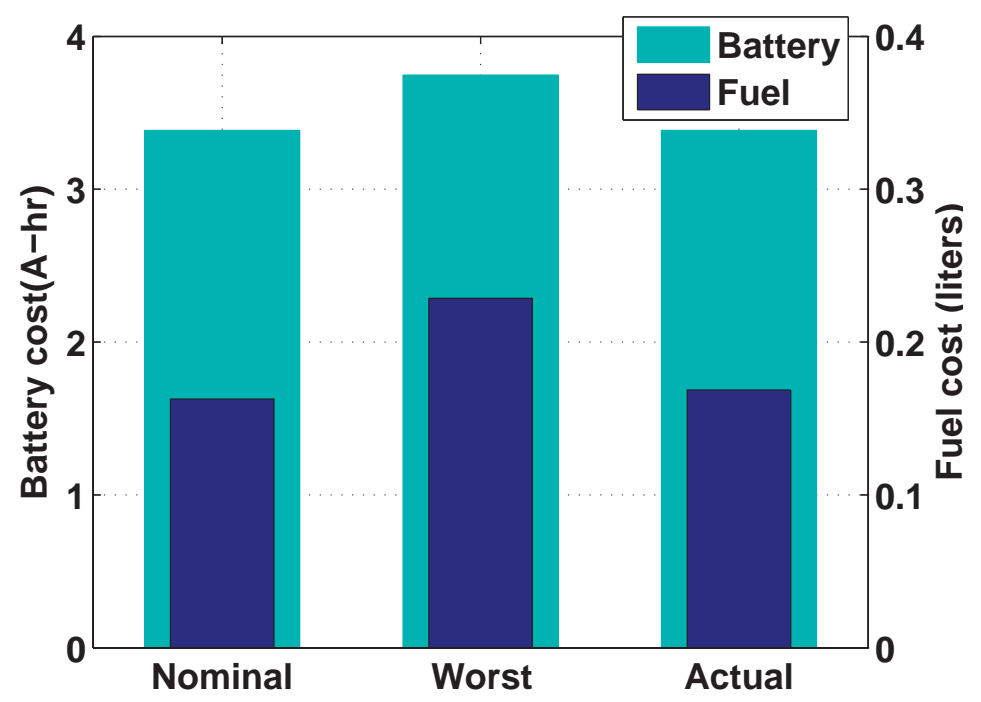

Figure 7.20: Battery and fuel cost for different electrical duty cycles

This plot is particularly interesting because it not only shows the fuel economy impacts related to the different electrical loads, but it also shows a design impact. When the worst case cycle is used to design a system, there is a risk of over design - the suggested battery is $10.70 \%$ larger than needed. The converse is also true, which makes defining the cycle valuable in the hardware design process. Furthermore, this optimization tool created for this work is increasingly valuable in all facets of military hybrid vehicle research.

For further examination, Figures 7.21, 7.22, and 7.23, show the optimal torque trajectories for the engine, generator and brake force.

It is apparent from these plots that the engine and generator need to provide more torque to fulfill the worst case electrical duty cycle. This is particularly evident 


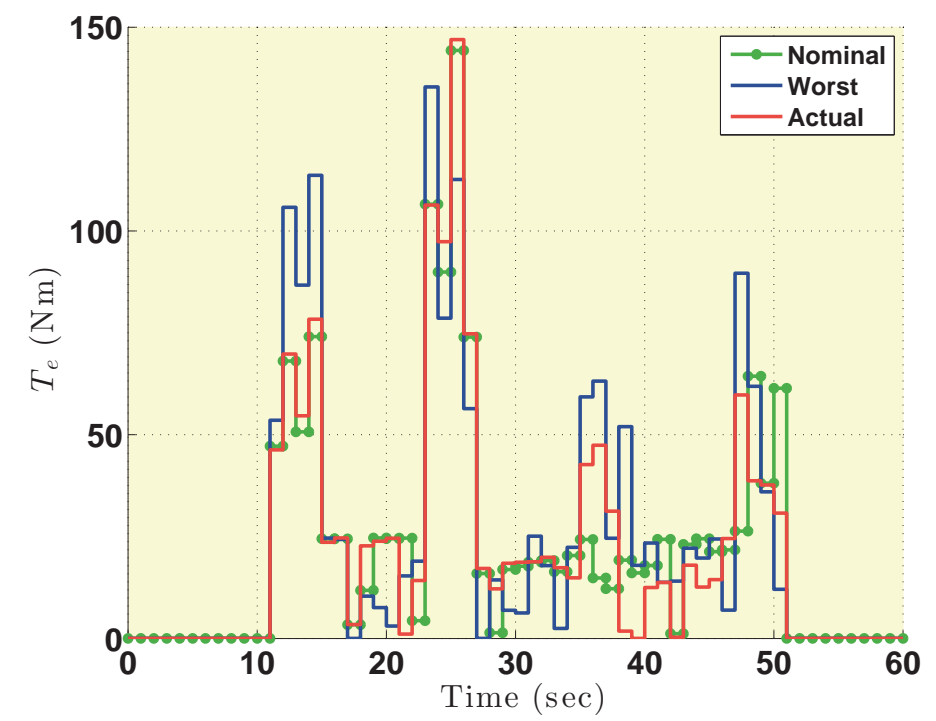

Figure 7.21: Engine torque trajectories for each electrical duty cycle

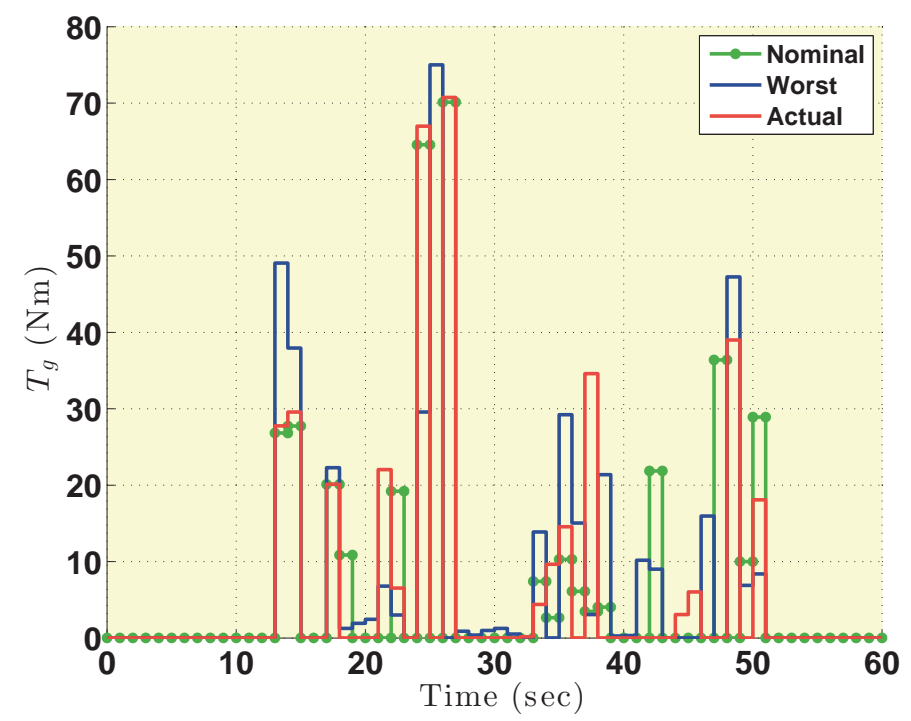

Figure 7.22: Generator torque trajectories for each electrical duty cycle

between ten and twenty seconds and thirty and forty seconds on the engine torque plot (Figure 7.21). To further understand the differences, Figures 7.24 shows the engine speed, $\omega_{e}$ trajectories for each case. These trajectories also explains the added fuel cost for the worst case electrical duty cycle. The engine speed is much faster for 


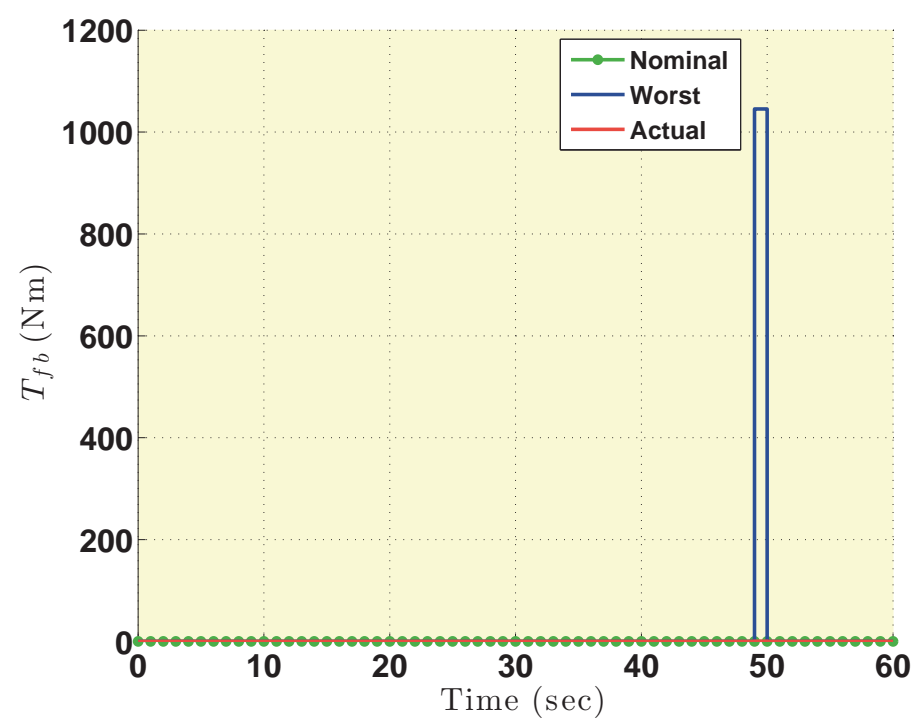

Figure 7.23: Brake force torque trajectories for each electrical duty cycle

this case.

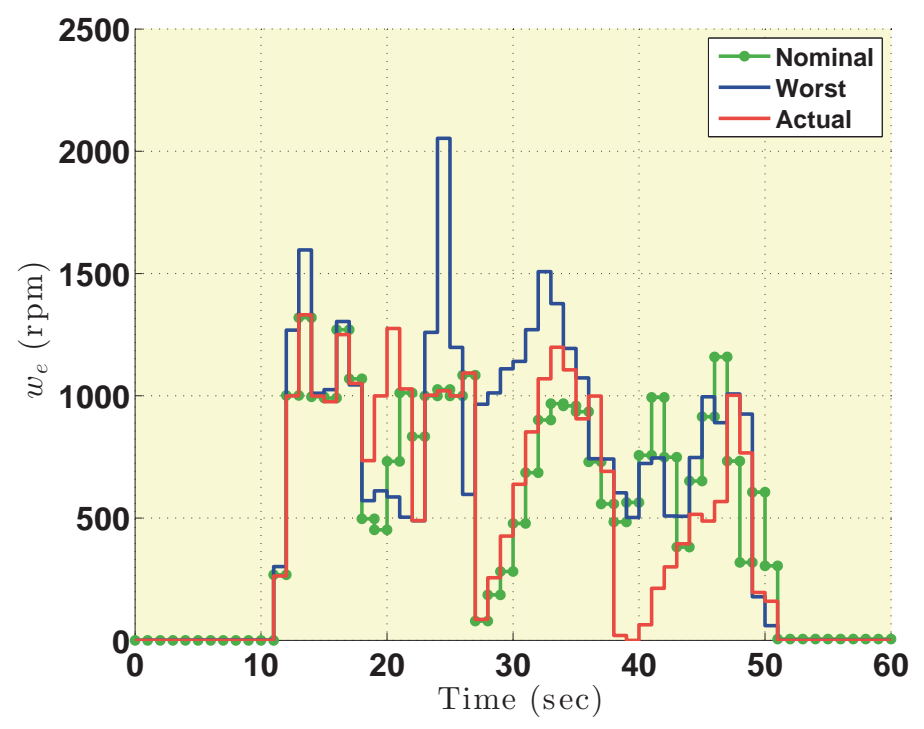

Figure 7.24: Engine speed for each electrical duty cycle

The optimal torque trajectories create an optimal $S O C$ trajectory, which is shown in Figure 7.25. The $S O C$ for the nominal and actual case are quite similar, with some small differences at $t=22 \mathrm{~s}$ and $t=35-45 \mathrm{~s}$. These differences make sense 
as the system is accounting for the added $600 \mathrm{~W}$ load for a short period of time. It also makes sense that the major differences are when the vehicle is decelerating and the optimal solution is taking advantage of the added energy in the system for regenerative braking. The worst case shows the most difference and this is due to the larger battery, which results in a lower $S O C_{\text {init }}$ and $S O C_{\text {final }}$. The larger battery is a somewhat of a disadvantage, because at $t=35$ seconds, when all three of trajectories hit $S O C_{\text {min }}$, the large battery has more energy that it has to bleed off to reach the correct $S O C_{\text {final }}$, which is shown in Figure 7.23 , the brake force torque trajectory at $t=45 s$.

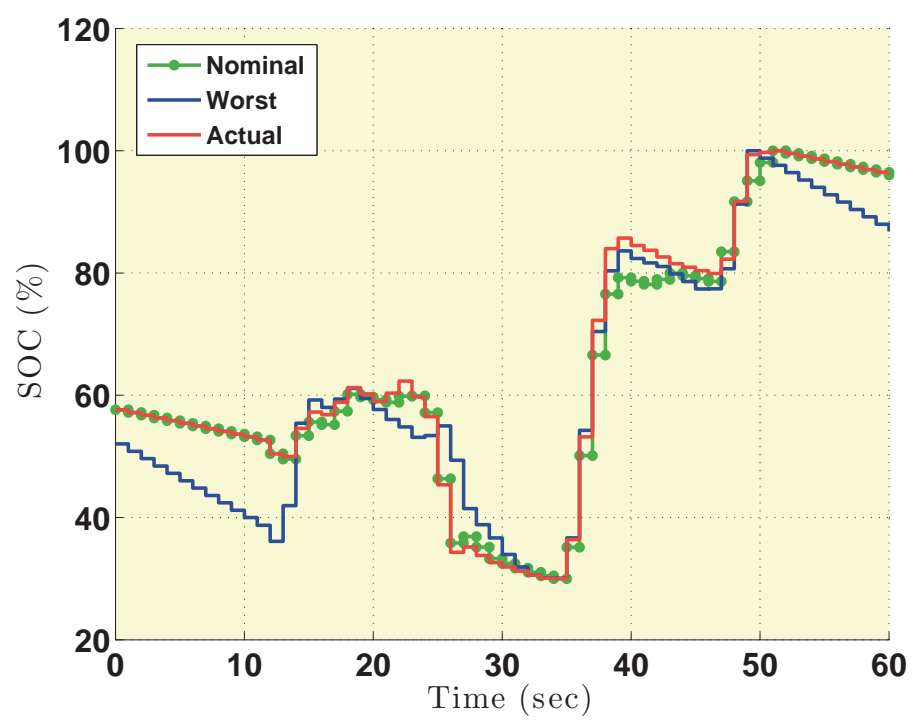

Figure 7.25: Battery state of charge for each electrical duty cycle

Next, the fuel used, shown in Figure 7.26 further depicts what has already been shown in the preceding figures. The most fuel was used by the worst case, even with the larger battery. The fuel use would be even greater with the same battery size as in the nominal and actual case. The actual case uses more fuel than the nominal case, which is as expected.

Finally, the propulsion cycle and constraints were met as shown by Figures 7.27 and 7.28, respectively. As before, the small values for the scaled constraints are considered numerical errors. The plot for the remaining parameters can be found in Appendix E for reference. 


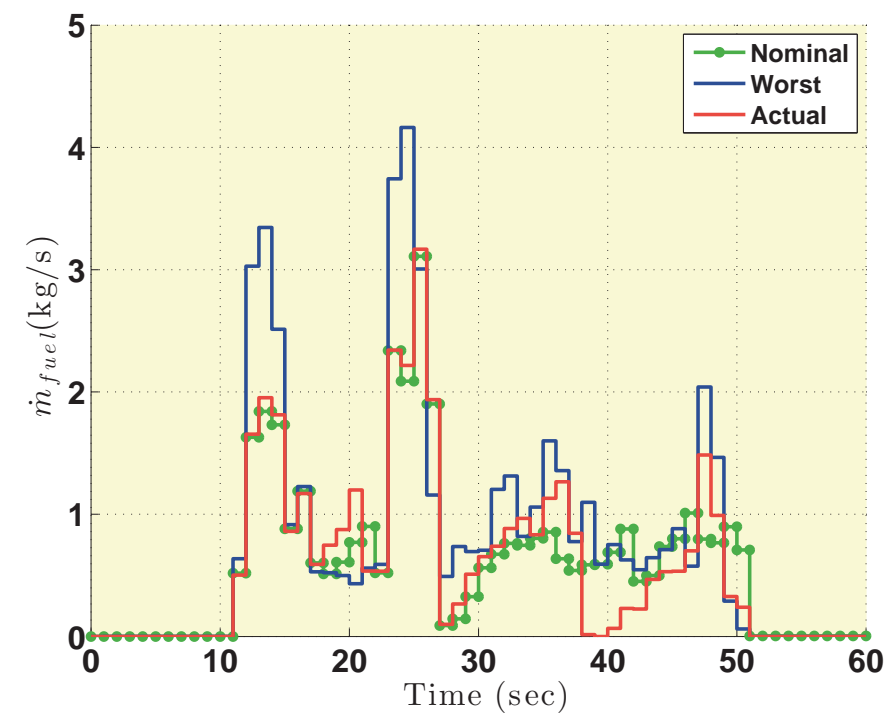

Figure 7.26: Fuel used for each electrical duty cycle

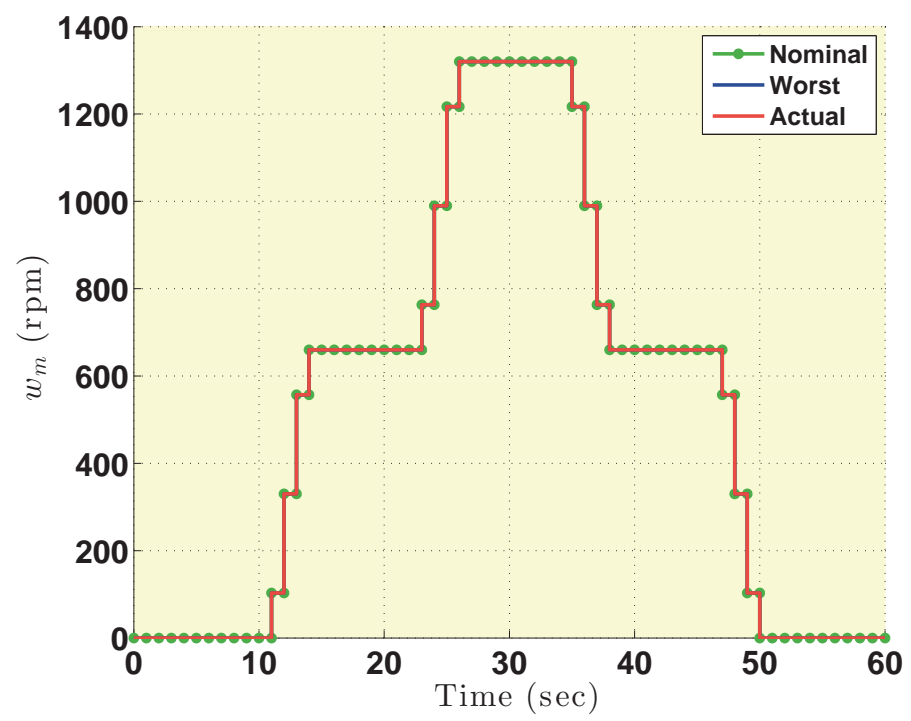

Figure 7.27: Motor speed for each electrical duty cycle 


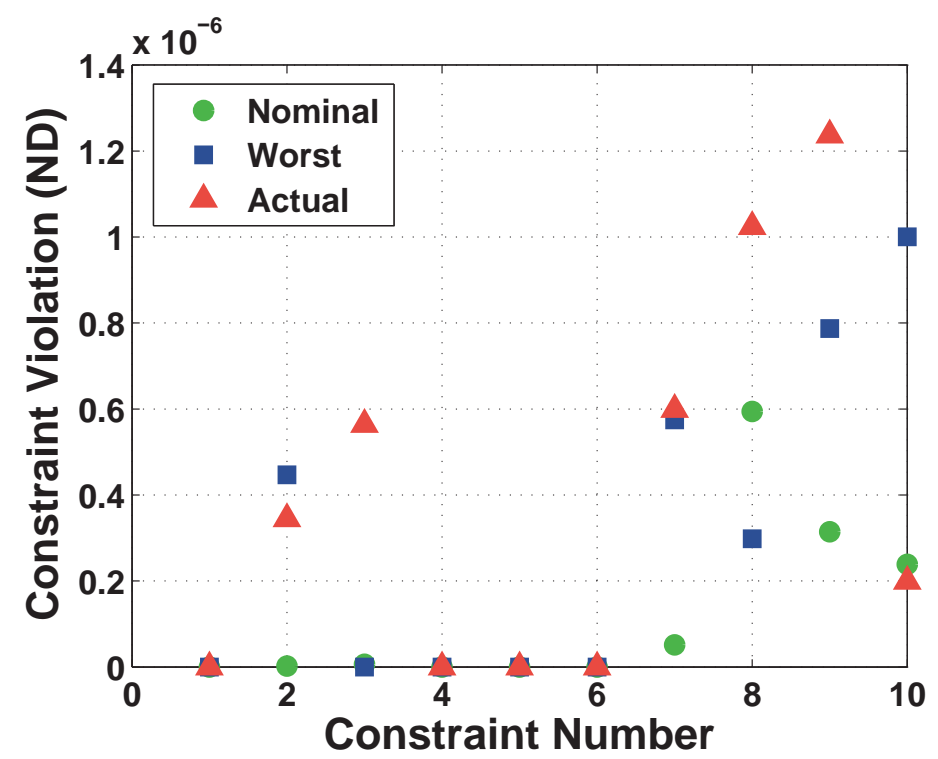

Figure 7.28: Constraints for each electrical duty cycle

\subsection{Summary}

The results shown in this chapter prove that the numerical optimization method can decrease fuel consumption when compared with proportional controller. Additionally, this method can be expanded to include vehicle design parameters, which produced results that explained the effect of the electrical duty cycle on the battery sizing. Ultimately, the results have broader impact in the sense that they lead toward model predictive control, which may produce a significant fuel economy improvement in military hybrid vehicles over traditional control systems. 



\section{Chapter 8}

\section{Summary and Conclusions}

\subsection{Summary}

Although a large amount of work has been done to show that military hybrid vehicles could improve fuel economy, a military hybrid vehicle has yet to be fielded. The benefits of a military hybrid vehicle are difficult to translate into a tangible mission energy reduction. This is due to the lack of applicable duty cycles and the use of a holistic view of energy consumption by the military community. A method to determine the benefit of a military hybrid vehicle had yet to be quantified, especially related to microgrids and $\mathrm{V} 2 \mathrm{G}$ capability.

This dissertation presented a method to quantify the benefits of a military hybrid vehicle by regarding a military hybrid vehicle as a microgrid. This novel concept allowed for the creation of generic and expandable multiple input numerical optimization method that can be used for optimal control and vehicle design. The first 
area of interest for this method was developing a duty cycle that represented a relevant mission scenario, including a propulsion duty cycle, electrical duty cycle and stationary grid requirements.

The propulsion cycle contained the required maneuvers for the mission profile, including variations in speed and steady state operation. The electrical cycle consisted of three different duty cycles, nominal, actual and worst case, to show the effect on fuel used and system design. Finally, the concept of state of charge optimization with respect to vehicle microgrids and stationary microgrids was introduced by the final battery state of charge, $S O C_{\text {final }}$, requirement.

An applicable vehicle model was created to demonstrate the concept. The Toyota ${ }^{\circledR}$ Prius hybrid vehicle was chosen because it is detailed enough to show the effects of the duty cycles, but does not have any publication restrictions. It contains a common hybrid vehicle architecture of an internal combustion engine and two electrical machines in a series-parallel hybrid configuration. Furthermore, there is an abundance of information regarding the vehicle performance and model parameters, which can provide cursory validation.

Due to this method's complexity, early research focused on only a propulsion duty cycle, a single input $(S \dot{O} C)$ optimization and a simplified vehicle model. The goal of this work was to understand if fuel could be minimized by using the concept of a vehicle microgrid. This effort demonstrated that using a two-step optimization method can reduce fuel used over a given duty cycle. Additionally, there were a number of lessons learned regarding initial guess determination, cost function construction and charge and discharge frequency of batteries. However, this early was limited in use and expandability.

Building on this early success, a multiple input numerical optimization method was created that could take into account multiple duty cycles as well as vehicle parameters. The creation of this method also included the development of constraints and a reliable numerical integration methodology. Additionally, it can be used to trade fuel usage versus vehicle component parameters, such as battery storage capacity. 
The final implementation of this method consisted of two applications, namely, real time control and system design optimization. The application of real time control for a typical military scenario included a prescribed vehicle mission with a known propulsion cycle, nominal electrical load and final grid requirement. An large unknown step change in electrical load was then introduced in addition to the nominal load and the optimizer reaction and control was compared to a traditional closed loop control system. It was shown that the new control method used less fuel over the scenario, while fulfilling the propulsion, electrical, $S O C_{\text {final }}$, and constraint requirements, when compared to the closed loop control method.

The next application expanded the optimization problem to include not only fuel, but the battery capacity as well. The three different electrical duty cycles - nominal, actual and worst case - were used along with the propulsion duty cycle and $S O C_{\text {final }}$ requirement. When the worst case electrical cycle was utilized, the optimal solution included an increased battery size, which illustrates the importance of the electrical duty cycle and holistic system analysis.

Reducing fuel use on the battlefield not only reduces cost, but saves lives. Decreasing the number of fuel convoys, reduces the amount of time that soldiers are in harms way. To realized this actuality, realistic and trustworthy vehicle requirements are needed to drive full capability vehicle design, which enables solder survivability and mission success. This numerical optimization methodology not only supports both fuel reduction and system design individually, it allows for understanding of the trade off between them since they are intrinsically tied together in a complex way. 


\subsection{Conclusions}

The main conclusions, based on the results of the previous chapters, are listed below.

- By assembling the results of an exhaustive literature search on military hybrid vehicles, this dissertation was able to identified the effect of duty cycles on military hybrid vehicle performance. The literature search also highlighted the lack of consistent requirements when evaluating military hybrid vehicles. Furthermore, it was also concluded that the electric duty cycle, which is important to the military mission, was deemphasized or neglected.

- The basis function $S O C$ optimization served as a proof of concept and demonstrated that fuel consumption could be reduced in a military hybrid vehicle using this optimization method. While this method is not appropriate for vehicle control due to the two-step methodology, it did prove to be an extremely powerful tool for the vehicle design and requirements development.

- This dissertation concluded that neglecting any applicable duty cycle, such as the electric duty cycle, will result in lower vehicle efficiency and potentially poorly sized components. In the example presented, the electrical duty cycle was included in optimizing the MHVM and was proven to impact the battery design. Furthermore, this example demonstrated that a MHVM battery would be $10.7 \%$ larger than optimal as a result of design based on worst case electrical duty cycle.

- The numerical optimization method produced a fuel economy improvement of $18.7 \%$ when compared to a traditional proportional controller. This conclusion has the potential to be paradigm shifting as the results indicate that utilizing model predictive control could drastically improve overall energy efficiency over more traditional control methods. 


\subsection{Contributions}

Overall, this work provided a greater understanding of military hybrid vehicles with respect to operational energy by:

- Regarding a military hybrid vehicle as a microgrid, which allows for generic and expandable optimization

- Creating a method for a holistic view of duty cycles, so that complete energy optimization can be performed

- Developing a multiple input numerical optimization method that can be used for control and design

- Introducing a control method that reduces fuel consumption when compared to a traditional proportional controller on a military relevant scenario

- Showing the impact of the duty cycle on system energy used and design

\subsection{Future Work}

The future work could include running the the propulsion and actual electrical duty cycle three to four time consecutively to determine how the fuel used or battery capacity is effected. This type of simulation requires a great deal of computation power, therefore, some sort parallel computing effort would be recommended. Once the computing effort was solved, this could be part of a full parametric study on the shape of the electrical cycle, where the total energy would be constant, but the height and width of the step change would be altered. The results could impact the way electrical system architectures are designed. 



\section{References}

[1] F. An, F. Stodolsky, and J. Eberhardt, "Fuel and emission impacts of heavy hybrids," Argonne National Laboratory, vol. Report Number ANL/ES/CP98502, 1999 .

[2] D. Kramer and G. Parker, "Current state of military hybrid vehicle development," Int. J. Electric and Hybrid Vehicles, vol. 3, pp. 369-387, 2011.

[3] D. Rizzo and G. Parker, "State of charge optimization for military hybrid vehicle microgrids," in Proceedings of the IEEE SmartGrid Conference, February 2013.

[4] D. Rizzo and G.Parker, "Determining optimal state of charge for a military vehicle microgrid," International Journal of Powertrains, pp. 303-318, February 2014 .

[5] C. Chan, Y. Wong, A. Bouscayrol, and K. Chen, "Powering sustainable mobility: Roadmaps of electric, hybrid, and fuel cell vehicles," Proceedings of the IEEE, vol. 95, no. 4, April 2009.

[6] V. Dawood and A. Emadi, "Performance and fuel economy comparative analysis of conventional, hybrid and fuel cell heavy-duty transit buses," Proceeding of the Vehicular Technology Conference, vol. 5, pp. 3310-3315, October 2003.

[7] E. Rosenthal, "U.s. military orders less dependence on fossil fuels," New York Times, October 2010. 
[8] G. Khalil, "Challenges of hybrid electric vehicles for military applications," Proceedings of the IEEE Vehicle Power and Propulsion Conference, pp. 1-3, September 2009.

[9] D. Desmond, "Power on call how auxiliary power units can extend mission times in the abrams main battle tank," Accelerate Magazine Energy Efficiency Edition, pp. 37-38, June-August 2011.

[10] E. Shaffer, D. Massie, and J. Cross, "Power and energy architecture for army advanced energy initiative," Army Report No. ADM002075, November 2006.

[11] R. Lasseter, A. Akhil, C. Marnay, J. Stephens, J. Dagle, R. Guttromson, A. Mekipoulous, R. Yinger, and J.Eto, "Integration of distributed energy resources: The certs microgrid concept," U.S. Department of Energy and California Energy Commission, vol. LBNL-50829, 2002.

[12] R. Lasseter and P. Paigi, "Microgrid: A conceptual solution," IEEE 35th Annual Power Electronics Specialists Conference, vol. 6, pp. 4285-4290, January 2004.

[13] W. Kempton, J. Tomic, S. Letendre, A. Brooks, and T. Lipman, "Vehicle-togrid power: Battery, hybrid, and fuel cell vehicles as resources for distributed electric power in california," Institute for Transportation Studies Report No. UCD-ITS-RR-01-03, June 2001.

[14] G. Khalil, C. E. Allen, and M. Pozolo, "Hybrid-electric experimentation and assessment [hevea] program supports the army's need for increased power demand," Accelerate, pp. 4-7, 2009.

[15] C. Williams, "Hybrid-electric (he) drive knowledge expanded through validation testing," Accelerate, pp. 20-21, July-September 2009.

[16] J. Hodgson, W. Hamel, C. Rutherford, and J. Armfield, "Design of a low production cost hybrid hmmwv," in Proceedings of IEEE Digital Avionics Systems Conference, vol. 2, October 1998, p. I46/1.

[17] M. Freeman, "Hybrid power an enabling technology for future combat systems," Proceedings of the IEEE Pulsed Power Conference, vol. 1, pp. 17-22, June 1999. 
[18] R. Crow and S. Cortese, "Dual use hybrid technology for the 21st century truck program," SAE Paper No. 2000-01-3421, December 2000.

[19] C. Johnson and L. Dueck, "Hybrid electric systems for 20-ton platform," SAE Paper No. 2001-01-2773, November 2001.

[20] J. Ernat, W. Harris, and T. Trzaska, "Advanced hybrid electric drive (ahed) technology demonstrator," SAE Paper No. 2001-01-2774, November 2001.

[21] M. A. Kluger, D. Szkubiel, and E. A. Bass, "A dual-use hybrid electric command and control vehicle," SAE Paper No. 2001-01-2775, November 2001.

[22] H. Almand, W. Doolittle, and G. Klees, "Hybrid propulsion truck platform dually developed by the u.s. army and daimlerchrysler," SAE Paper No. 200101-2794, 2001.

[23] J. M. Paschen and C. Johnson, "Shared technology/shared cost, the evaluation of the allison ep-50 electric drive for military and commercial vehicles," $S A E$ Paper No. 2003-01-0271, March 2003.

[24] P. DiSante, "Hybrid drive partnerships keep the army on the right road," $R D E$ COM Magazine, June 2003.

[25] W. G. Harris and D. L. Ventimeglia, "Will hybrid electric propulsion drive future combat systems," SAE Paper No. 2002-01-03052, March 2002.

[26] K. Chung, A. Reid, D. Fatemi, and G. Fryer, "Performance based purchase description hybrid electric high mobility multipurposed wheeled vehicle," Proceedings of the 8th World Congress on Computational Mechanics (WCCM8), 5th European Congress on Computational Methods in Applied Sciences and Engineering (ECCOMAS 2008), June 2008.

[27] K. Osborn, "3 firms win jltv contract," DefenseNews:http://www.defensenews. com/story.php $\mathrm{i}=3795022 \& \mathrm{c}=\mathrm{AME} \& \mathrm{~s}=\mathrm{LAN}$.

[28] P. Mcleary, "Army taking another look at jltv," AviationWeek: http://www.aviationweek.com/aw/generic/story_generic.jsp?channel= aerospacedaily\&id=news/asd/2010/03/03/07.xml. 
[29] C. Johnson, "Fuel savings design and engineering drive the army's fed program," Accelerate: FED Special Edition, pp. 4-9, 2010.

[30] C. Williams, "Modeling the fed-new concepts, new techniques," Accelerate: FED Special Edition, pp. 10-13, 2010.

[31] M. Seaton and J. Gardini, "Systems engineering approach crucial for the fed vehicles," Accelerate: FED Special Edition, pp. 22-27, 2010.

[32] P. Luskin and R. Berlin, "Systems engineering methodology for fuel efficiency and its application to the tardec fuel efficient demonstrator (fed) program," Proceedings of the NDIA Ground Systems Engineering and Technology Symposium: Systems Engineering Mini-Symposium, August 2010.

[33] E. P. Agency, "Vehicle weight classifications emission standards reference guide," http://www.epa.gov/otaq/standards/weights.htm.

[34] F. An, F. Todolsky, A. Vyas, R. Cuenca, and J. Eberhardt, "Scenario analysis of hybrid class 3-7 heavy vehicles," SAE Paper No. 2000-01-0908, March 2000.

[35] A. Emadi, K. Rajashekara, S. S. Williamson, and S. M. Lukic, "Topological overview of hybrid electric and fuel cell vehicular power system architectures and configurations," IEEE Transactions on Vehicular Technology, vol. 54, no. 3, pp. 763-770, May 2005.

[36] M. Ehsani, Y. Gao, and J. M. Miller, "Hybrid electric vehicles: Architecture and motor drives," Proceedings of IEEE, vol. 95, no. 4, pp. 719-728, April 2007.

[37] C. Chan, "The state of the art of electric, hybrid and fuel cell vehicles," Proceedings of IEEE, vol. 95, no. 4, pp. 704-718, April 2007.

[38] J. Stecki and P. Matheson, "Advances in automotive hybrid drives," Proceedings of the 6th JFPS International Symposium on Fluid Power, TSUKUBA, November 2005.

[39] "Drive cycle data," http://www.epa.gov/nvfel/testing/dynamometer.htm.

[40] R. Kruse and T. A. huls, "Development of the federal urban driving schedule," in Proceedings of the SAE Automobile Engineering Meeting, Paper No. 730533, August 1973. 
[41] E. Milikins and H. Watson, "Comparison of urban driving patterns," SAE Paper No. 830939, March 1983.

[42] J. Crauser, M. Maurin, and R. Joumard, "Representative Kinematic Sequences for the Road Traffic in France," SAE Paper No. 890875, March 1989.

[43] M. Montazeri-Gh and M. Naghizadeh, "Development of Car Drive Cycle for Simulation of Emissions and Fuel Economy," in Proceedings of the 15th European Simulation Symposium, August 2003.

[44] R. A. Barnitt and J. Gonder, "Drive Cycle Analysis, Measurement of Emissions and Fuel Consumption of a PHEV School Bus," SAE Paper No. 2011-01-0863, April 2011.

[45] Z. Ivani, "Data Collection and Development of New York City Refuse Truck Duty Cycle," SAE Paper No. 200\%-01-4118, Feburary 2007.

[46] M. Surcel, J. Michaelsen, and Y. Provencher, "Development of a Fuel Consumption Test Procedure for Representative Urban Duty Cycles," SAE Paper No. 2011-01-2291, September 2011.

[47] M. Andre, R. Joumard, A. Hickman, and D. Hassel, "Actual car use and operating conditions as emission parameters: derived urban driving cycles," The Science of the Total Environment, vol. 146-147, pp. 225-233, 1994.

[48] M. Andrea, R. Joumard, A. Hickman, and D. Hassel, "Drive Cycle for Emissions Measurements Under European Conditions," SAE Paper No. 950962, April 1995.

[49] B. Adornato, R. Patil, Z. Filipi, Z. Baraket, and T. Gordon, "Characterizing naturalistic driving patterns for Plug-in Hybrid Electric Vehicle analysis," in Proceedings of the IEEE Vehicle Power and Propulsion Conference, September 2009, pp. 655-660.

[50] Q. Wang, H. Huo, Z. Yao, and Q. Zhang, "Characterization of vehicle driving patterns and development of driving cycles in Chinese cities," Transportation Research Part D: Transport and Environment, vol. 13, pp. 289-297, July 2008. 
[51] H. Wi, J. Park, J. Lee, W. Kim, and Y. Kim, "Development of a City Bus Driving Cycle in Seoul Based on the Actual Patterns of Urban Bus Driving," SAE Paper No. 2009-01-2914, April 2009.

[52] M. Rapone, L. D. Ragione, and G. Meccariello, "Characterization of Real World Bus Driving Behavior for Emission Evaluation," SAE Paper No. 2007-24-0112, September 2007.

[53] G. Perkins, "Analytic Process to Develop a Local Truck Driving Cycle," SAE Paper No. 821256, March 1982.

[54] H. Bruneel, "Heavy Duty Testing Cycles Development: A New Methodology," SAE Paper No. 2000-01-1860, June 2000.

[55] N. Dembski, Y. Guezennec, and A. Soliman, "Analysis and Experimental Refinement of Real-World Driving Cycles," SAE Paper No. 2002-01-0069, April 2002.

[56] Q. Gong, S. Midlam-Mohler, V. Marano, and G. Rizzoni, "An Iterative Markov Chain Approach for Generating Vehicle Driving Cycles," SAE Paper No. 201101-0880, April 2011.

[57] R. Bata, Y. Yacoub, W. Wang, and D. Lyons, "Heavy Duty Testing Cycles: Survey and Comparison," SAE Paper No. 942263, November 1994.

[58] R. Rykowski, E. Nam, and G. Hoffman, "On-road Testing and Characterization of Fuel Economy of Light-Duty Vehicles," SAE Paper No. 2005-01-0677, May 2005.

[59] M. OKeefe, A. Simpson, K. Kelly, and D. Pedersen, "Duty Cycle Characterization and Evaluation Towards Heavy Hybrid Vehicle Applications," SAE Paper No. 2007-01-0302, May 2007.

[60] Z. Zou, S. Davis., K. Beaty, M. O'Keefe, T. Hendricks, R. Rehn, S. Weissner, and V. Sharma, "A New Composite Drive Cycle for Heavy-Duty Hybrid Electric Class 4-6 Vehicles," SAE Paper No. 2004-01-1052, April 2004.

[61] M. Brudnak, M. Pozolo, M. McGough, T. Mortsfield, A. Shvartsman, and R. Romano, "Motion based simulation of a hybrid-electric hmmwv for economy improvements," SAE Paper No. 2008-01-0775, April 2008. 
[62] N. Dembski, G. Rizzoni, and A. Soliman, "Development and Application of Military Wheeled Vehicle Driving Cycle Generator," SAE Paper No. 2005-013560, April 2005.

[63] A. Malikopoulos, Z. Filipi, and D. Assanis, "Simulation of an integrated starter alternator (isa) system for the hmmwv," SAE Paper No. 2006-01-0442, April 2006.

[64] A. I. Antoniou, J. Komyathy, J. Bench, and A. Emad, "Modeling and simulation of various hybrid-electric configurations of the high-mobility multipurpose wheeled vehcile (hmmwv)," IEEE Transactions on Vehicular Technology, vol. 56, no. 2, pp. 459-465, March 2007.

[65] D. Milner, J. Goodell, W. Smith, J. Tzau, M. Pozolo, and J. Ueda, "Power system design optimization for tactical wheeled vehicles," Proceedings of the EVS24 International battery Hybrid and Fuel Cell Electric Vehicle Symposium, May 2009.

[66] Z. Filipi, H. Fathy, J. Hagena, A. Knafl, R. Ahlawat, J. Liu, D. Jung, D. Assanis, H. Peng, and J. Stein, "Engine- in-the-loop testing for evaluating hybrid propulsion concepts and transient emissions: Hmmwv case study," SAE Paper No. 2006-01-0443, April 2006.

[67] M. Gokasan, S. Bogosyan, and D. Goering, "Sliding mode based powertrain control for efficiency improvement in series hybrid-electric vehicles," IEEE Transactions on Power Electronics, vol. 12, no. 3, May 2006.

[68] H. Bargar, J. Li, D. Goering, and J. Lee, "Modeling and verification of hybrid electric hmmwv performance," Proceeding of the IEEE Industrial Electronics Society 29th Annual Conference, vol. 1, p. 939, November 2003.

[69] Y. J. Kim and Z. Filipi, "Simulation study of a series hydraulic hybrid propulsion system for a light truck," SAE Paper No. 2007-01-4151, 2007.

[70] Z. Filipi, L. Louca, A. Stefanopoulou, J. Pukrushpan, B. Kittirungsi, and H. Peng, "Fuel cell apu for silent watch and mild electrification of a medium tactical truck," SAE Paper no. 2004-01-1477, March 2004. 
[71] Z. Filipi, L. Louca, B. Daran, C. Lin, U. Yildir, B. Wu, M. Kokkolaras, D. Assanis, H. Peng, P. Papalambros, J. Stein, S. Szkubiel, and R. Chapp, "Combined optimization of design and power management of the hydraulic hybrid propulsion system for the 6x6 medium truck," Int. J. of Heavy Vehicle Systems, vol. 11, no. Nos. 3/4, 2004.

[72] C. Liang, W. Weihua, and W. Qingnian, "Energy management strategy and parametric design for hybrid electric military vehicle," SAE Paper No. 200301-0086, March 2003.

[73] G. Rizzoni, J. Johnson, A. Soliman, C. Huber, C. Cantemir, N. Dembski, P. Pisu, D. Mikesell, L. Serrao, J. Russell, and M. Caroll, "Modeling, simulation, and concept design for hybrid-electric medium-size military trucks," Proceedings of SPIE, vol. 5805, 2005.

[74] B. Surampudi, A. Nedungadi, G. Ostrowski, A. Montemayor, and H. Gruenewald, "Design and control considerations for a series heavy duty hybrid hydraulic vehicle," SAE Paper no. 2009-01-2717, 2009.

[75] P. Matheson and J. Stecki, "Development and simulation of a hydraulic-hybrid powertrain for use in commercial heavy vehicles," SAE 2003-01-3370, November 2003.

[76] J. Whitefoot, A. Mechtenberg, D. Peters, and P. Papalambros, "Optimal component sizing and forward-looking dispatch of an electrical microgrid for energy storage planning," Proceedings of the ASME International Design Engineering Technical Conference and Computers and Information in Engineering Conference, Paper No. DETC2011-485513, August 2011.

[77] D. Peters, A. Mechtenberg, J. Whitefoot, and P. Papalambros, "Model predictive control of a microgrid with plug-in vehicles: Error modeling and the role of prediction horizon," Proceedings of the ASME Dynamic Systems and Control Conference, Paper No. DSCC2011-6030, November 2011.

[78] T. Ersal, C. Ahn, I. Hiskens, H. Peng, A. Stefanopoulou, and J. Stein, "On the effect of dc source voltage on inverter-based frequency and voltage regulation in a military microgrid," Proceedings of the2012 American Control Conference (ACC), June 2012. 
[79] T. Ersal, C. Ahn, I. Hiskens, H. Peng, and J. Stein, "Impact of controlled plugin evs on microgrids: A military microgrid example," Proceedings of the Power and Energy Society General Meeting, IEEE, pp. 1-7, July 2011.

[80] O. R. N. Laboratory, "Evaluation of the 2010 toyota prius hybrid synergy drive system," http://info.ornl.gov/sites/publications/files/Pub26762.pdf.

[81] J. Liu, H. Peng, and Z. Filipi, "Modeling and analysis of the toyota hybrid system," Proceedings of the 2005 IEEE/ASME International Conference of Advanced Intelligent Mechatronics, July 2005.

[82] J. Liu and H. Peng, "Modeling and control of a power-split hybrid vehicle," IEEE Transactions on Control Systems Technology, vol. 16, November 2008.

[83] T. M. Corporation, "Toyota hybrid system ths ii," http://www.evworld.com/ library/toyotahs2.pdf.

[84] T. Mathworks, "Fmincon r2012a documentation," http://http://www. mathworks.com/help/toolbox/optim/ug/fmincon.html.

[85] E. Kreysig, Advanced Engineering Mathematics. John Wiley and Sons, Inc., 1999.

[86] D. Luenberger, Optimization by Vector Space Methods. John Wiley and Sons Inc, 1969.

[87] G. Franklin, J. Powell, and A. Emami-Naeini, Feedback Control of Dynamic Systems. Pearson Higher Education, 2010.

[88] ATPD 2335, "Performance based purchase description hybrid electric high mobility multipurposed wheeled vehicle," U.S. Army Tank Automotive Research, Development and Engineering Center, Tech. Rep., May 2003.

[89] M. Ducusin, S. Gargies, and C. Mi, "Modeling of a series hybrid electric highmobility multipurpose wheeled vehicle," IEEE Transactions on Vehicular Technology, vol. 56, no. 2, March 2007. 
[90] N. Michelena, L. Louca, M. Kokkolaras, C.C. Lin, D. Jung, Z. Filipi, D. Assanis, P. Papalambros, H. Peng, J. Stein, and M. Feury, "Design of an advanced heavy tactical truck: A target cascading case study," SAE Paper No. 2001-01-2793, November 2001.

[91] D. Lucia, P. Beran, and W. Silva, "Reduced-order modeling: new approaches for computational physics," Progress in Aerospace Sciences, vol. 40, pp. 51-117, 2003.

[92] G. Kerschen abd J. Golinval, A. Vakakis, and L. Bergman, "The method of proper orthogonal decomposition for dynamical characterization and order reduction of mechanical systems: An overview," Nonlinear Dynamics, vol. 40, pp. 147-169, 2005.

[93] P. Glosmann and E. Kreuzer, "The method of proper orthogonal decomposition for dynamical characterization and order reduction of mechanical systems: An overview," Nonlinear Dynamics, vol. 41, pp. 111-128, 2005.

[94] C. Frouzakis, Y. Kevrekids, J. Lee, and A. Alonso, "Proper orthogonal decomposition of direct numerical simulation data: Data reduction and observer construction," Proceedings of the Combustion Institute, vol. 41, pp. 75-81, 2005.

[95] M. Schetzen, THe Volterra and Wiener Theories of Nonlinear Systems. Krieger, 1989.

[96] L. Chua and Y. Yang, "Nonlinear oscillation via volterra series," IEEE Trans. Circuits and Systems, vol. 29, pp. 150-168, 1982.

[97] A. Zhu, J. Pedro, and T. Cunha, "Pruning the volterra series for behavioral modeling of power amplifiers using physical knowledge," IEEE Trans. Microwave Theory and Techniques, vol. 55, pp. 813-821, 2007.

[98] H. Stalford, W. Baumann, F. Garrett, and T. Herdman, "Accurate modeling of nonlinear systems using volterra series submodels," Proceedings of the American Controls Conference, June 1987.

[99] D. Lucia, P. Beran, and W. Silva, "Reduced order modeling: new approaches in computational physics," Progress in Aerospace, vol. 40, pp. 51-117, 2004. 
[100] M. Reed and M. Hawksford, "Identification of discrete volterra series using maximum length sequences," IEEE Proc. Circuits Devices Syst., vol. 143, pp. 241-248, 1987.

[101] A. Kaizer, "Modeling of the nonlinear response of an electrodynamic loudspeaker by a volterra series expansion," J. Audio Eng. Soc., vol. 35, pp. 421-432, 1987.

[102] P. Alper, "A consideration of the discrete volterra series," IEEE Transactions on Automatic Control, vol. 10, pp. 322-327, 1965. 



\section{Appendix A}

\section{Code}

cost calculation:

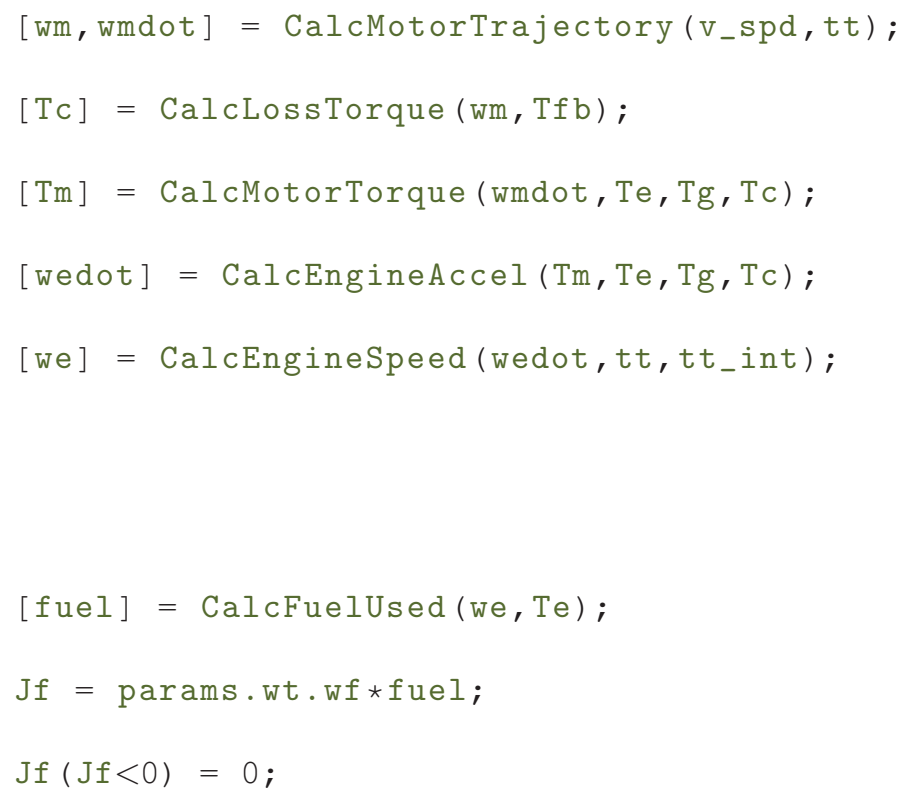




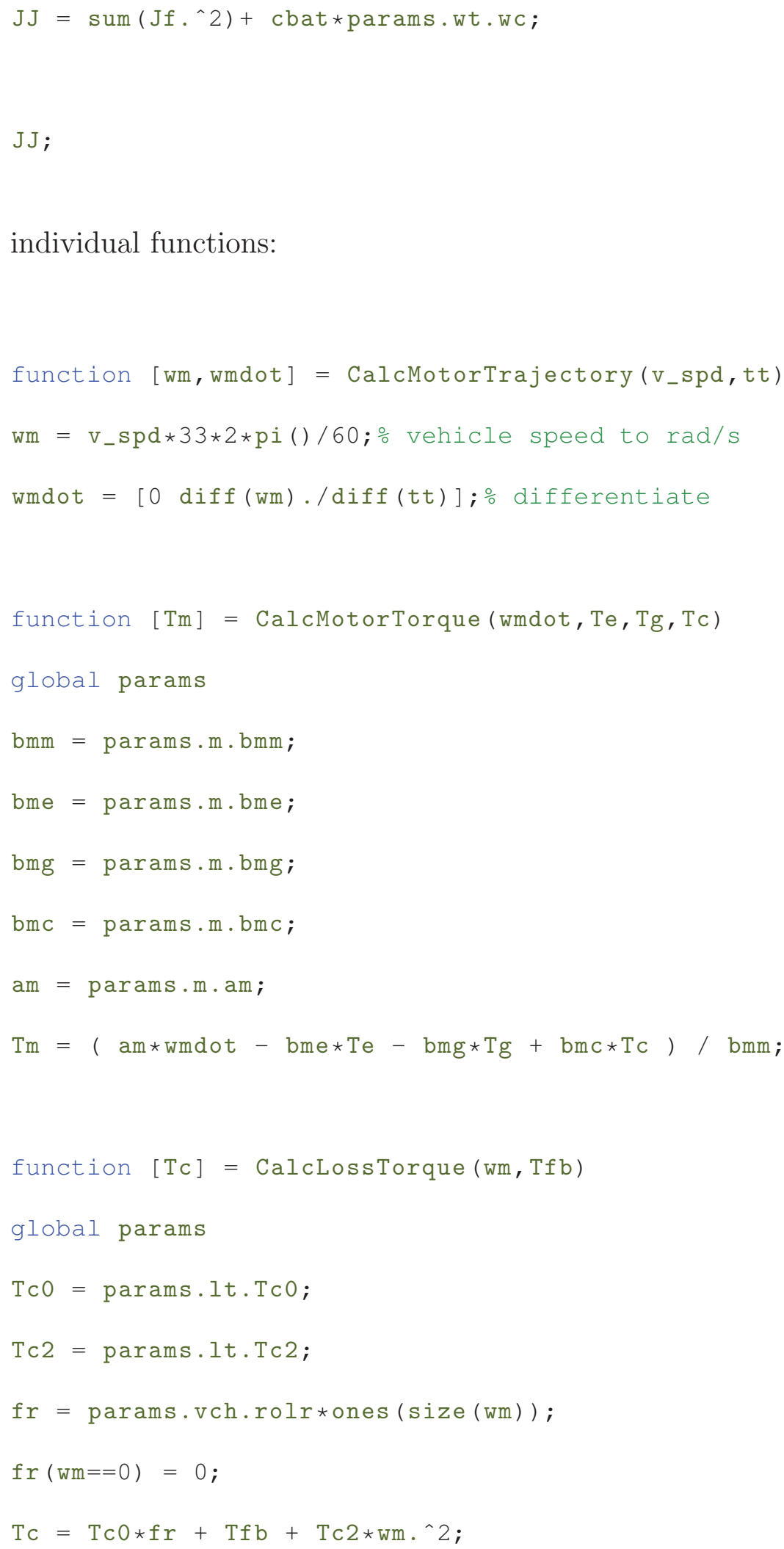




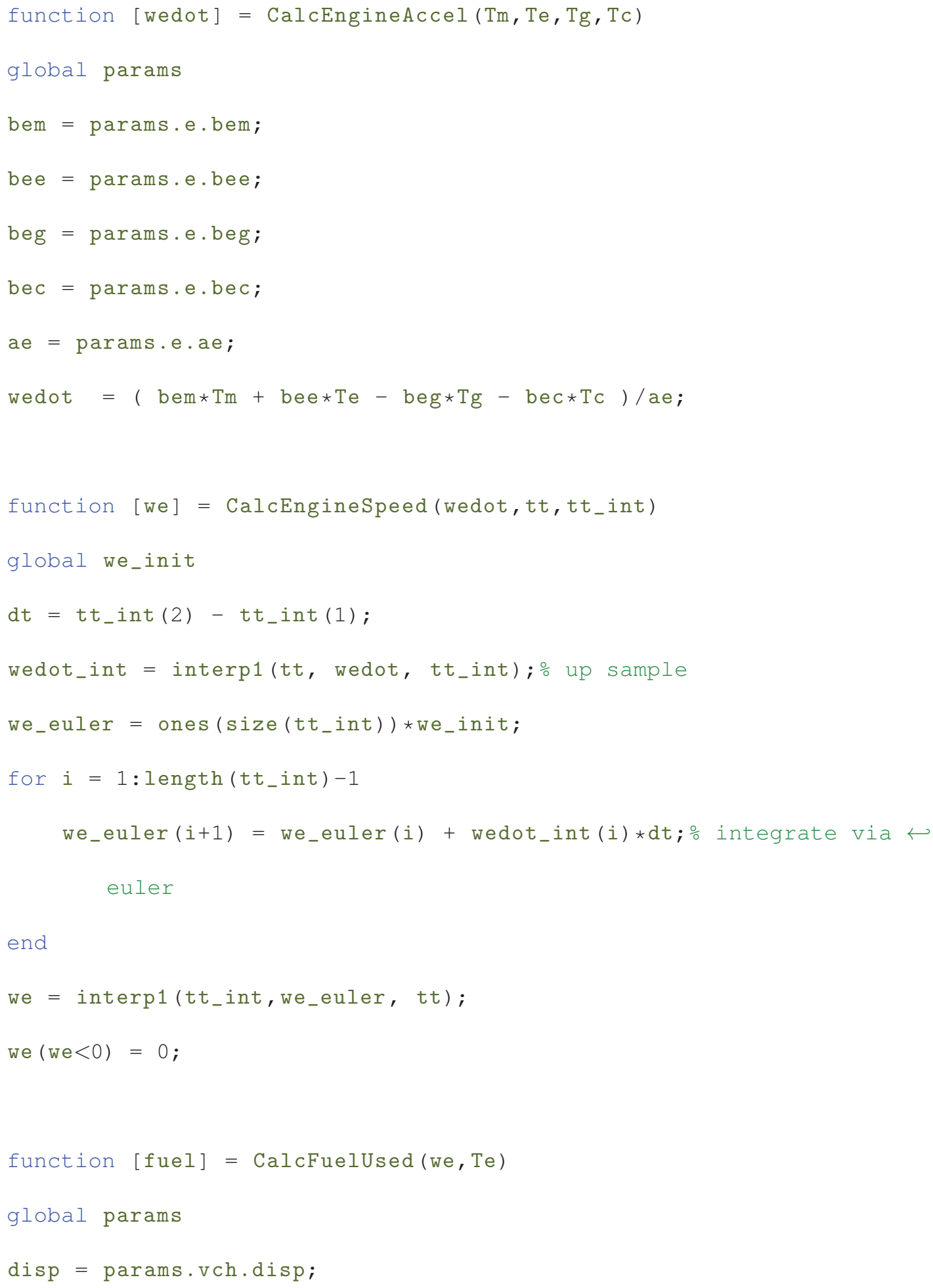




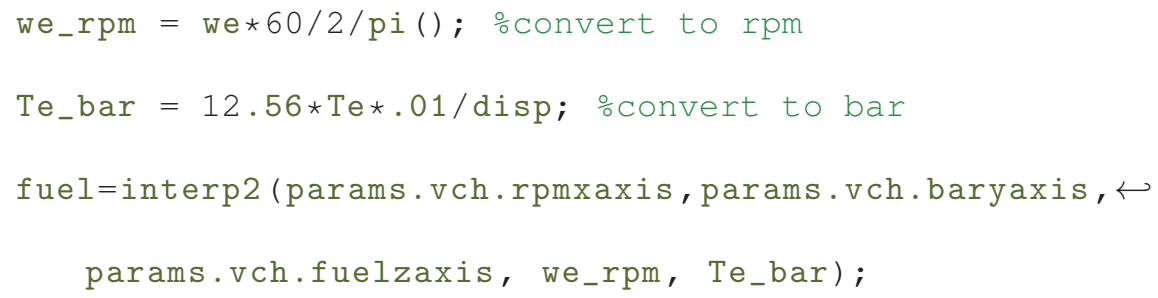

constraint calculation:

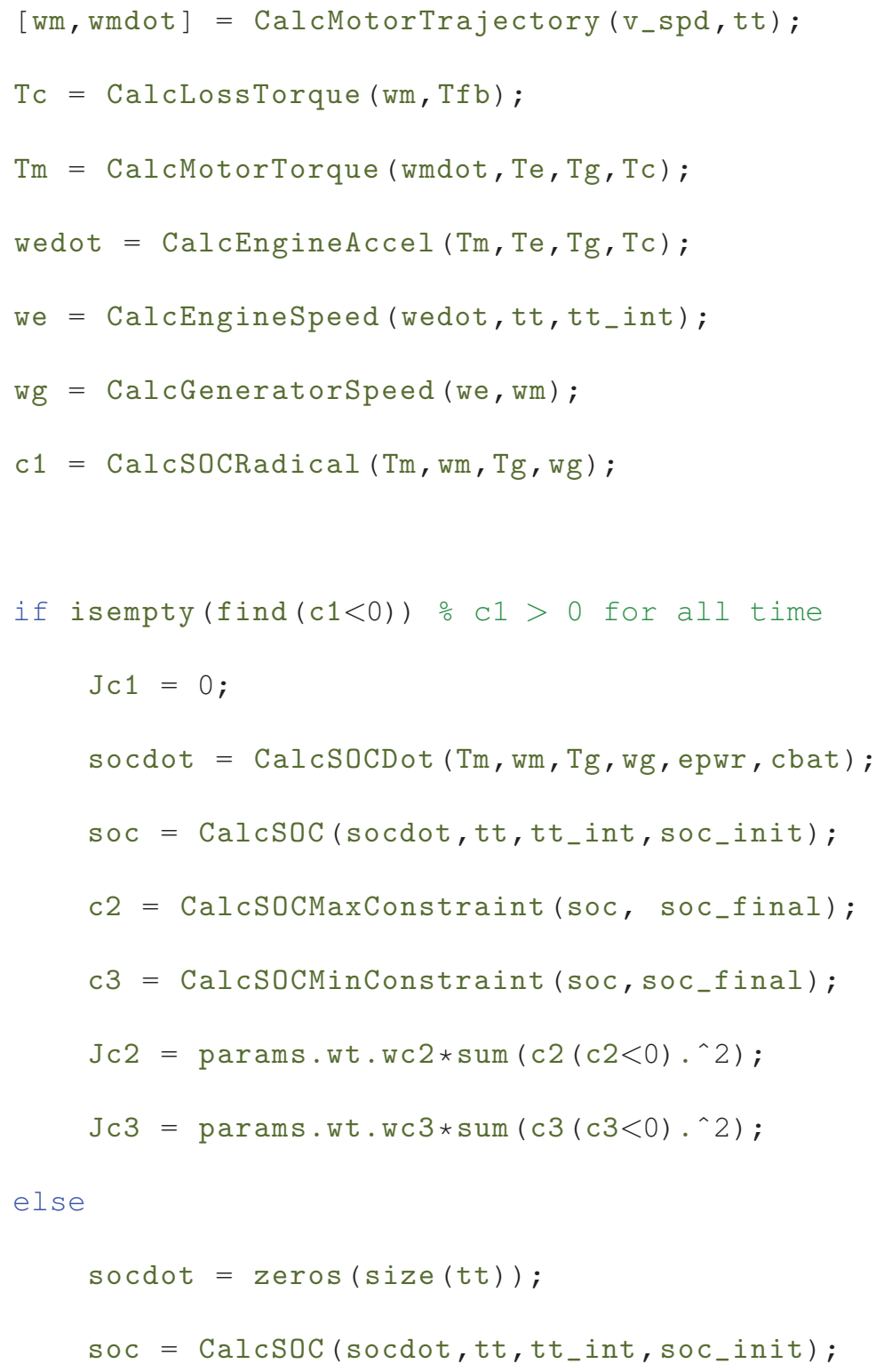




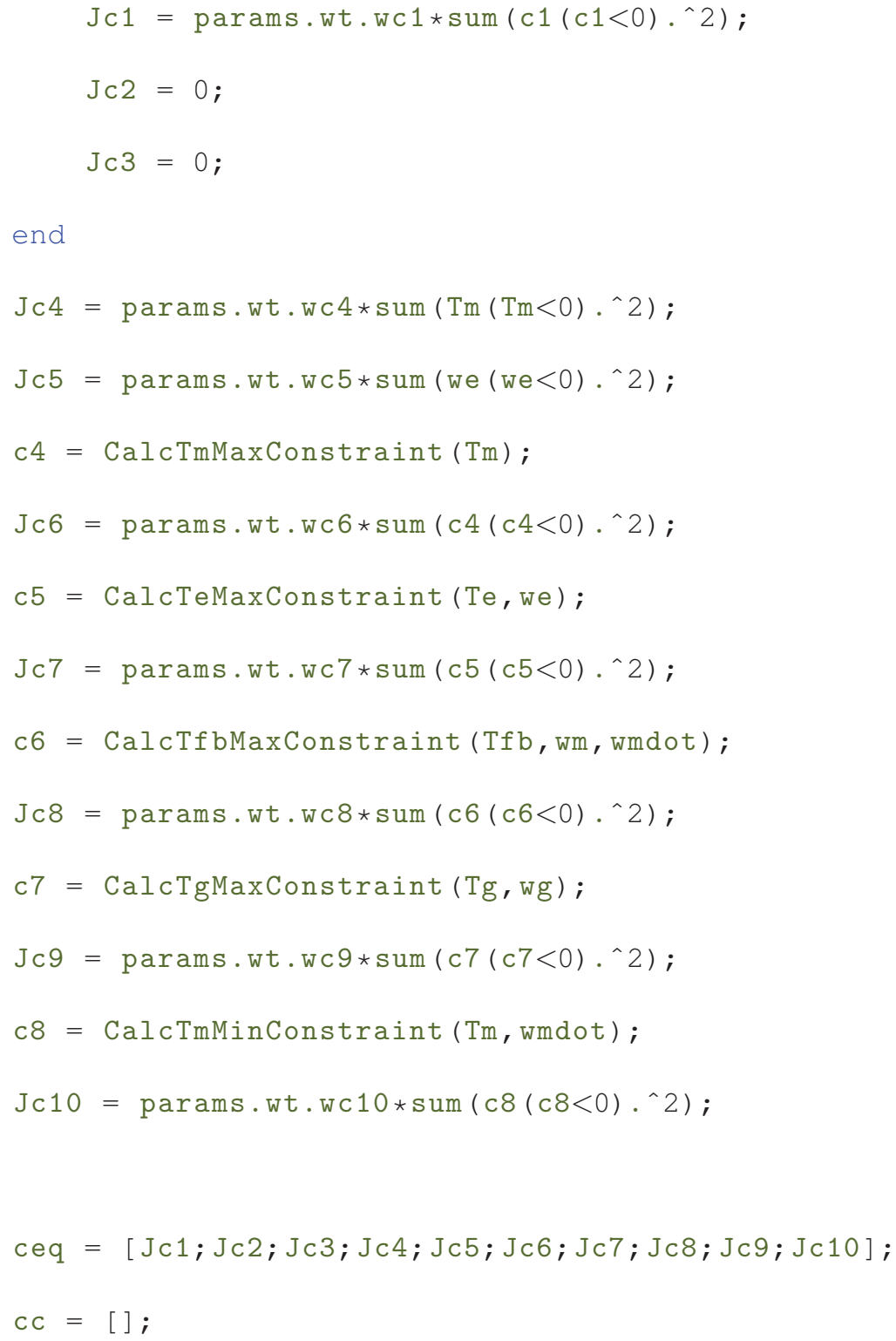

Individual functions:

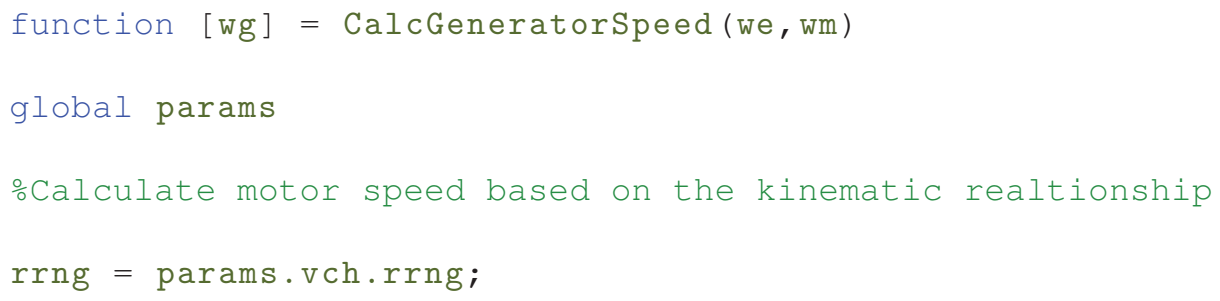




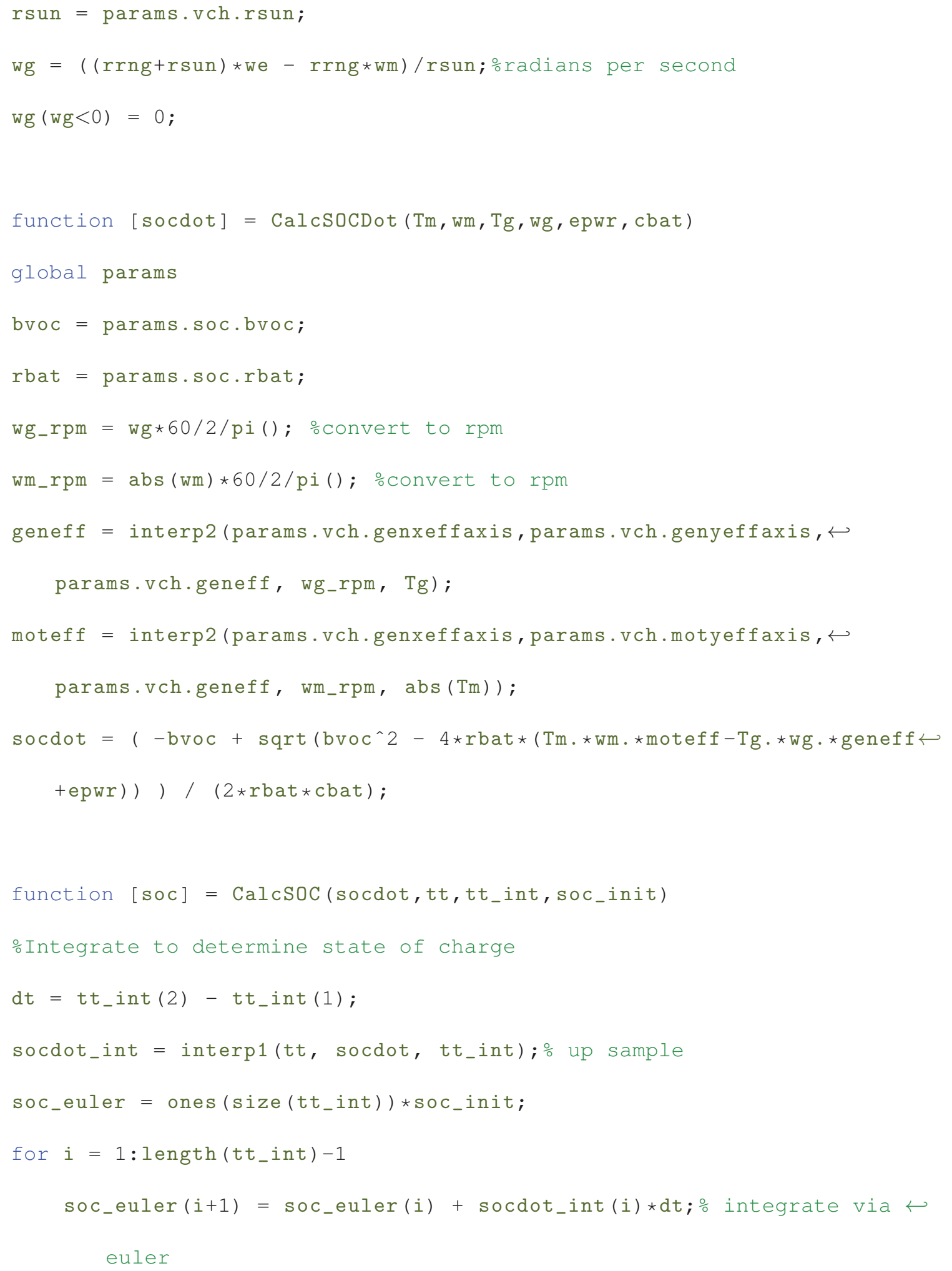




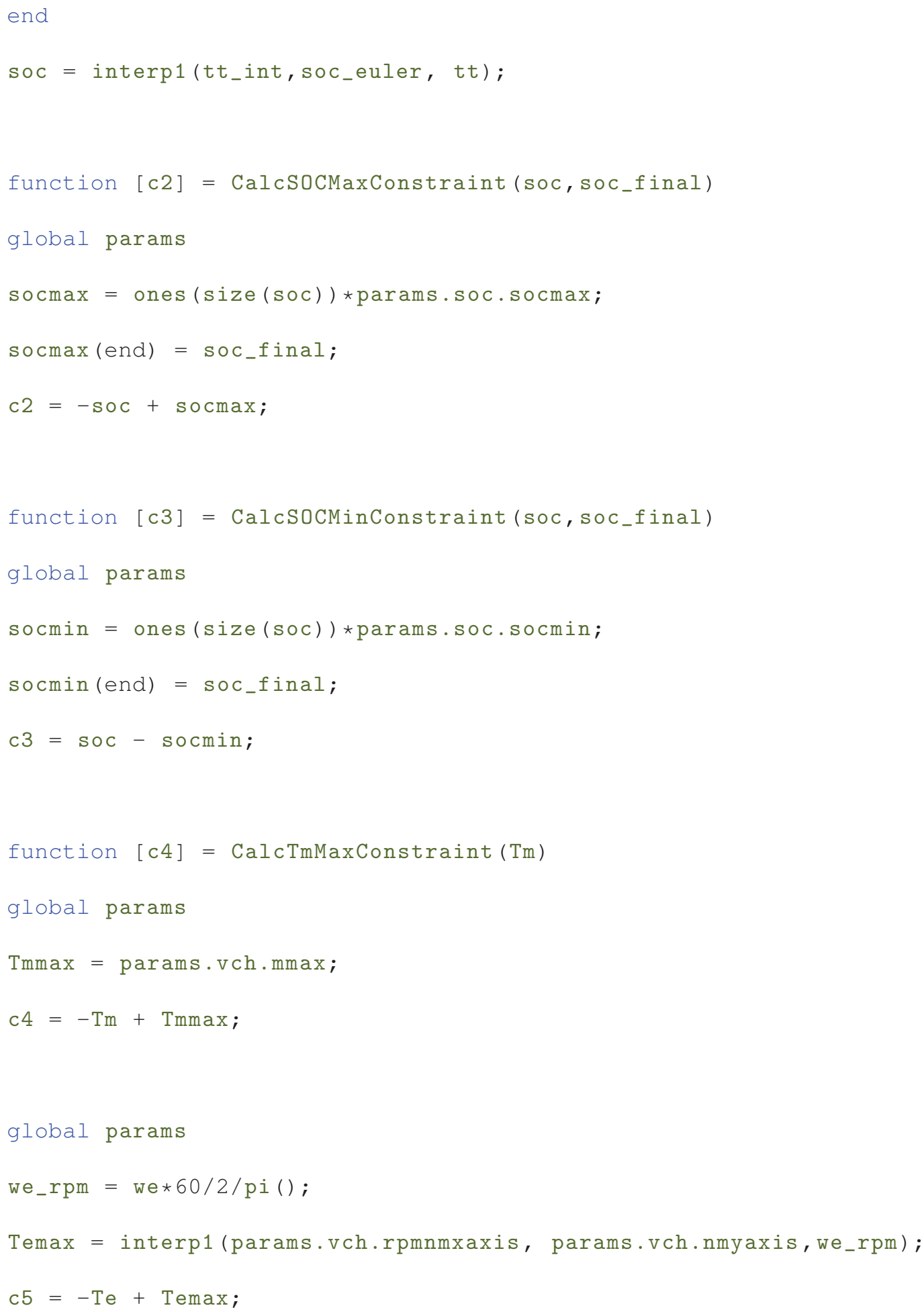




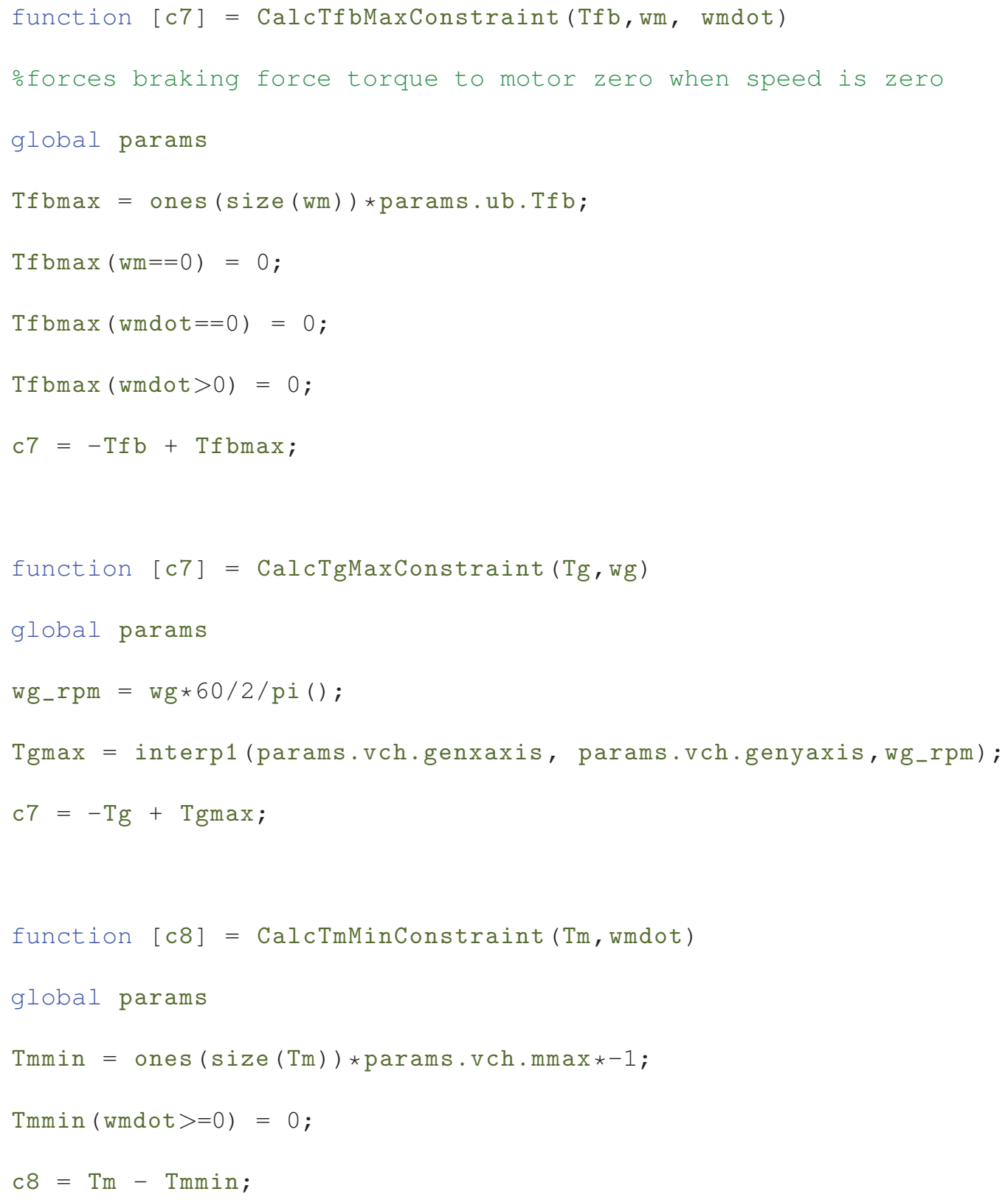




\section{Appendix B}

\section{Simplified Vehicle Model}

\section{Supporting Equations}

$$
B=\left[\begin{array}{ccc}
\frac{R}{\mathrm{I}_{\mathrm{ep}}}+\frac{2 S}{\mathrm{I}_{\mathrm{ep}}}+\frac{S^{2}}{\mathrm{I}_{\mathrm{ep}} R}+\frac{S^{2}}{\mathrm{I}_{\mathrm{gp}} R} & \frac{R}{\mathrm{I}_{\mathrm{ep}}}+\frac{S}{\mathrm{I}_{\mathrm{ep}}} & -\frac{S}{\mathrm{I}_{\mathrm{gp}}} \\
\frac{K R}{\mathrm{I}_{\mathrm{vp}}} & \frac{S^{2}}{\mathrm{I}_{\mathrm{gp}} R+\mathrm{I}_{\mathrm{gp}} S}+\frac{K R^{2}}{\mathrm{I}_{\mathrm{vp}} R+\mathrm{I}_{\mathrm{vp}} S} & -\frac{S}{\mathrm{I}_{\mathrm{gp}}} \\
-\frac{4 \mathrm{R}_{\mathrm{batt}} \mathrm{w}_{\mathrm{r}}}{\mathrm{n}_{\mathrm{m}} k} & 0 & \mathrm{p}
\end{array}\right]
$$

where,

$$
\mathrm{p}=4 \mathrm{R}_{\text {batt }} \mathrm{n}_{\mathrm{g}}{ }^{k} \mathrm{w}_{\mathrm{e}}-\frac{4 \mathrm{R}_{\text {batt }} \mathrm{n}_{\mathrm{g}}{ }^{k} \mathrm{w}_{\mathrm{r}}}{S}+\frac{4 R \mathrm{R}_{\text {batt }} \mathrm{n}_{\mathrm{g}}{ }^{k} \mathrm{w}_{\mathrm{e}}}{S}
$$




$$
f(x)=\left[\begin{array}{c}
\frac{C R}{\mathrm{I}_{\mathrm{ep}} K}+\frac{2 C S}{\mathrm{I}_{\mathrm{ep}} K}+\frac{C S^{2}}{\mathrm{I}_{\mathrm{ep}} K R}+\frac{C S^{2}}{\mathrm{I}_{\mathrm{gp}} K R}+\frac{\mathrm{I}_{\mathrm{vp}} R \dot{w}_{r}}{\mathrm{I}_{\mathrm{ep}} K}+\frac{2 \mathrm{I}_{\mathrm{vp}} S \dot{w}_{r}}{\mathrm{I}_{\mathrm{ep}} K}+\frac{\mathrm{I}_{\mathrm{vp}} S^{2} \dot{w}_{r}}{\mathrm{I}_{\mathrm{ep}} K R}+\frac{\mathrm{I}_{\mathrm{vp}} S^{2} \dot{w}_{r}}{\mathrm{I}_{\mathrm{gp}} K R} \\
R \dot{w}_{e}+S \dot{w}_{e}+\frac{C R}{\mathrm{I}_{\mathrm{vp}}}+\frac{\mathrm{I}_{\mathrm{ep}} S^{2} \dot{w}_{e}}{\mathrm{I}_{\mathrm{gp}} R+\mathrm{I}_{\mathrm{gp}} S}+\frac{\mathrm{I}_{\mathrm{ep}} K R^{2} \dot{w}_{e}}{\mathrm{I}_{\mathrm{vp}} R+\mathrm{I}_{\mathrm{vp}} S} \\
\mathrm{C}_{\mathrm{batt}} S C^{2}+2 \mathrm{~V}_{\mathrm{oc}} \mathrm{C}_{\mathrm{batt}} S \dot{O} C
\end{array}\right]
$$




\section{Appendix C}

\section{Mupad Code for Linearization}

The following code was used to linearize the vehicle model equations through Taylor series.

// Dynamic equations

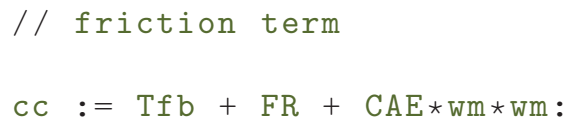




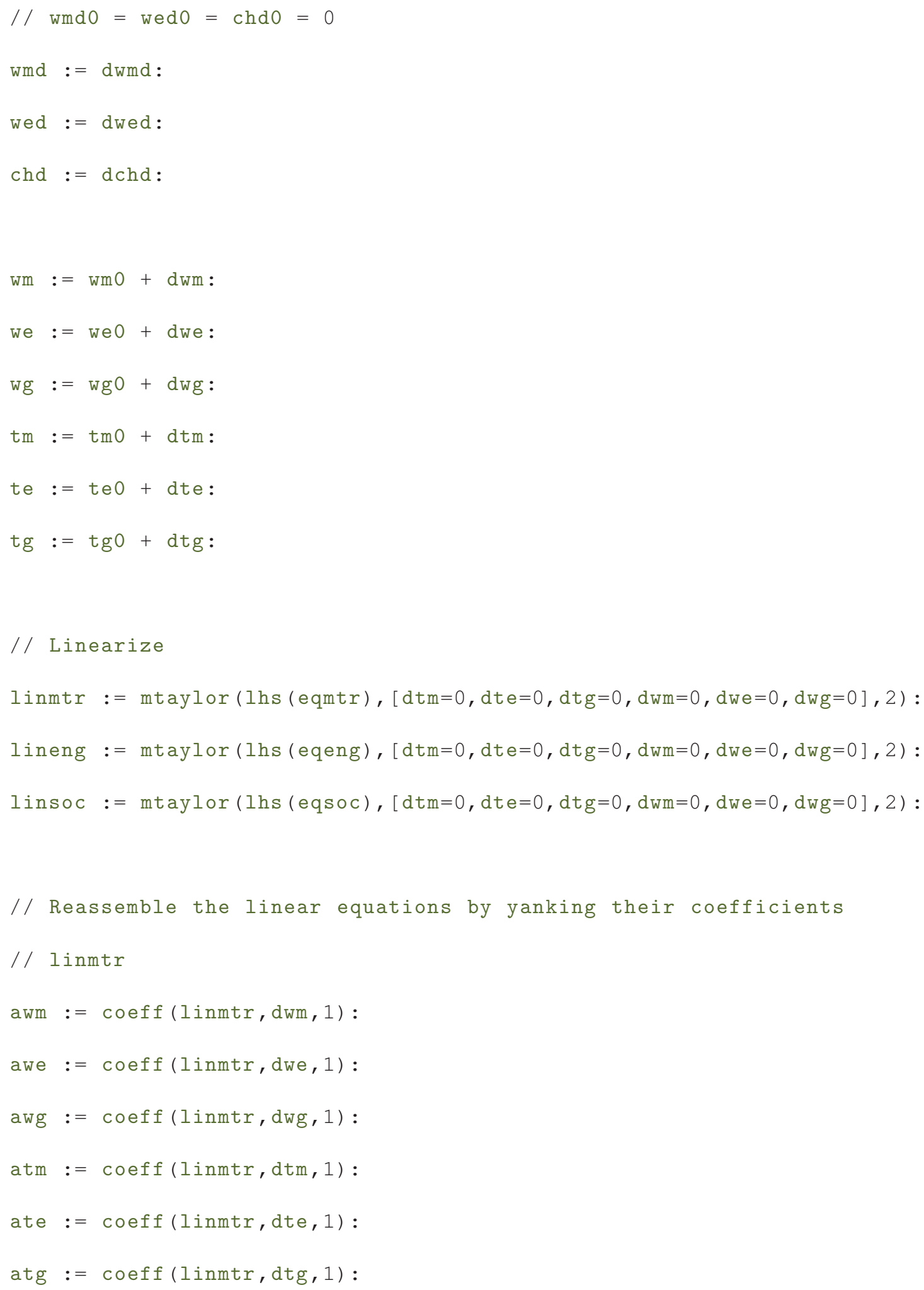




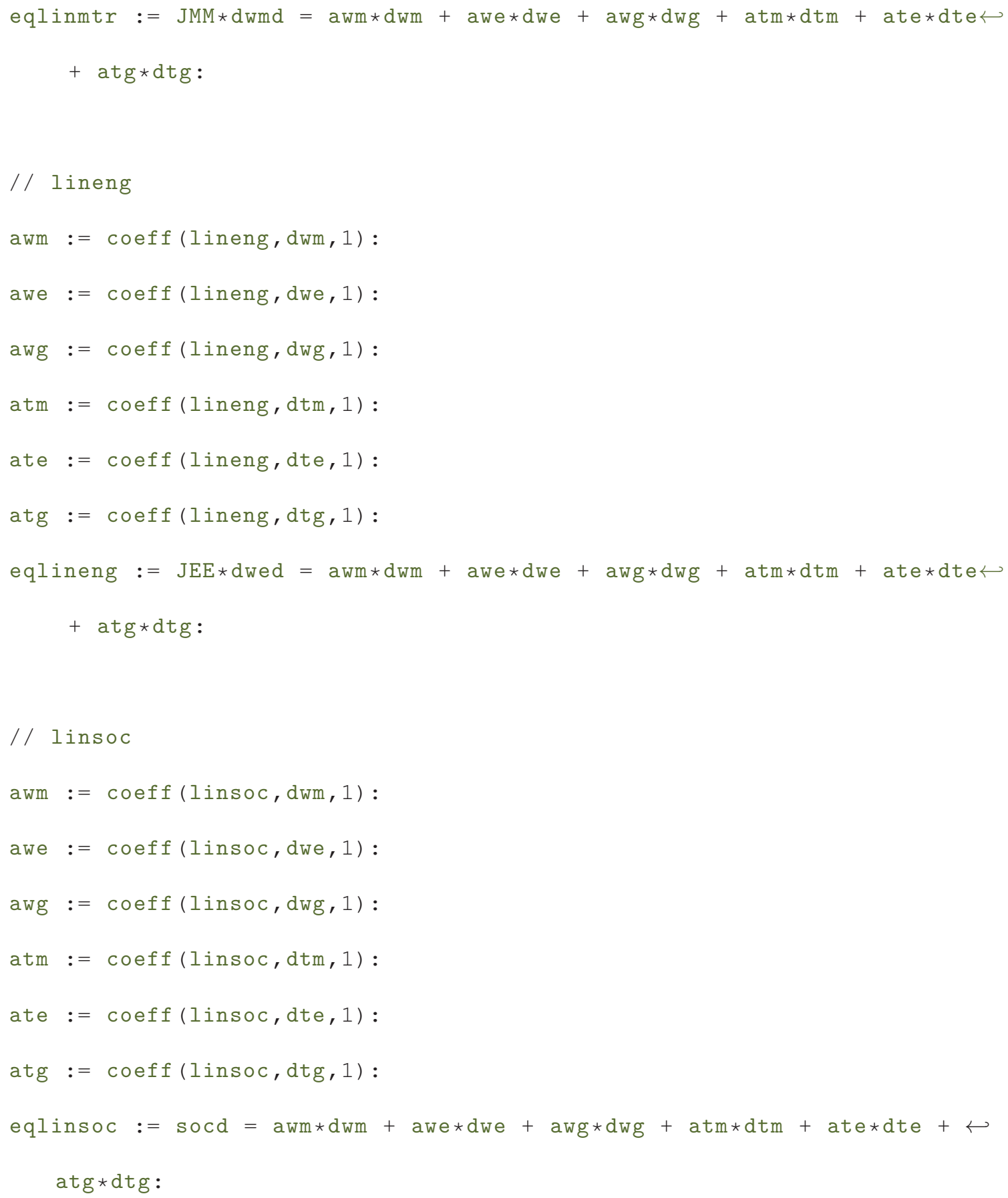





\section{Appendix D}

\section{Supporting Figures for Control}

The following figures support the real time optimization control results.

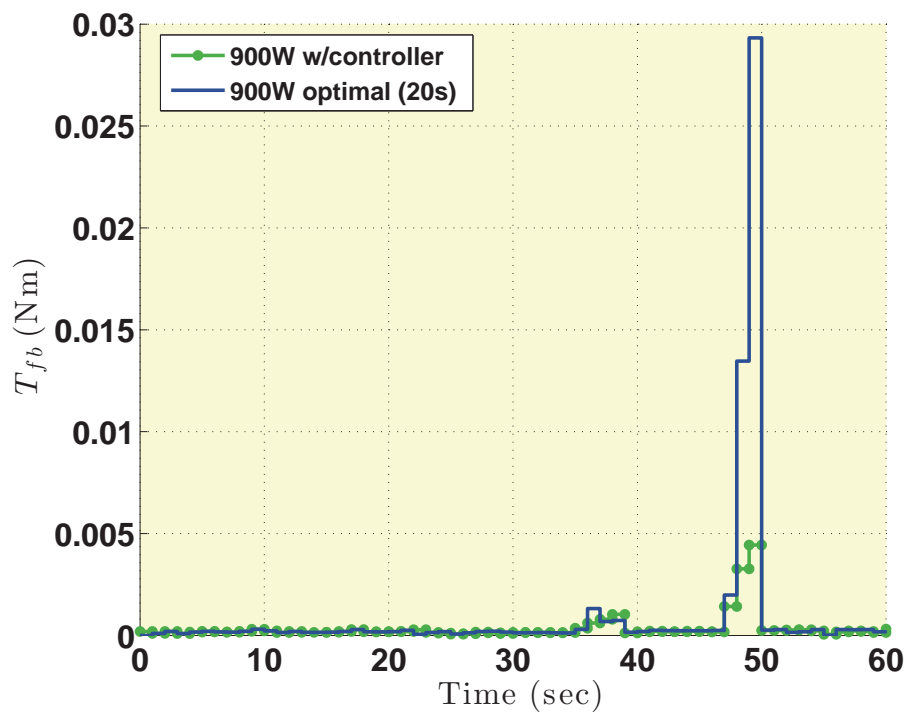

Figure D.1: Brake force torque trajectories 


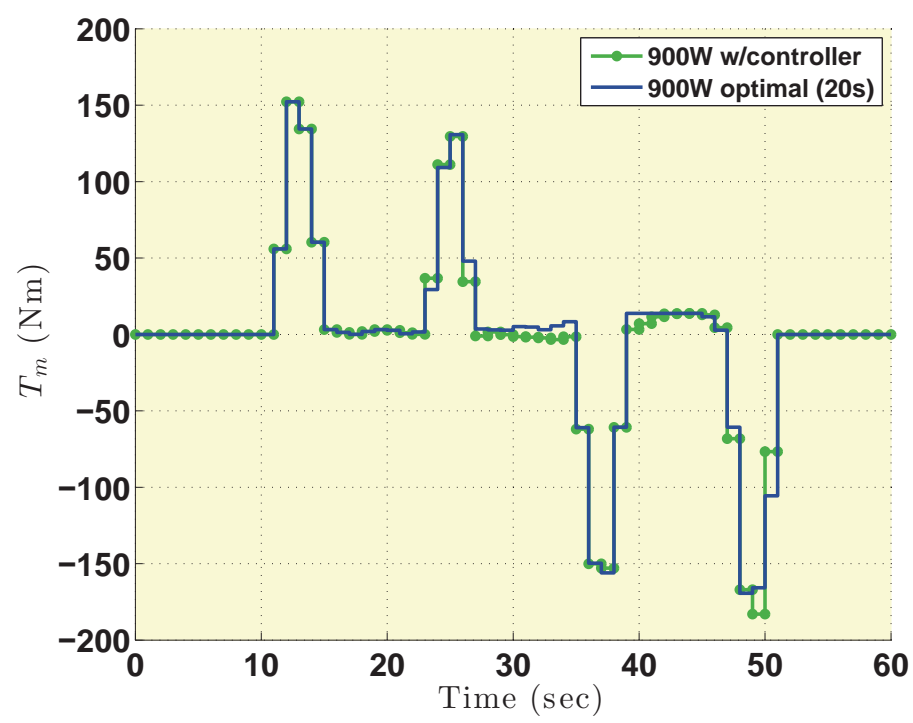

Figure D.2: Motor torque trajectory

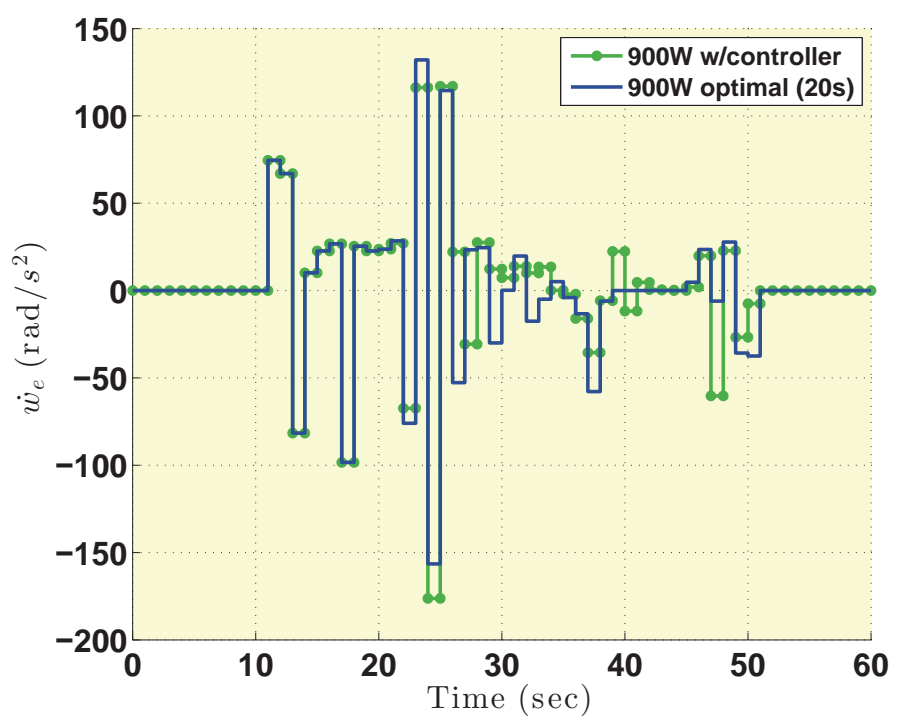

Figure D.3: Engine speed acceleration 


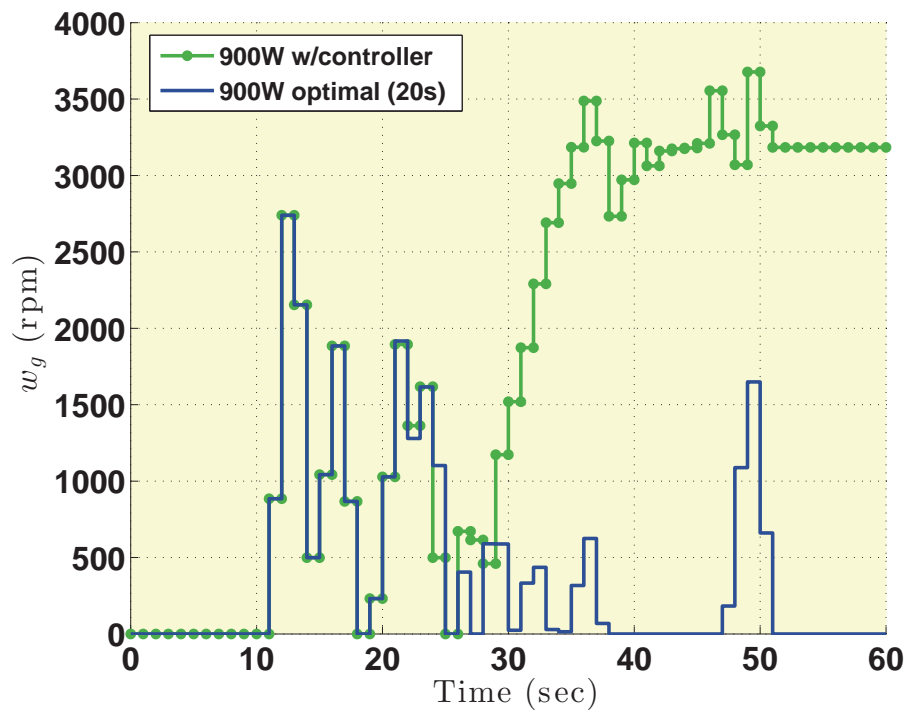

Figure D.4: Generator speed

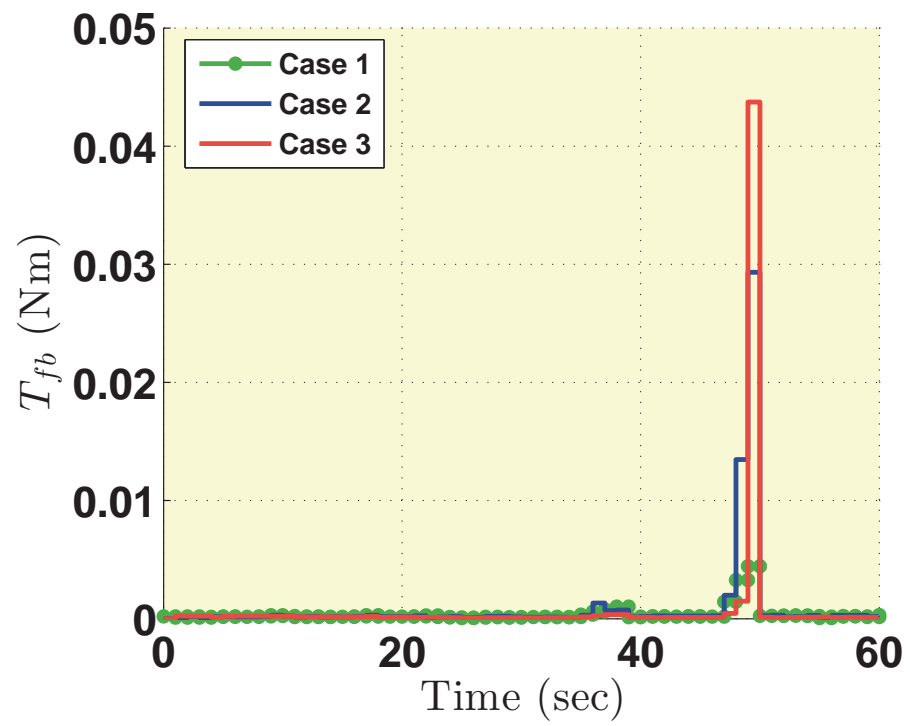

Figure D.5: Brake force torque trajectory for all three cases 


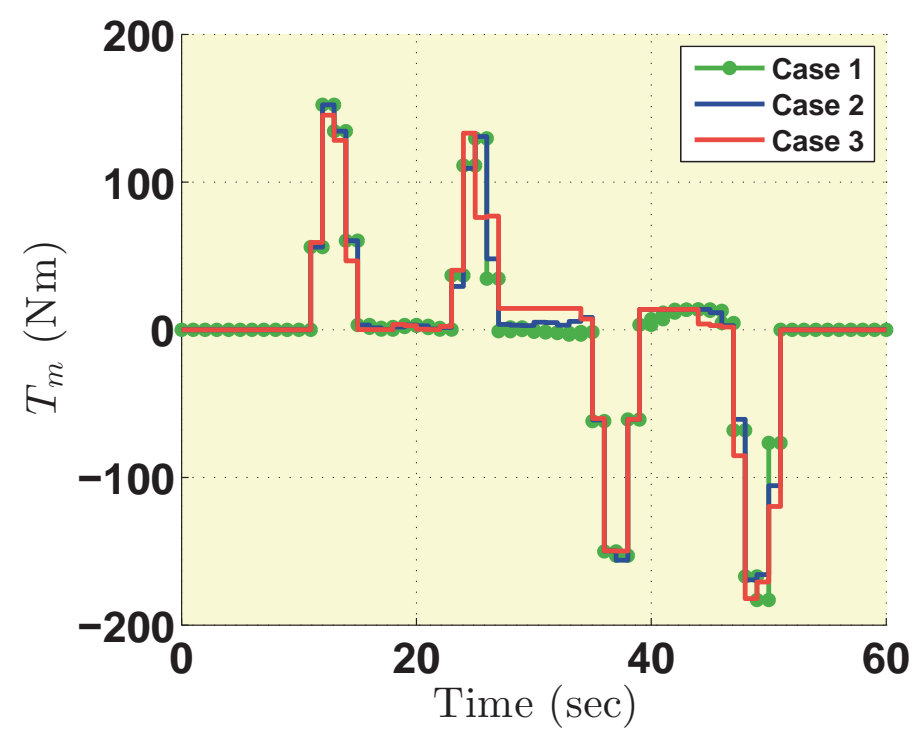

Figure D.6: Motor torque trajectory for all three cases

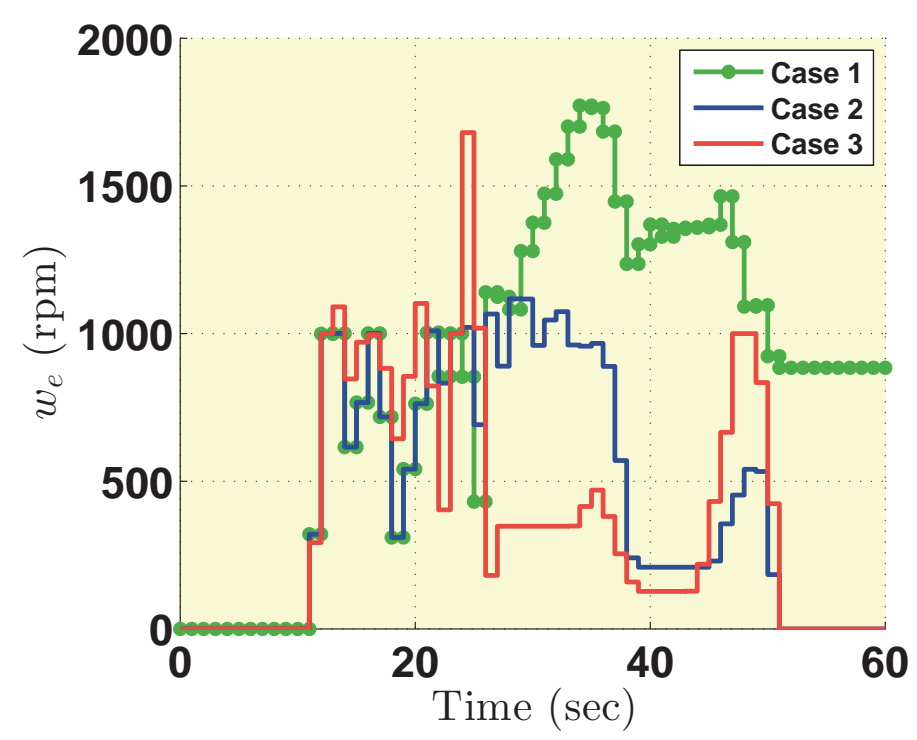

Figure D.7: Engine speed for all three cases 


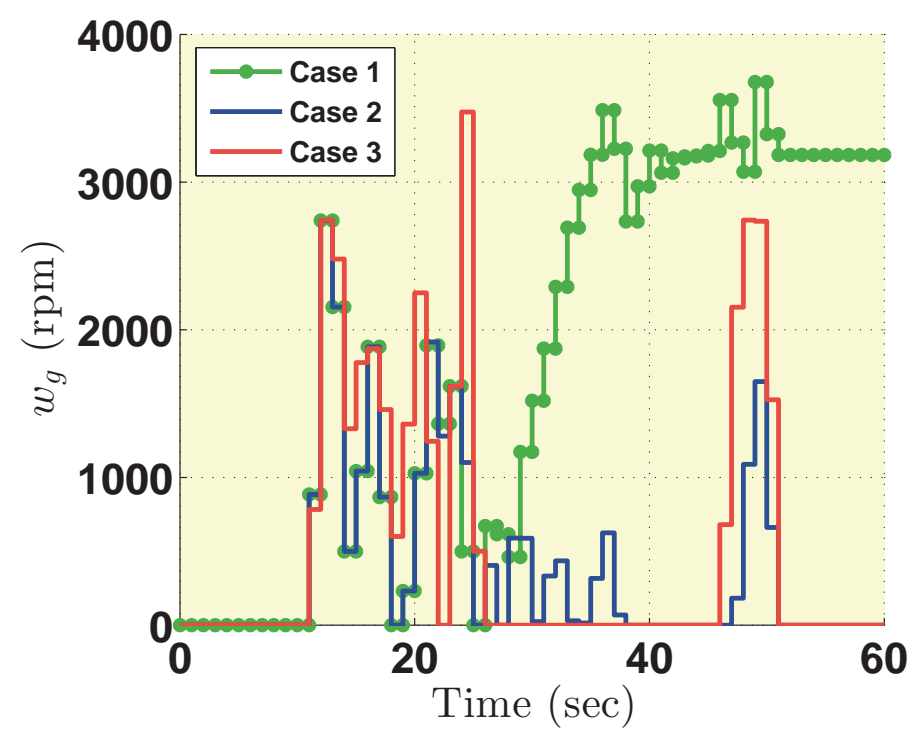

Figure D.8: Generator speed for all three cases

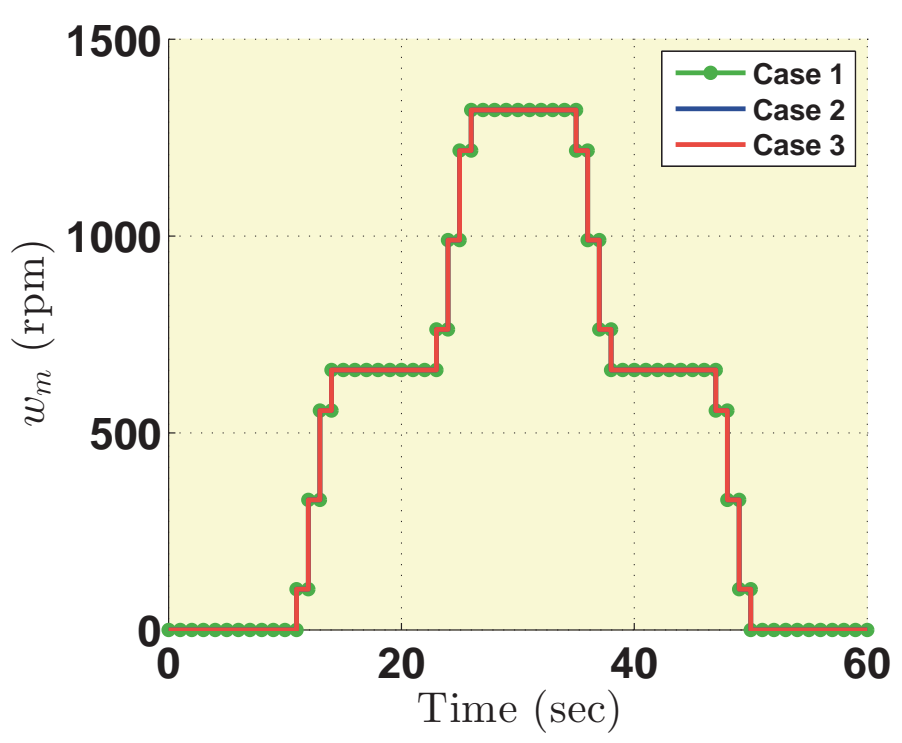

Figure D.9: Motor speed for all three cases 



\section{Appendix E}

\section{Supporting Figures for Design \\ Optimization}

The following figures support the design optimization results. 


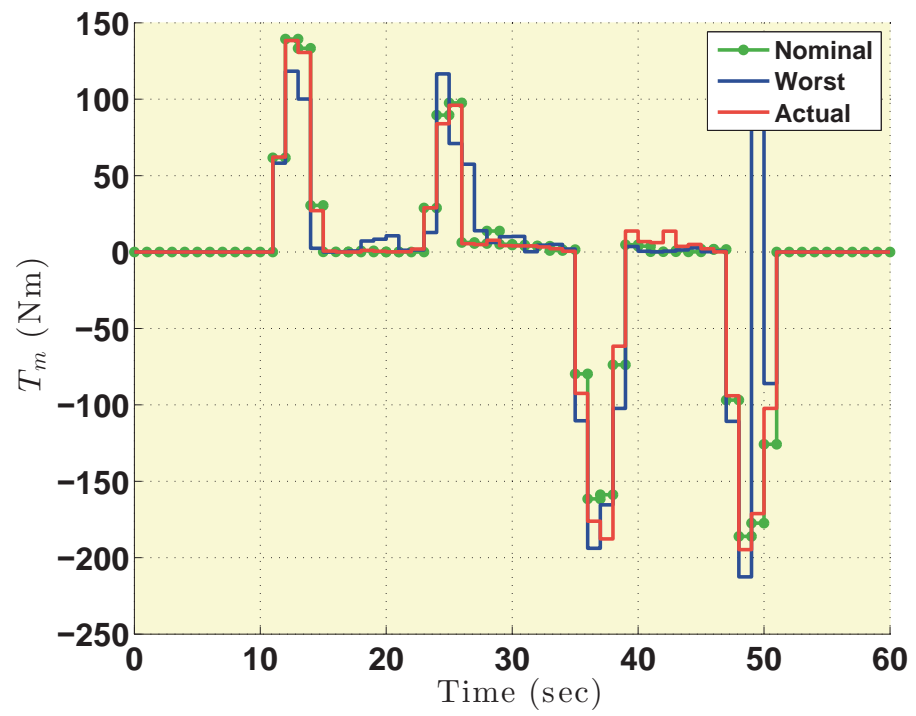

Figure E.1: Motor torque trajectories for different electrical duty cycles

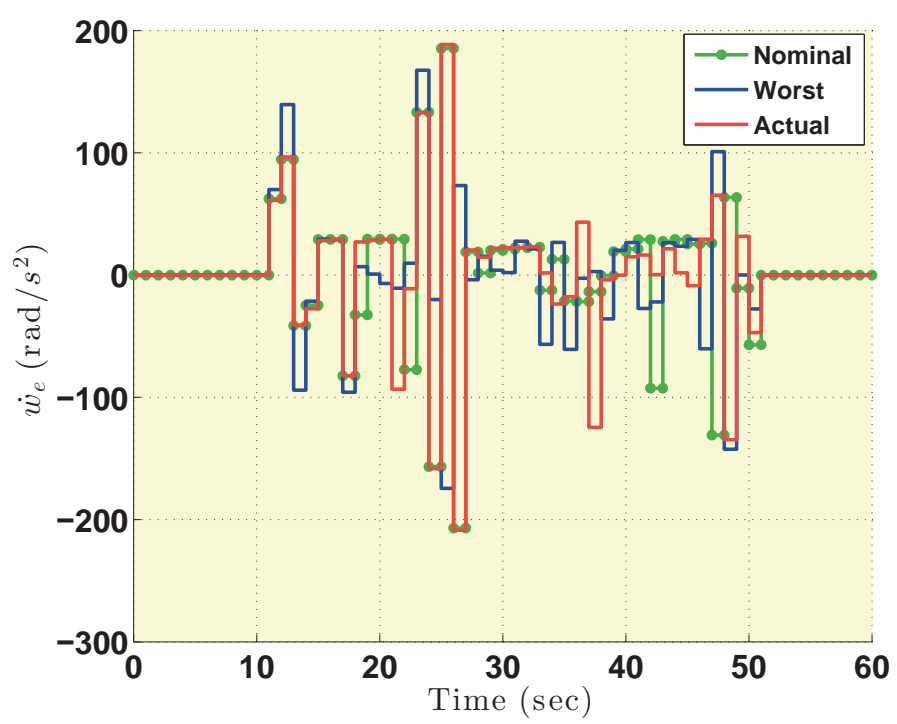

Figure E.2: Engine speed acceleration for different electrical duty cycles 


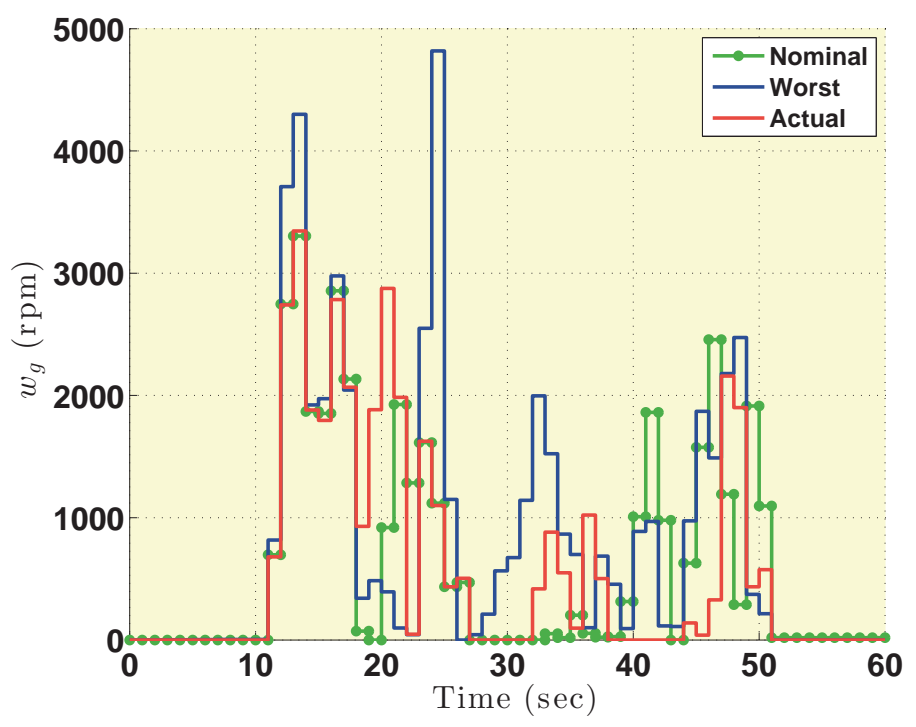

Figure E.3: Generator speed for different electrical duty cycles 



\section{Appendix F}

\section{Letters of Permission}

Letter of permission to use copyrighted materials for Chapters 1 and 5 .

From: Denise Rizzo [mailto:dmrizzo@mtu.edu] Sent: 20 October 2014 12:17 To: M Dorgham Subject: Using papers for my dissertation

Dear Editor:

I would like to use these two papers for my $\mathrm{PhD}$ dissertation:

Kramer, D.M. and Parker, G.G. (2011) Current state of military hybrid vehicle development, Int. J. Electric and Hybrid Vehicles, Vol. 3, No. 4, pp.369-387.

Rizzo, D.M. and Parker, G.G. (2014) Determining optimal state of charge for a military vehicle microgrid, Int. J. Powertrains, Vol. 3, No. 3, pp.303-318.

Is this ok? Is there any paper work that needs to be filled out? 
Thank you, Denise Rizzo

Jeanette Brooks ijrb@inderscience.com; Oct 20

to me Dear Denise,

Thank you for your email. Inderscience is happy to give you the permission to use these papers in your $\mathrm{PhD}$ dissertation, provided that full acknowledgement of the original source of publication is made clear, and a statement included that Inderscience retains copyright of the articles.

Kind regards and good luck!

Jeanette

J R Brooks (Dr) Publications Director Email: jrb@inderscience.com

Inderscience Enterprises Limited World Trade Centre Building II 29 route de Pre-Bois Case Postale 856 CH-1215 Geneve 15 Switzerland

cid:89D9FD8E-5C90-442B-A2BE-8B18CC28CAFD@lan

Sign up to our Newsletter for highlights from our journals and related industry events: http://www.inderscience.com/newsletter 